\title{
Comparative Effectiveness of 12 Treatment Strategies for Preventing Contrast- Induced Acute Kidney Injury: A Systematic Review and Bayesian Network
}

\section{Meta-analysis}

Xiaole Su, MD, ${ }^{1,2^{*}}$ Xinfang Xie, MD, ${ }^{1 *}$ Lijun Liu, MD, ${ }^{1}$ Jicheng Lv, MD, ${ }^{1}$ Fujian Song, $\mathrm{PhD},{ }^{3}$ Vlado Perkovic, MD, ${ }^{4}$ Hong Zhang, $\mathrm{MD}^{1}$

* Both authors contributed equally

${ }^{1}$ Renal Division, Peking University First Hospital; Peking University Institute of Nephrology; Key Laboratory of Renal Disease, Ministry of Health of China; Key Laboratory of Chronic Kidney Disease Prevention and Treatment (Peking University), Ministry of Education, No.8, Xishiku Street, Xicheng District, Beijing, China,;

${ }^{2}$ Renal Division, Shanxi Medical University Second Hospital, Shanxi Kidney Disease Institute, No.382, Wuyi Road, Xinghualing Distirct, Taiyuan, China;

${ }^{3}$ Department of Population Health \& Primary Care, Norwich Medical School, Faculty of Medicine and Health Science, University of East Anglia, Norwich, Norfolk, United Kingdom.

${ }^{4}$ The George Institute for Global Health, University of Sydney, Sydney, New South Wales, Australia.

Running title: Optimal Strategies in Preventing Contrast-Induced Acute Kidney Injury

Corresponding Author: Lijun Liu

Renal Division, Peking University First Hospital

Institute of Nephrology, Peking University

No. 8, Xishiku Street, Xicheng District, Beijing, China. 100034

Telephone: 86-10-83572388

Fax: 86-10-66551055

E-mail: lijun.liu@medmail.com.cn

Word count of abstract: 312

Word count of text: 3270

Table: 0

Figures: 4 


\begin{abstract}
Background: To simultaneously evaluate the relative efficacy of multiple pharmacological strategies for preventing contrast-induced acute kidney injury (CIAKI).
\end{abstract}

Study Design: Systematic review containing a Bayesian network meta-analysis of randomized controlled trials (RCTs)

Setting \& Population: Participants undergoing diagnostic and/or interventional procedures with contrast media.

Selection Criteria for Studies: RCTs comparing the active drug treatments with each other or with hydration alone.

Intervention: Any of the following drugs in combination with hydration: Nacetylcysteine (NAC), theophylline (aminophylline), fenoldopam, iloprost, alprostadil, prostaglandin E1, statins, statins plus NAC, bicarbonate sodium, bicarbonate sodium plus NAC, ascorbic acid (vitamin C), tocopherol (vitamin E), alpha lipoic acid, atrial natriuretic peptide, B-type natriuretic peptide, and carperitide.

Outcomes: The occurrence of CI-AKI

Results: The trial network included 150 trials with 31,631 participants and 4,182 CIAKI events assessing 12 different interventions. Compared with hydration, the odds ratios (ORs) for CI-AKI were 0.31 (95\% credible interval 0.14 to 0.60 ) for high-dose statin plus NAC, 0.37 (0.19 to 0.64$)$ for high-dose statins alone, 0.37 ( 0.17 to 0.72 ) for prostaglandins, 0.48 (0.26 to 0.82$)$ for theophylline, 0.62 (0.40 to 0.88$)$ for bicarbonate sodium plus NAC, 0.67 (0.54 to 0.81 ) for NAC alone, 0.64 (0.41 to 0.95 ) 
for vitamins and its analogues, 0.70 (0.29 to 1.37) for natriuretic peptides, 0.69 (0.31 to 1.37) for fenoldopam, 0.78 (0.59 to 1.01 ) for bicarbonate sodium, and 0.98 (0.41 to 2.07) for low dose statin. High-dose statin plus NAC or high-dose statin alone were likely to be ranked the best or the second best for preventing CI-AKI. The overall results were not materially changed in meta-regressions, subgroup and sensitivity analyses.

Limitations: Patient-level data was unavailable. Unable to include some treatment agents, low event rates, and imbalanced distribution of participants among treatment strategies.

Conclusions: High-dose statins plus hydration with or without NAC might be the preferred treatment strategy to prevent CI-AKI in patients undergoing diagnostic and/or interventional procedures requiring contrast media.

Index Words:

contrast-induced acute kidney injury (CI-AKI), contrast media, kidney disease, acute kidney failure, statins, hydroxymethylglutaryl-CoA reductaseinhibitor, atorvastatin, rosuvastatin, simvastatin, serum creatinine, cardiovascular events, systematic review. 


\section{Introduction}

With the steady increase in the rates of diagnostic and/or interventional procedures with contrast media (CM), contrast-induced acute kidney injury (CI-AKI) has become the third most common cause of AKI in hospitalized patients. ${ }^{1} \mathrm{CI}-\mathrm{AKI}$ leads to prolonged hospitalization, increased costs, and increased morbidity and mortality. ${ }^{2}$ Factors associated with the risk of CI-AKI include pre-existing renal functional impairment, diabetes, hypertension, chronic heart failure, advanced age, volume depletion, haemodynamic instability, use of concurrent nephrotoxic medications, and large volume or high osmolality of CM. ${ }^{3,4}$

Minimization of the CM dose and the use of iso-osmolar or low-osmolar CM are recommended as non-pharmacological precautions, and numerous pharmacological strategies for preventing CI-AKI have been evaluated. In 2008, a comprehensive meta-analysis of randomized controlled trials (RCTs) concluded that $N$ acetylcysteine (NAC) in combination with hydration was more effective than hydration alone. ${ }^{5}$

However, due to the lack of head-to-head comparisons between treatment agents, traditional pairwise meta-analyses could not be used to simultaneously synthesize all evidence and generate clear hierarchies for the efficacy of different treatments..$^{5-8}$ Therefore, the choice of the best treatment in practice is generally based on subjective judgement, and objective information regarding the relative efficacy of different interventions would help the development of clinical practice guidelines for preventing CI-AKI.

Bayesian network meta-analysis, also known as mixed treatment comparison, 
enables indirect comparison using a common comparator, and combines direct and indirect comparisons to synchronously assess multiple treatments. ${ }^{9-11}$ The usefulness of this method has been demonstrated in many studies on various medical conditions and interventions. ${ }^{12-14}$ This systematic review and network meta-analysis therefore aims to compare the relative efficacy of different pharmacological interventions for preventing CI-AKI by means of network meta-analysis within a Bayesian framework.

\section{Methods}

\section{Data Sources and Searches}

This systematic review was performed according to a pre-specified protocol (Item S1) and the reporting was in line with PRISMA guidelines. ${ }^{15}$ We searched MEDLINE via Ovid (from 1946 to May 2016), Embase (from 1966 to May 2016), and the Cochrane Library database (Cochrane Central Register of Controlled Trials; before May 2016) for RCTs of CI-AKI prevention, without any language restrictions (see Item S1 for full search terms). The ClinicalTrials.gov website was also searched for RCTs that were registered as completed but not yet published.

\section{Study Selection}

We included RCTs that evaluated any of the following drugs in combination with hydration: NAC, theophylline (aminophylline), fenoldopam, iloprost, alprostadil, prostaglandin E1, statins, statins plus NAC, bicarbonate sodium, bicarbonate sodium plus NAC, ascorbic acid (vitamin C), vitamin E or its analogues (tocopherol), alpha 
lipoic acid, atrial natriuretic peptide, B-type natriuretic peptide, and carperitide. RCTs comparing the above active drug treatments with each other or with hydration were eligible. We excluded studies that contained only one or none of the above treatments. Eligible participants were those who underwent diagnostic and/or interventional procedures with $\mathrm{CM}$, such as diagnostic coronary or peripheral arterial angiography or percutaneous intervention, ventriculography, enhanced CT, intravenouspyelography, and other relevant procedures.

The treatment groups were classified into 12 categories according to the drug species and/or dose: 1. atrial natriuretic peptide, B-type natriuretic peptide and carperitide were classified into natriuretic peptide; 2. ascorbic acid (vitamin C, tocopherol and alpha-lipoic acid were classified into vitamins and its analogues; 3. simvastatin 40-80 mg, rosuvastatin 20-40 mg and atorvastatin 40-80 mg were known as high-dose statin; 4. low-dose statin included simvastatin 10-20 mg, rosuvastatin 10 mg and atorvastatin 10-20 mg; 5. iloprost, alprostadil, misoprostol and prostaglandin E1 were categorized into prostaglandin. The other seven treatments included: 6 . theophylline (aminophylline); 7. NAC; 8. fenoldopam; 9. bicarbonate sodium; 10. bicarbonate sodium plus NAC; 11. high-dose statin plus NAC; 12 . hydration

\section{Data Extraction and Quality Assessment}

Study selection, data extraction, and quality assessment were performed independently by two investigators (XL.S and XF.X) according to the pre-specified study protocol (Item S1). The two investigators screened the titles and abstracts of the records identified by the search strategies for eligibility. Disagreements were resolved 
by discussion with a third reviewer (LJ.L). Data on pre-specified variables from the included studies were extracted into a computerized spreadsheet.

The outcome used was the development of CI-AKI, defined as an absolute increase in the baseline serum creatinine level of greater than $44.2 \mu \mathrm{mol} / \mathrm{L}(0.5 \mathrm{mg} / \mathrm{dL})$ or a relative increase of greater than $25 \%$, typically within $48-72 \mathrm{~h}$ after contrast injection. ${ }^{16,17}$ If data was not available for the first $48-72 \mathrm{~h}$ after the treatment, we used data obtained within the first 5 days of treatment (the data point closest to 48-72 $\mathrm{h}$ was given preference). ${ }^{18}$ If different measurement index (eg. eGFR, Ccr) or standard was applied, we extracted data according to one defined by authors of the included studies.

We assessed sources of bias using the Cochrane Collaboration risk-of-bias tool, ${ }^{19}$ including an assessment of financial conflicts of interest. ${ }^{20}$ We developed operational definitions for high, low, and unclear risk of bias for each of the eight validity domains (Item S2).

\section{Data Synthesis and Analysis}

We used odds ratio (OR) and its 95\% credible intervals (CrIs) to measure the relative effect of different treatments on CI-AKI outcome. Before conducting network meta-analysis, we conducted conventional pairwise meta-analyses for treatments that were directly compared in RCTs. We used fully Bayesian method (FB), assuming a binomial likelihood on the log-odds scale, in pairwise meta-analyses through WinBUGS 1.4.3..$^{21,22}$ To investigate heterogeneity in conventional pairwise metaanalysis, we used STATA 12.0 to conduct meta-regression of direct comparisons 
based on empirical Bayes method, and estimated $I^{2}, t a u^{2}$ and $Q$ value.

Network meta-analysis was conducted by using random-effects model within a Bayesian framework, assuming a binomial likelihood and using WinBUGS 1.4 .3 and R2WinBUGS package of R software 3.1.1 according to a pre-defined protocol (Item S1). We used non-informative priors with vague normal (mean, 0; variance, 100,000) and uniform (0 to 5) prior distributions for parameters such as means and standard deviations, respectively. ${ }^{11}$ For each analysis, we generated 200,000 simulations for each of the two sets of different initial values, and discarded the first 80,000 simulations as the burn-in period. Convergence was reached when Rhat, the potential scale reduction factor is close to 1 for each of the parameters using the BrooksGelman-Rubin statistic. ${ }^{23}$ We used the surface under the cumulative ranking (SUCRA) probabilities to rank the treatments. ${ }^{24}$

Inconsistency refers to differences in effect estimates between direct and indirect comparisons, which could be assessed when three treatments are connected within a loop. ${ }^{25,26}$ For each closed loop, we estimated the absolute difference between the direct and indirect comparisons, which is termed inconsistency factor. Inconsistent loops were identified by a significant disagreement (inconsistency factor and its $95 \%$ CI that excludes 0 ) between direct and indirect evidence. ${ }^{25,27,28}$ As a whole, inconsistency was also assessed by the comparison between the consistency model and inconsistency model of the network meta-analysis using deviance information criterion (DIC). Alower value of the DIC suggests a more parsimonious model. If the trade-off between model fit and complexity favours the model with assumes 
inconsistency, then the assumption of consistency is likely to be violated. ${ }^{12,29}$

We carried out the following pre-specified sensitivity analyses: exclusion of trials with sample sizes less than 50 in order to reduce small study effect and publication bias; exclusion of trials with high-osmolar and unspecified CM types; and exclusion of data from patients with non-DM (Item S1). Other analyses were post-hoc: exclusion of trials evaluating only patients with normal kidney function, published before 2004, with oral hydration and unspecified hydration agent.

Pre-specified multiple-treatments meta-regression and subgroup analyses were conducted by several major covariates, such as mean CM dose, mean age, baseline serum creatinine concentration, different CI-AKI definitions, and different radiologic procedures with CM (Item S1). Post-hoc subgroup analysis was conducted by types of CM and different hydration agents.

The models used, the WinBUGS codes, and R routines for all results are presented in detail and exemplified in http://www.mtm.uoi.gr. and http://www.nicedsu.org.uk. A short summary is supplied in Item S3.

\section{Results}

The literature search yielded 4144 articles. We assessed the full text of 396 of these articles for eligibility, and eventually included 150 RCTs in the network meta-analysis

(Figure 1, see details of included studies in Table S1). CI-AKI was measured according to the difference between the baseline serum creatinine level and the level within $48 \mathrm{~h}-72 \mathrm{~h}$ in 120 studies. In 30 trials, CI-AKI was defined according to different 
points in time and measurements (eg. eGFR, Ccr), or the determination method was not specified. Of the included RCTs, 104 trials included patients with impaired renal function, 37 trials included patients with normal or impaired renal function, and 9 trials included only patients with normal renal function. Participants were recruited at an average age of 67 years, and male participants accounted for $68 \%$ of the total population. Atotal of 11 types of CM were used, including iso-osmolar, lowosmolar, and high-osmolar media. The dosing regimens and types of CM used in the included trials are detailed in Table S1.

The methodological quality of the included trials was not high overall and varied substantially (Item S2, Figure S1, S2). The proportion of trials with a low risk of bias was $53 \%$ in terms of random sequence generation, $54 \%$ in terms of allocation concealment, $49 \%$ in terms of blinding of both participants and health care professionals, 59\% in terms of blinding of outcome assessors, $48 \%$ in terms of attrition, and 35\% in terms of reporting bias. With respect to conflicts of interest, about $50 \%$ of RCTs were funded by pharmaceutical industry and $51 \%$ reported author-industry financial relationships. In order to investigate reporting/published bias, we searched and found 21 protocols for 396 full-text reviewed articles. In studies without reporting the outcome of interest, we didn't find any pre-planned CI-AKI outcome.

A total of 4,182 CI-AKI events were reported in 150 trials with 31,631 participants. Figure 2 shows all comparisons that were analysed in the network meta-analysis. The results of available direct comparisons are shown in Figure $\mathbf{3}$ and Table S2, and the 
results of testing heterogeneity $\left(I^{2}, \operatorname{tau}^{2}\right.$ and $\left.Q\right)$ within treatment strategies were showed in Table S2. We summarized the results of random-effects consistency network meta-analysis in Figure 3. The effects of individual treatment strategies compared with hydration on preventing CI-AKI are presented in Figure 4. Compared with hydration alone, high-dose statin plus NAC, high-dose statin, prostaglandin, theophylline, bicarbonate sodium plus NAC, vitamins and their analogues and NAC alone (all in combination with hydration) statistically significantly reduced the risk of CI-AKI (Figure 3 and Figure 4). In addition, high-dose statins were significantly more effective than low-dose statins (OR: 0.42; 95\% CrI: 0.19 to 0.79), NAC (OR: 0.51; 95\% CrI: 0.29 to 0.98 ) and bicarbonate sodium(OR: 0.49 ; $95 \%$ CrI: 0.23 to 0.86). Prostaglandin was significantly more effective than bicarbonate sodium (OR: 0.49; $95 \%$ CrI: 0.22 to 0.98 ). High-dose statin combined with NAC was statistically more effective than NAC (OR: 0.41; 95\% CrI: 0.22 to 0.86), low-dose statins (OR: 0.28; 95\% CrI: 0.12 to 0.99), bicarbonate sodium plus NAC (OR: 0.51; 95\% CrI: 0.24 to 0.99 ) and bicarbonate sodium alone (OR: 0.35 ; $95 \%$ CrI: 0.18 to 0.79 ).

High-dose statin plus NAC (SUCRA: 0.90) and high-dose statin (SUCRA: 0.83) were most likely to be ranked the best or second best (Figure S3). They were followed by prostaglandins (SUCRA: 0.82) and theophyllines (SUCRA: 0.70). SUCRAs (range: 0.41 to 0.49 ) and rankings were similar for bicarbonate sodium plus NAC, vitamins and its analogues, natriuretic peptides, fenoldopam and NAC. Hydration alone was ranked as the least effective treatment. The DIC value was lowest in the random consistency model than in the other three 
models, which indicated that the former was the preferred model with a better tradeoff between model fit and complexity (Table S3). However, significant discrepancy between the direct and indirect comparisons was identified in two of the 13 loops (Figure S4). The two inconsistent loops consisted of (1) vitamins and its analogues vs. bicarbonate sodium plus NAC vs. hydration; and (2) vitamins and its analogues vs. bicarbonate sodium plus NAC vs. NAC. Further investigation was performed, but we were unable to identify possible sources of inconsistency. As the number of relevant studies in the inconsistent loops was small, the extent of inconsistency was not substantial enough to affect the overall results.

Treatments with high-dose statin plus NAC or high-dose statin were consistently associated with the lowest or second lowest incidence of CI-AKI in sensitivity analyses, meta-regression, and subgroup analyses (Table S4 and S5). A notable exception is that the effects of vitamins and its analogues became statistically nonsignificant compared with hydration in some sensitivity and subgroup analyses.. When the analysis included only DM participants (38 trials, 7984 patients and 826 events), credible intervals were wide and all ORs were no longer statistically significant due to the reduced sample size, although high-dose statin plus NAC and high-dose statin remained the first and the second ranking among all treatment strategies. None of the other sensitivity, meta-regression, or subgroup analyses led to important changes in the overall results (Table S4 and S5).

\section{Discussion}


Our study included 150 trials with more than 30,000 participants and a total of 4,182 CI-AKI events. The mixed treatment comparison of 12 treatment strategies for preventing CI-AKI confirmed that treatment with a high-dose statin alone or in combination with NAC (both in combination with hydration) during CM administration significantly reduced the risk of CI-AKI compared with hydration alone. Compared with other protective regimens, oral administration of high-dose statins is simple and convenient. These results indicate an opportunity to potentially simplify prevention strategies for CI-AKI. Our analysis also found a number of other strategies that appeared to be superior to hydration alone, including prostaglandins, theophylline, bicarbonate sodium plus NAC, vitamins and their analogues and NAC.

A recent comprehensive pairwise meta-analysis reported that the greatest reduction in CI-AKI was seen with NAC plus hydration and with statins plus NAC plus hydration in patients receiving $\mathrm{CM} .{ }^{30}$ Our study simultaneously compared multiple treatment strategies using Bayesian network meta-analysis method, and found that patients using CM were most likely to benefit from high-dose statin. The results of the current study were similar to findings from previous primary studies ${ }^{31-34}$ and metaanalyses, ${ }^{6,35-37}$ which suggested that short-term prophylaxis with high-dose statins led to a significant reduction in the risk of CI-AKI. In contrast, the meta-analysis by Zhang $\mathrm{T}$ et al. ${ }^{38}$ found no significant reduction in the incidence of CI-AKI with statins treatment, as determined using the pooled estimate of the included trials. However, it should be noted that the meta-analysis by Zhang $\mathrm{T}$ et al incompletely include relevant randomized trials. ${ }^{39,40}$ 


\section{Pre-existing renal dysfunction is an independent predictor of CI-AKI. ${ }^{41}$ Findings} from some previous pairwise meta-analyses ${ }^{35,36}$ suggested that the use of statins may not be effective for patients with pre-existing chronic kidney disease (CKD). Our meta-regression analyses found that baseline serum creatinine concentration as a continuous covariate was not a statistically significant effect-modifier (regression coefficient: 0.09; 95\% CrI: -1.86 to 1.90$)$. Consistent with our results, the TRACK-D study involving almost 3,000 DM participants with mild-to-moderate CKD demonstrated a significant reduction in the relative risk of CI-AKI with rosuvastatin therapy. ${ }^{40}$

Although the pathogenesis of CI-AKI is not completely understood, multiple mechanisms are probably involved, including direct toxicity of CM on the renal tubular epithelium, inflammatory reactions, oxidative stress, ischemic injury, and renal tubular obstruction. ${ }^{42}$ Statins may have multiple non-lipid-lowering effects, such as enhancement of endothelial nitric oxide production, ${ }^{43-45}$ anti-inflammatory and anti-oxidative actions, ${ }^{46,47}$ and apoptosis prevention. ${ }^{34}$ These pleiotropic effects of statins could mediate the reduction of CI-AKI risk after iodinated contrast administration. Furthermore, use of antioxidants (eg, vitamins and its analogues, NAC) might be an effective strategy to prevent CI-AKI, considering their roles in attenuating the oxidative damage from radiocontrast.

Apart from high-dose statins and high-dose statins plus NAC, prostaglandins had better effects than other treatment strategies, based on evidence from five trials that included a total of 943 participants and evaluated four classes of prostaglandins. 
Adequate renal prostaglandin levels may counteract contrast-induced renal vasoconstriction and selective renal tubular epithelial cell toxicity. ${ }^{48}$

As a non-selective adenosine receptor antagonist, theophylline may help attenuate the vasoconstrictive tendencies observed after $\mathrm{CM}$ administration. ${ }^{49,50} \mathrm{~A}$ previous pairwise meta-analysis also showed that theophylline administration reduced the incidence of CI-AKI compared with the control group. ${ }^{51}$ Although pre-interventional theophylline administration might be helpful in patients with CM, the possibility of cardiovascular side-effects and the interactions with numerous drugs associated with theophylline should be recognized..$^{52,53}$

Many but not all studies reported that NAC has a protective effect on CI-AKI when administered before the onset of renal insult. Of the 11 previous meta-analyses published on this subject, seven found a net benefit of NAC in CI-AKI prevention. However, due to statistically significant heterogeneity and possible publication bias, the benefit of NAC might have been overestimated. ${ }^{54}$ With low the strength of evidence, Kidney Disease Improving Global Outcomes Clinical Practice Guideline for CI-AKI suggests the use of oral $N$-acetylcysteine plus hydration. Another metaanalysis found that a combination of NAC and sodium bicarbonate substantially reduced CI-AKI risk compared with NAC alone. ${ }^{55}$ However, we found that both sodium bicarbonate plus NAC and vitamins and their analogues were involved in significant inconsistent loops, and results for vitamins and their analogues were not robust in sensitivity and subgroup analyses.

This network meta-analysis provides a most comprehensive picture of the 
likelihood of a range of treatments to prevent CI-AKI, and reports the results of mixed comparisons of multiple treatments that have been rarely compared in head to head trials. We also report the ranking probability for all 12 treatment agents. However, treatment rankings derived from network meta-analyses may have a substantial degree of imprecision, ${ }^{56}$ and the results in terms of treatment ranking should beinterpreted with caution.

Our study has several limitations. First, the trial network could not include some treatment agents, such as $\mathrm{Na} / \mathrm{K}$ citrate, ${ }^{57}$ allopurinol, ${ }^{58}$ statin plus alsprostadil, ${ }^{59}$ that may be efficacious but were evaluated in only one or two small trials without a connection with other commonly used treatments. For many specific treatments, the number of patients and events in the available trials may not be sufficient to form a well-connected network for meta-analysis. We therefore combined drugs with the same types and similar mechanism of action and evaluated treatment effects of major drug classes. Second, many of the included studies showed low CI-AKI event rates or no events at all in one or both trial arms, and there was an imbalance in the distribution of participants among some of the treatment strategies. Consequently, the uncertainty in the analyses was increased, resulting in wide CrIs for several treatment comparisons. Third, the absence of patient-specific data, varying quality and design of the included studies are limitations common to all meta-analyses. To at least partly nullify the latter factors, we included only RCTs. Furthermore, the meta-regression based on trial-level covariates rather than individual patient data might bring the ecological fallacy. ${ }^{60}$ Fourth, we included only published studies in this analysis, and 
reporting bias could not be ruled out because not all studies reported CI-AKI outcome, especially when CI-AKI events were not primary end points.

Further studies with head-to-head comparisons of statins at both high and low doses were needed to illuminate whether important differences exist in their abilities to reduce CI-AKI risk and whether dose matters. Prospective randomized trials should focus on relatively homogeneous patient populations, such as DM, or whether patients with different stages of CKD would benefit similarly or differently from periprocedural statin therapy. Future studies are also needed to test effects of combinations of different strategies shown to be beneficial in this analysis, and to uncover possible mechanisms.

Our Bayesian network meta-analysis indicates the effects and superiority of using a high-dose statin plus hydration with or without NAC in patients undergoing diagnostic and/or interventional procedures requiring CM. The results should be interpreted with caution due to important data and methodological limitations.

\section{ACKNOWLEDGEMENTS}

Support: This work was supported by grants from the National Natural Science Foundation (no. 81100503) and the Natural Science Foundation of China to the Innovative Research Group (no. 81021004). Study sponsors had no role in study design; collection, analysis, and interpretation of data; writing the report; and the decision to submit the report for publication.

Financial Disclosure: The authors declare that they have no other relevant financial 
interests.

Contributions: Research idea and study design: LL, XS, XX, JL, HZ; data acquisition: XS, XX; data analysis/interpretation: LL, XS, XX, VP, FS; statistical analysis: LL, XS, XX, FS; supervision or mentorship: LL, JL, VP, HZ. Each author contributed important intellectual content during manuscript drafting or revision and accepts accountability for the overall work by ensuring that questions pertaining to the accuracy or integrity of any portion of the work are appropriately investigated and resolved. LL take responsibility that this study has been reported honestly, accurately, and transparently; that no important aspects of the study have been omitted; and that any discrepancies from the study as planned have been explained.

\section{References}

1. Nash K, Hafeez A, Hou S. Hospital-acquired renal insufficiency. Am J Kidney Dis. 2002; 39(5): 930-936.

2. McCullough PA. Contrast-induced acute kidney injury. J Am Coll Cardiol. 2008; 51(15): 1419-1428.

3. Mehran R, Nikolsky E. Contrast-induced nephropathy: definition, epidemiology, and patients at risk. Kidney Int Suppl. 2006; (100): S11-15.

4. McCullough PA, Adam A, Becker CR, et al. Risk prediction of contrast-induced nephropathy. Am J Cardiol. 2006; 98(6a):27k-36k. 
5. Kelly AM, Dwamena B, Cronin P, Bernstein SJ, Carlos RC. Meta-analysis: effectiveness of drugs for preventing contrast-induced nephropathy. Ann Intern Med. 2008; 148(4): 284-294.

6. Ukaigwe A, Karmacharya P, Mahmood M, et al. Meta-analysis on efficacy of statins for prevention of contrast-induced acute kidney injury in patients undergoing coronary angiography. Am J Cardiol. 2014; 114(9): 1295-1302.

7. Jang JS, Jin HY, Seo JS, et al. Sodium bicarbonate therapy for the prevention of contrast-induced acute kidney injury - a systematic review and meta-analysis. Circ $J$. 2012; 76(9): 2255-2265.

8. Sadat U, Usman A, Gillard JH, Boyle JR. Does ascorbic acid protect against contrast-induced acute kidney injury in patients undergoing coronary angiography: a systematic review with meta-analysis of randomized, controlled trials. J Am Coll Cardiol. 2013; 62(23): 2167-2175.

9. Ades AE, Sculpher M, Sutton A, et al. Bayesian methods for evidence synthesis in cost-effectiveness analysis. Pharmacoeconomics.2006; 24(1): 1-19.

10. Song F, Altman DG, Glenny AM, Deeks JJ. Validity of indirect comparison for estimating efficacy of competing interventions: empirical evidence from published meta-analyses. BMJ. 2003; 326(7387): 472.

11. Lu G, Ades AE. Combination of direct and indirect evidence in mixed treatment comparisons. Stat Med. 2004; 23(20): 3105-3124.

12. Leucht S, Cipriani A, Spineli L, et al. Comparative efficacy and tolerability of 15 antipsychotic drugs in schizophrenia: a multiple-treatments meta-analysis. Lancet. 
2013; 382(9896): 951-962.

13. Tu B, Rich B, Labos C, Brophy JM. Coronary revascularization in diabetic patients: a systematic review and Bayesian network meta-analysis. Ann Intern Med.2014; 161(10): 724-732.

14. Wu HY, Huang JW, Lin HJ, et al. Comparative effectiveness of renin-angiotensin system blockers and other antihypertensive drugs in patients with diabetes: systematic review and bayesian network meta-analysis. BMJ. 2013; 347: f6008.

15. Moher D, Liberati A, Tetzlaff J, Altman DG. Preferred reporting items for systematic reviews and meta-analyses: the PRISMA statement. BMJ. 2009; 339: b2535. 16. Higgins JPT, Green S. Cochrane handbook for systematic reviews of interventions, version 5.1.0. Chichester: John Wiley \& Sons, 2011.

17. Harjai KJ, Raizada A, Shenoy C, et al. A comparison of contemporary definitions of contrast nephropathy in patients undergoing percutaneous coronary intervention and a proposal for a novel nephropathy grading system. Am J Cardiol 2008; 101(6): 812819.

18. Thomsen HS, Morcos SK. Contrast media and the kidney: European Society of Urogenital Radiology (ESUR) guidelines. Br J Radiol. 2003; 76(908): 513-518.

19. Ribichini F, Graziani M, Gambaro G, et al. Early creatinine shifts predict contrastinduced nephropathy and persistent renal damage after angiography. Am J Med. 2010; 123(8): 755-763.

20. Roseman M, Milette K, Bero LA, et al. Reporting of conflicts of interest in metaanalyses of trials of pharmacological treatments. JAMA. 2011;305(10):1008-1017. 
21. Warn DE, Thompson SG, Spiegelhalter DJ. Bayesian random effects meta-analysis of trials with binary outcomes: methods for the absolute risk difference and relative risk scales. Stat Med. 2002;21(11):1601-1623.

22. Cornell JE, Mulrow CD, Localio R, et al. Random-effects meta-analysis of inconsistent effects: a time for change. Ann Intern Med. 2014;160(4):267-270.

23. Brooks SP, Gelman A. General Methods for Monitoring Convergence of Iterative Simulations. J Comput Graph Stat. 1998; 7(4): 434-455.

24. Salanti G, Ades AE, Ioannidis JP. Graphical methods and numerical summaries for presenting results from multiple-treatment meta-analysis: an overview and tutorial. $J$ Clin Epidemiol. 2011; 64(2): 163-171.

25. Bucher HC, Guyatt GH, Griffith LE, Walter SD. The results of direct and indirect treatment comparisons in meta-analysis of randomized controlled trials. J Clin Epidemiol. 1997; 50(6): 683-691.

26. Tu YK, Needleman I, Chambrone L, Lu HK, Faggion CM, Jr. A Bayesian network meta-analysis on comparisons of enamel matrix derivatives, guided tissue regeneration and their combination therapies. J Clin Periodontol. 2012; 39(3): 303-314.

27. Salanti G, Marinho V, Higgins JP. A case study of multiple-treatments metaanalysis demonstrates that covariates should be considered. J Clin Epidemiol. 2009; 62(8): 857-864.

28. Dias S, Welton NJ, Sutton AJ, Caldwell DM, Lu G, Ades AE. NICE DSU technical support document 4: inconsistency in networks of evidence based on randomised controlled trials report by the decision support unit. May 2011(last updated April 2014). 
http://www.nicedsu.org.uk/TSD4\%20 Inconsistency. final. 15April2014. pdf (accessed Feb 12, 2015).

29. Dias S, Welton NJ, Caldwell DM, Ades AE. Checking consistency in mixed treatment comparison meta-analysis. Stat Med. 2010; 29(7-8): 932-944.

30. Subramaniam RM, Suarez-Cuervo C, Wilson RF, et al. Effectiveness of Prevention Strategies for Contrast-Induced Nephropathy: A Systematic Review and Meta-analysis. Ann Intern Med. 2016;164(6):406-416.

31. Xinwei J, Xianghua F, Jing Z, et al. Comparison of usefulness of simvastatin 20 $\mathrm{mg}$ versus $80 \mathrm{mg}$ in preventing contrast-induced nephropathy in patients with acute coronary syndrome undergoing percutaneous coronary intervention. Am J Cardiol. 2009; 104(4): 519-524.

32. Patti G, Ricottini E, Nusca A, et al. Short-term, high-dose Atorvastatin pretreatment to prevent contrast-induced nephropathy in patients with acute coronary syndromes undergoing percutaneous coronary intervention (from the ARMYDA-CIN [atorvastatin for reduction of myocardial damage during angioplasty--contrast-induced nephropathy] trial). Am J Cardiol. 2011; 108(1): 1-7.

33. Ozhan H, Erden I, Ordu S, et al. Efficacy of short-term high-dose atorvastatin for prevention of contrast-induced nephropathy in patients undergoing coronary angiography. Angiology. 2010; 61(7): 711-714.

34. Quintavalle C, Fiore D, De Micco F, et al. Impact of a high loading dose of atorvastatin on contrast-induced acute kidney injury. Circulation. 2012; 126(25): 30083016. 
35. Giacoppo D, Capodanno D, Capranzano P, Aruta P, Tamburino C. Meta-analysis of randomized controlled trials of preprocedural statin administration for reducing contrast-induced acute kidney injury in patients undergoing coronary catheterization. Am J Cardiol. 2014; 114(4): 541-548.

36. Zhang BC, Li WM, Xu YW. High-dose statin pretreatment for the prevention of contrast-induced nephropathy: a meta-analysis. Can J Cardiol. 2011; 27(6): 851-858.

37. Li Y, Liu Y, Fu L, Mei C, Dai B. Efficacy of short-term high-dose statin in preventing contrast-induced nephropathy: a meta-analysis of seven randomized controlled trials. PLoS One. 2012; 7(4): e34450.

38. Zhang T, Shen LH, Hu LH, He B. Statins for the prevention of contrast-induced nephropathy: a systematic review and meta-analysis. Am J Nephrol. 2011; 33(4): 344351.

39. Leoncini M, Toso A, Maioli M, Tropeano F, Villani S, Bellandi F. Early high-dose rosuvastatin for contrast-induced nephropathy prevention in acute coronary syndrome: Results from the PRATO-ACS Study (Protective Effect of Rosuvastatin and Antiplatelet Therapy On contrast-induced acute kidney injury and myocardial damage in patients with Acute Coronary Syndrome). J Am Coll Cardiol. 2014; 63(1): 71-79. 40. Han Y, Zhu G, Han L, et al. Short-term rosuvastatin therapy for prevention of contrast-induced acute kidney injury in patients with diabetes and chronic kidney disease. J Am Coll Cardiol. 2014; 63(1): 62-70.

41. Mehran R, Aymong ED, Nikolsky E, et al. A simple risk score for prediction of contrast-induced nephropathy after percutaneous coronary intervention: development 
and initial validation. J Am Coll Cardiol. 2004; 44(7): 1393-1399.

42. Haller C, Hizoh I. The cytotoxicity of iodinated radiocontrast agents on renal cells in vitro. Invest Radiol. 2004; 39(3): 149-154.

43. John S, Schneider MP, Delles C, Jacobi J, Schmieder RE. Lipid-independent effects of statins on endothelial function and bioavailability of nitric oxide in hypercholesterolemic patients. Am Heart J. 2005; 149(3): 473.

44. Kaesemeyer WH, Caldwell RB, Huang J, Caldwell RW. Pravastatin sodium activates endothelial nitric oxide synthase independent of its cholesterol-lowering actions. J Am Coll Cardiol. 1999; 33(1): 234-241.

45. Laufs U, Liao JK. Post-transcriptional regulation of endothelial nitric oxide synthase mRNA stability by Rho GTPase. J Biol Chem. 1998; 273(37): 24266-24271. 46. Ridker PM, Rifai N, Clearfield M, et al. Measurement of C-reactive protein for the targeting of statin therapy in the primary prevention of acute coronary events. $N$ Engl $J$ Med. 2001; 344(26): 1959-1965.

47. Wagner AH, Kohler T, Ruckschloss U, Just I, Hecker M. Improvement of nitric oxide-dependent vasodilatation by HMG-CoAreductase inhibitors through attenuation of endothelial superoxide anion formation. Arterioscler Thromb Vasc Biol. 2000; 20(1): $61-69$.

48. Sketch MH, Jr., Whelton A, Schollmayer E, et al. Prevention of contrast mediainduced renal dysfunction with prostaglandin E1: a randomized, double-blind, placebocontrolled study. Am J Ther. 2001; 8(3): 155-162.

49. Katholi RE, Taylor GJ, McCann WP, et al. Nephrotoxicity from contrast media: 
attenuation with theophylline. Radiology.1995; 195(1): 17-22.

50. Kapoor A, Kumar S, Gulati S, Gambhir S, Sethi RS, Sinha N. The role of theophylline in contrast-induced nephropathy: a case-control study. Nephrol Dial Transplant. 2002; 17(11): 1936-1941.

51. Dai B, Liu Y, Fu L, Li Y, Zhang J, Mei C. Effect of theophylline on prevention of contrast-induced acute kidney injury: a meta-analysis of randomized controlled trials. Am J Kidney Dis. 2012; 60(3): 360-370.

52. Upton RA. Pharmacokinetic interactions between theophylline and other medication (Part II). Clin Pharmacokinet. 1991(2); 20: 135-150.

53. Upton RA. Pharmacokinetic interactions between theophylline and other medication (Part I). Clin Pharmacokinet. 1991(1); 20: 66-80.

54. Fishbane S. N-acetylcysteine in the prevention of contrast-induced nephropathy. Clin J Am Soc Nephrol. 2008; 3(1): 281-187.

55. Brown JR, Block CA, Malenka DJ, O'Connor GT, Schoolwerth AC, Thompson CA. Sodium bicarbonate plus N-acetylcysteine prophylaxis: a meta-analysis. JACC Cardiovasc Interv. 2009; 2(11): 1116-1124.

56. Trinquart L, Attiche N, Bafeta A, Porcher R, Ravaud P. Uncertainty in Treatment Rankings: Reanalysis of Network Meta-analyses of Randomized Trials. Ann Intern Med. 2016;164(10):666-673.

57. Markota D, Markota I, Starcevic B, Tomic M, Prskalo Z, Brizic I. Prevention of contrast-induced nephropathy with Na/K citrate. Eur Heart J. 2013; 34(30): 2362-2367. 58. Erol T, Tekin A, Katircibasi MT, et al. Efficacy of allopurinol pretreatment for 
prevention of contrast-induced nephropathy: a randomized controlled trial. Int $J$ Cardiol. 2013; 167(4): 1396-1399.

59. Liu WJ, Zhang BC, Guo R, Wei YD, Li WM, Xu YW. Renoprotective effect of alprostadil in combination with statins in patients with mild to moderate renal failure undergoing coronary angiography. Chin Med J (Engl). 2013; 126(18):3475-3480. 60. Thompson SG, Higgins J. How should meta-regression analyses be undertaken and interpreted? Stat Med. 2002;21(11):1559-1573.

\section{Figure 1: Summary of trial identification and selection}

Figure 2: Network of treatment comparisons for the Bayesian network metaanalysis

The size of the nodes is proportional to the number of patients randomized to receive the treatment. Directly comparable treatments are linked with a line, the width of which is proportional to the number of trials comparing the connected treatments.

NAC, $N$-acetylcysteine.

Figure 3: Summary of the results from NMA (on the lower triangle) and traditional pairwise meta-analysis (on the upper triangle) On the lower triangle, the columndefining treatment is compared with the row-defining treatment, and odds ratios(ORs) lower than 1 favor the column-defining treatment. On the upper triangle, the rowdefining treatment is compared with the column-defining treatment, and ORs lower than 1 favor the row-defining treatment. To obtain ORs for comparisons in the opposite direction, reciprocals should be taken. Significant results are in bold. The direct comparisons within two inconsistent loops are underlined.

BIC, Bicarbonate sodium; BIC+NAC, Bicarbonate sodium plus NAC; FEN, Fenoldopam; HST, High-dose statin; HST+NAC, High-dose statin plus NAC; HYD, Hydration; LST, Low-dose statin; NAC, $N$-acetylcysteine; NAP, Natriuretic peptide; PRO, Prostaglandin; THE, Theophylline; VIT, Vitamins and its analogues.

\section{Figure 4: Forest plot for efficacy of 11 active drugs compared with hydration}

Treatments are ranked according to their OR values(vs.hydration).

CrI, credible interval. SUCRA, surface under the cumulative ranking curve measure. NAC, N-acetylcysteine. OR, odds ratio. 


\section{Supplemental Material}

\section{Table S1: Description of included studies}

ALA, alpha-lipoic acid; ANP, atrial natriuretic peptide; BNP, B-type natriuretic peptide; CI-AKI, contrast-induced acute kidney injury; Crcl, Creatinine clearance rate; eGFR, estimated glomerular filtration rate; NA, no available; NAC, $N$-acetylcysteine; $\mathrm{NaHCO}_{3}$, bicarbonate sodium; Scr, Serum creatinine.

\section{Table S2: Meta-analytic results of traditional pairwise meta-analysis}

Abbreviations: CI, confidence interval; N, number of trials; $n$, number of patients; NA, no available; NAC, $N$-acetylcysteine; OR, odds ratio, vs., versus.

${ }^{a} \tau^{2}$ represents between-study heterogeneity characterized by standard deviation.

${ }^{b}$ the meta-regression based on empirical Bayes method was used to calculate ORs and 95CIs. ORs are lower than 1 favor the former treatment of every comparison.

\section{Table S3: Evaluation of the model fit}

For a binomial likelihood each trial arm contributes 1 independent data point.

Dbar is considered as an absolute measure of fit, and is used to check formally whether a model's fit is satisfactory. This is the posterior mean of the deviance under the current model minus the deviance for the saturated mode. We can then compare the value of Dbar to the number of independent data points to check if the model fit can be improved. Leverage $\left(\boldsymbol{P}_{D}\right)$ is considered an appropriate measure of the complexity of a model that

reasonably describes the data. $P_{D}$ also is termed the effective number of parameters, and is calculated as the posterior mean of the residual deviance minus the deviance at the posterior mean of the fitted values.

Deviance Information Criterion (DIC) is the sum of the posterior mean of the residual deviance and the $P_{D}$, and provides a measure of model fit that penalises model complexity - lower values of the DIC suggest a more parsimonious model. The DIC is particularly useful for comparing different parameter models for the same likelihood and data, for example fixed and random effects models or fixed effect models with and without covariates. As shown in above table, the random consistency model is clearly more parsimonious than the other three models.

\section{Table S4: Results of sensitivity analyses}

Data are odds ratio (95\% CrI). All odds ratios use hydration as referenced agent. Heterogeneity was assessed using the posterior median between trial variance, $\tau^{2}$. Significant results are in bold.

CM, contrast media; CrI, credible interval; DM, Diabetes mellitus; SUCRA, surface under the cumulative ranking curve measure; NAC, $N$-acetylcysteine. 
Table S5: Results of meta-regression and subgroup analyses

Data are odds ratio (95\% CrI) after adjusting covariates: a. continuous variables include "Mean CM dose", "Baseline scr concentration", and "Mean age years"; b. categorical variables include "CM type (iso-, low- or high-osmolar)", "Isotonic (0.9\%) or hypotonic (0.45\%) saline hydration", "Different CI-AKI definitions (48h,72h or 120h)", "Cardiovascular diagnostic/interventional procedures or enhanced CT or not specified radiologic procedure with CM". All odds ratios use hydration as referenced agent. Heterogeneity was assessed using the posterior median between trial variance, $\tau^{2}$. Significant results are in bold.

CM, contrast media CrI, credible interval; CT, computed tomography; Scr, Serum creatinine; NAC, $N$-acetylcysteine.

\section{Figure S1: Risk of bias summary: judgements from each study}

The green symbols represent low risk of bias, the yellow symbols represent unclear risk of bias, and the red symbols represent high risk of bias. The figure was generated using

Review Manager Version 5.0.16.

\section{Figure S2: Risk of bias graph of included clinical trials}

Each methodological quality item is presented as percentages across all included studies. The figure was generated using Review Manager Version 5.0.16.

\section{Figure S3: Cumulative and non-cumulative SUCRA ranking curves}

Treatment is ranked according to SUCRA. The SUCRA would be 1 when a treatment is certain to be the best and 0 when a treatment is certain to be the worst. Higher rank indicates greater benefit probability of preventing CI-AKI.

SUCRA, surface under the cumulative ranking curve measure; NAC, $N$-acetylcysteine.

\section{Figure S4: Assessment of inconsistency}

We estimated inconsistency as the difference between direct and indirect estimates (called inconsistency factor, IF) and the corresponding 95\% confidence intervals (CI) for IF in each closed loop. The following graphs show all closed triangular loops (loops formed by three treatments) in CI-AKI outcome network. Inconsistent loops are those that present IF with 95\% CIs incompatible with zero.

There are two inconsistent loops (1-11-9 = Vitamins and its analogues - Bicarbonate sodium plus NAC - Hydration; $1-11-3=$ Vitamins and its analogues - Bicarbonate sodium plus NAC - NAC) out of 13 loops.

$1=$ Vitamins and its analogues, $2=$ Natriuretic peptide, $3=$ NAC, $4=$ Prostaglandin, 5

= High-dose statin, $6=$ Low-dose statin, $7=$ Theophylline, $8=$ Bicarbonate sodium, 9

$=$ Hydration, $10=$ Fenoldopam, $11=$ Bicarbonate sodium plus NAC, $12=$ High-dose statin plus NAC.

\section{Item S1: Study protocol}

Item S2: Statistical method

Item S3: Assessment domains of risk of bias 
Item S4: PRISMA checklist

1

2

3

4

5

7

8

9 
Table S1 Description of included studies

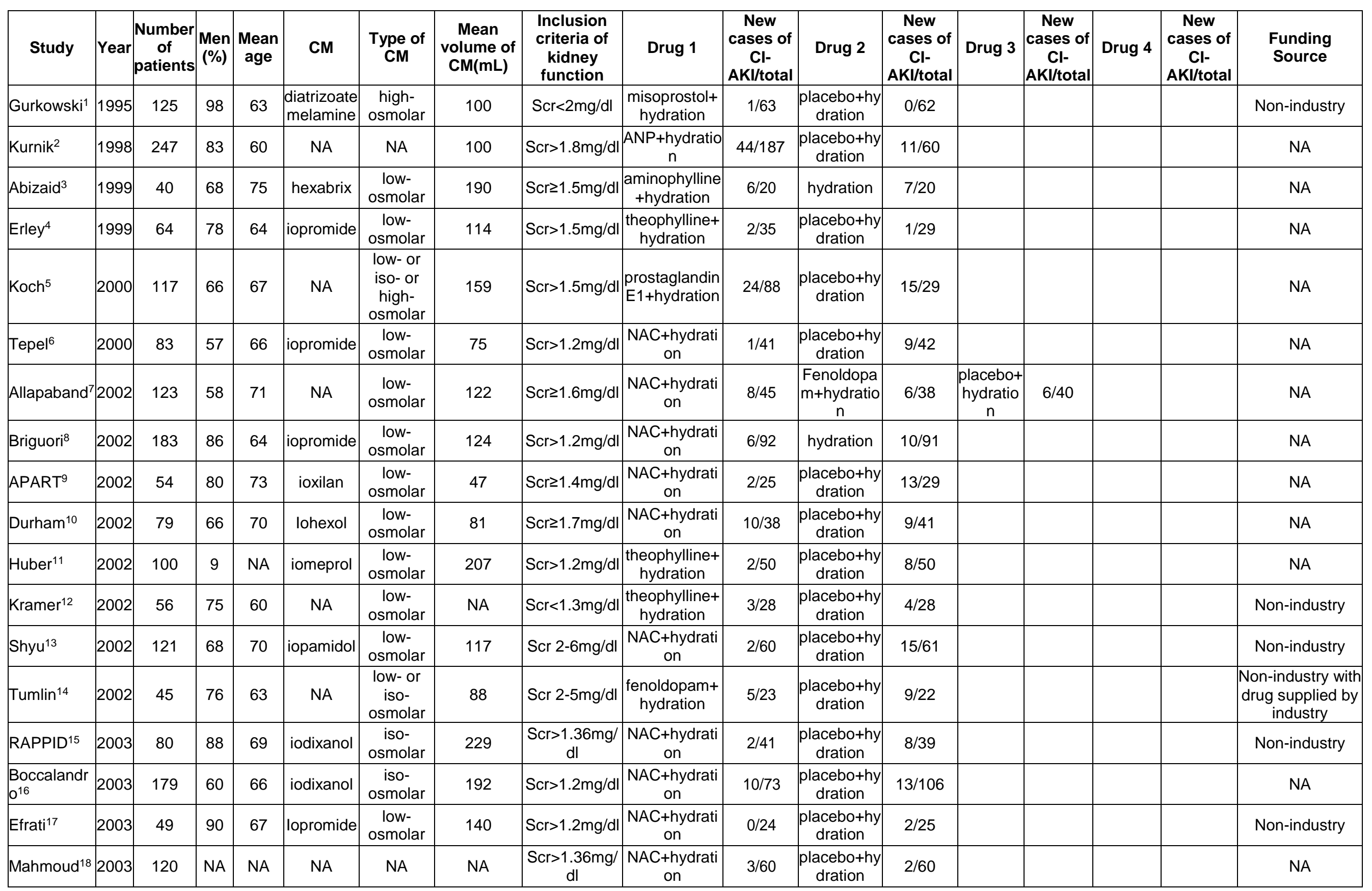




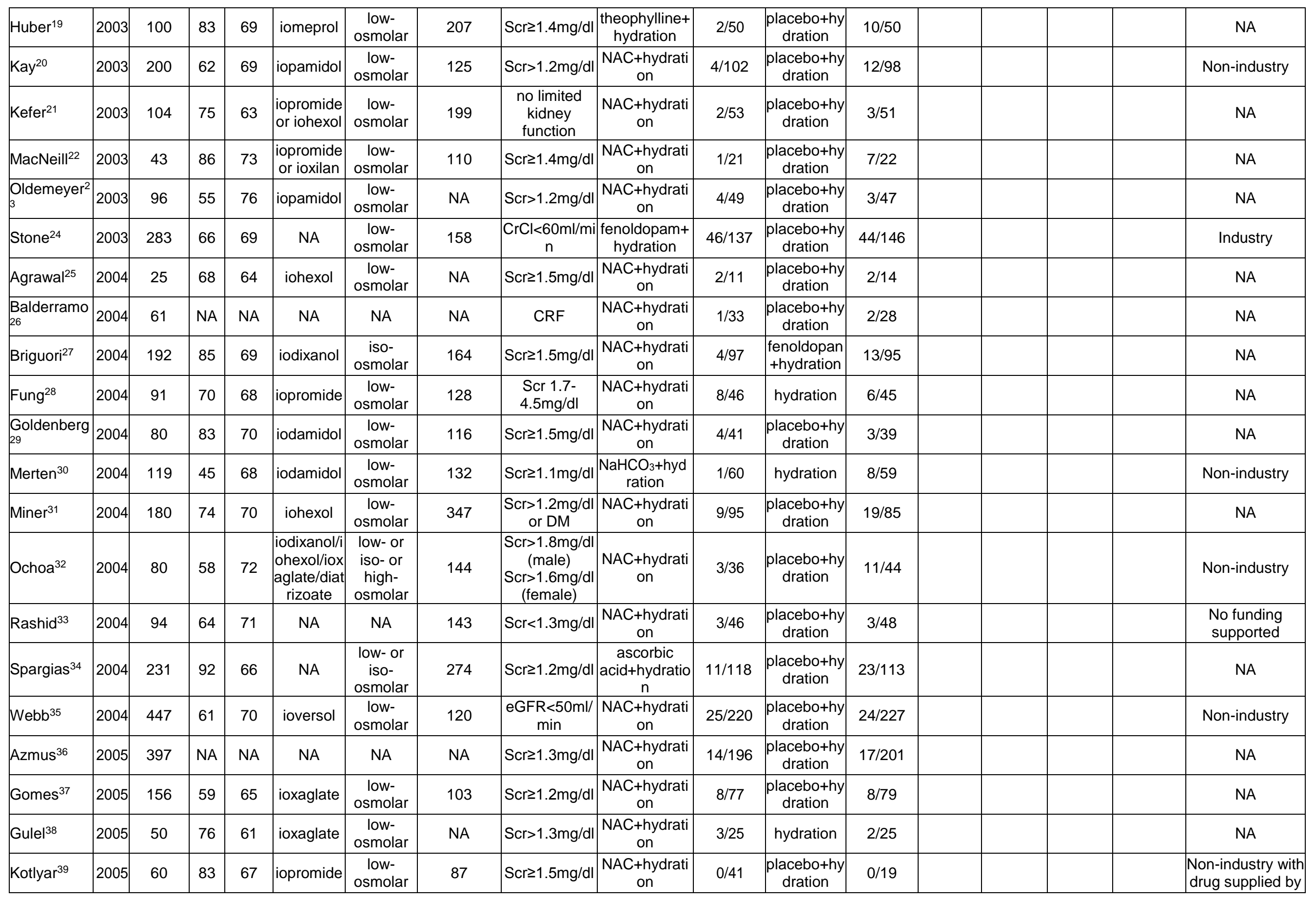




\begin{tabular}{|c|c|c|c|c|c|c|c|c|c|c|c|c|c|c|c|}
\hline & & & & & & & & & & & & & & & industry \\
\hline Coyle 40 & 2006 & 134 & 65 & 65 & NA & $\begin{array}{l}\text { low- or } \\
\text { iso- or } \\
\text { high- } \\
\text { osmolar }\end{array}$ & 93 & $\begin{array}{l}\text { no limited } \\
\text { kidney } \\
\text { function }\end{array}$ & $\begin{array}{c}\text { NAC+hydrati } \\
\text { on }\end{array}$ & $6 / 65$ & hydration & $1 / 69$ & & & NA \\
\hline Dussol ${ }^{41}$ & 2006 & 233 & 73 & 64 & NA & $\begin{array}{c}\text { low- } \\
\text { osmolar }\end{array}$ & 123 & $\begin{array}{l}\mathrm{CrCl} \mathrm{15-} \\
60 \mathrm{ml} / \mathrm{min}\end{array}$ & $\begin{array}{c}\text { theophylline+ } \\
\text { hydration }\end{array}$ & $6 / 80$ & hydration & $9 / 153$ & & & $\begin{array}{l}\text { No funding } \\
\text { supported }\end{array}$ \\
\hline Khaliliti2 & 2006 & 70 & 60 & 58 & iohexol & $\begin{array}{c}\text { low- } \\
\text { osmolar }\end{array}$ & 140 & Scr $\geq 1.2 \mathrm{mg} / \mathrm{dl}$ & $\begin{array}{c}\text { NAC+hydrati } \\
\text { on }\end{array}$ & $5 / 35$ & hydration & $12 / 35$ & & & NA \\
\hline Marenzi ${ }^{43}$ & 2006 & 354 & 95 & 63 & iohexol & $\begin{array}{c}\text { low- } \\
\text { osmolar }\end{array}$ & 266 & no dialysis & $\begin{array}{c}\text { NAC+hydrati } \\
\text { on }\end{array}$ & $27 / 235$ & $\begin{array}{c}\text { placebo+hy } \\
\text { dration }\end{array}$ & $39 / 119$ & & & Non-industry \\
\hline CAFCIN ${ }^{44}$ & 2006 & 84 & 75 & 68 & NA & $\begin{array}{l}\text { low- or } \\
\text { iso- } \\
\text { osmolar }\end{array}$ & 168 & $\mathrm{Scr} \geq 1.2 \mathrm{mg} / \mathrm{dl}$ & $\begin{array}{c}\text { NAC+hydrati } \\
\text { on }\end{array}$ & $5 / 44$ & $\begin{array}{c}\text { fenoldopan } \\
\text { +hydration }\end{array}$ & $8 / 40$ & & & NA \\
\hline Boscheri ${ }^{45}$ & 2007 & 143 & 47 & 71 & iodixanol & $\begin{array}{c}\text { iso- } \\
\text { osmolar }\end{array}$ & 106 & Scr $>1.4 \mathrm{mg} / \mathrm{dl}$ & \begin{tabular}{|c|} 
ascorbic \\
acid+hydratio \\
$n$
\end{tabular} & $5 / 74$ & $\begin{array}{c}\text { placebo+hy } \\
\text { dration }\end{array}$ & $3 / 69$ & & & NA \\
\hline $\mid \begin{array}{l}\text { REMEDIAL } \\
46\end{array}$ & 2007 & 326 & 90 & 70 & iodixanol & $\begin{array}{c}\text { iso- } \\
\text { osmolar }\end{array}$ & 174 & Scr $2-8 \mathrm{mg} / \mathrm{dl}$ & $\begin{array}{c}\text { NAC+hydrati } \\
\text { on }\end{array}$ & $12 / 111$ & $\begin{array}{c}\mathrm{NAC}+\mathrm{NaH} \\
\mathrm{CO}_{3}\end{array}$ & $2 / 108$ & $\begin{array}{c}\text { ascorbic } \\
\text { acid+NA } \\
\text { C+hydrat } \\
\text { ion }\end{array}$ & $12 / 107$ & NA \\
\hline Carbonell ${ }^{47}$ & 2007 & 216 & 76 & 62 & iopromide & $\begin{array}{c}\text { low- } \\
\text { osmolar }\end{array}$ & 188 & $\mathrm{Scr}<1.4 \mathrm{mg} / \mathrm{dl}$ & $\begin{array}{c}\text { NAC+hydrati } \\
\text { on }\end{array}$ & $11 / 107$ & $\begin{array}{c}\text { placebo+hy } \\
\text { dration }\end{array}$ & $11 / 109$ & & & NA \\
\hline Hobikoglu ${ }^{48}$ & 2007 & 81 & 72 & 62 & NA & NA & NA & $\begin{array}{l}\text { Scr } 1.4- \\
2 \mathrm{mg} / \mathrm{dl}\end{array}$ & $\begin{array}{c}\text { NAC+hydrati } \\
\text { on }\end{array}$ & $2 / 40$ & $\begin{array}{c}\text { placebo+hy } \\
\text { dration }\end{array}$ & $4 / 41$ & & & NA \\
\hline $\mathrm{Hsu}^{49}$ & 2007 & 20 & NA & NA & iohexol & $\begin{array}{c}\text { low- } \\
\text { osmolar }\end{array}$ & NA & Scr>1.6mg/dl & $\begin{array}{c}\text { NAC+hydrati } \\
\text { on }\end{array}$ & $0 / 11$ & $\begin{array}{c}\text { placebo+hy } \\
\text { dration }\end{array}$ & $5 / 9$ & & & NA \\
\hline Lawlor ${ }^{50}$ & 2007 & 78 & 69 & NA & NA & NA & 162 & Scr>1.6mg/dl & $\begin{array}{c}\text { NAC+hydrati } \\
\text { on }\end{array}$ & $4 / 53$ & $\begin{array}{c}\text { placebo+hy } \\
\text { dration }\end{array}$ & $2 / 25$ & & & Non-industry \\
\hline Masuda $^{51}$ & 2007 & 65 & 61 & 76 & iopamidol & $\begin{array}{c}\text { low- } \\
\text { osmolar }\end{array}$ & 116 & $\mathrm{Scr}>1.1 \mathrm{mg} / \mathrm{dl}$ & $\begin{array}{c}\mathrm{NaHCO}_{3}+\text { hyd } \\
\text { ration }\end{array}$ & $2 / 30$ & $\begin{array}{c}\text { placebo+hy } \\
\text { dration }\end{array}$ & $10 / 35$ & & & NA \\
\hline Ozcan 52 & 2007 & 264 & 69 & 75 & ioxaglate & $\begin{array}{c}\text { low- } \\
\text { osmolar }\end{array}$ & 110 & $\mathrm{Scr}>1.2 \mathrm{mg} / \mathrm{dl}$ & $\begin{array}{c}\text { NAC+hydrati } \\
\text { on }\end{array}$ & $11 / 88$ & $\mathrm{NaHCO}_{3}$ & $4 / 88$ & $\begin{array}{c}\text { hydratio } \\
\mathrm{n}\end{array}$ & $12 / 88$ & NA \\
\hline RENO53 & 2007 & 111 & 69 & 65 & iomeprol & $\begin{array}{c}\text { low- } \\
\text { osmolar }\end{array}$ & 285 & $\begin{array}{l}\text { no limited } \\
\text { kidney } \\
\text { function }\end{array}$ & $\begin{array}{c}\mathrm{NAC}+\mathrm{NaHCO} \\
3\end{array}$ & $1 / 56$ & $\begin{array}{c}\text { NAC+hydra } \\
\text { tion }\end{array}$ & $17 / 55$ & & & NA \\
\hline$D D^{54}$ & 2007 & 229 & 75 & 67 & iopromide & $\begin{array}{c}\text { low- } \\
\text { osmolar }\end{array}$ & 134 & \begin{tabular}{|c|} 
Scr $1.3-$ \\
$3.5 \mathrm{mg} / \mathrm{dl}$ \\
\end{tabular} & $\begin{array}{c}\text { NAC+hydrati } \\
\text { on }\end{array}$ & $6 / 114$ & hydration & $7 / 115$ & & & Non-industry \\
\hline Rajamalar ${ }^{55}$ & 2007 & 40 & 65 & 76 & iohexol & $\begin{array}{c}\text { low- } \\
\text { osmolar }\end{array}$ & 141 & $\begin{array}{c}\text { Scr }>1.4 \mathrm{mg} / \mathrm{dl} \\
\text { (male) } \\
\text { Scr }>1.3 \mathrm{mg} / \mathrm{dl} \\
\text { (female) }\end{array}$ & $\begin{array}{c}\text { NAC+hydrati } \\
\text { on }\end{array}$ & $1 / 20$ & $\begin{array}{c}\text { placebo+hy } \\
\text { dration }\end{array}$ & $2 / 20$ & & & NA \\
\hline \begin{tabular}{|l|} 
REINFORC \\
$E^{56}$
\end{tabular} & 2008 & 145 & 78 & 72 & iodixanol & $\begin{array}{c}\text { iso- } \\
\text { osmolar }\end{array}$ & 140 & $\mathrm{Scr}>1.2 \mathrm{mg} / \mathrm{dl}$ & $\mathrm{NaHCO}_{3}$ & $3 / 71$ & hydration & $2 / 74$ & & & NA \\
\hline Brar 57 & 2008 & 323 & 36 & 71 & ioxilan & $\begin{array}{c}\text { low- } \\
\text { osmolar }\end{array}$ & 132 & $\begin{array}{c}\text { eGFR }<60 \mathrm{ml} / \\
\min \end{array}$ & $\mathrm{NaHCO}_{3}$ & $30 / 165$ & hydration & $26 / 158$ & & & Non-industry \\
\hline
\end{tabular}




\begin{tabular}{|c|c|c|c|c|c|c|c|c|c|c|c|c|c|c|c|c|c|}
\hline Heng 58 & 2008 & 60 & 78 & 73 & \begin{tabular}{|c|} 
iomeprol \\
or \\
iodixanol \\
\end{tabular} & $\begin{array}{l}\text { low- or } \\
\text { iso- } \\
\text { osmolar }\end{array}$ & 203 & $\begin{array}{c}\text { eGFR }<56 \mathrm{ml} / \\
\min \end{array}$ & $\mid \begin{array}{c}\mathrm{NAC}+\mathrm{NaHCO} \\
3\end{array}$ & $1 / 28$ & $\mathrm{NaHCO}_{3}$ & $2 / 32$ & & & & & Non-industry \\
\hline Mohamed 59 & 2008 & 100 & 84 & 57 & iohexol & $\begin{array}{c}\text { low- } \\
\text { osmolar }\end{array}$ & 132 & $\begin{array}{l}\mathrm{CrCl} 40- \\
90 \mathrm{ml} / \mathrm{min}\end{array}$ & $\begin{array}{c}\text { NAC+hydrati } \\
\text { on }\end{array}$ & $2 / 49$ & $\begin{array}{c}\text { placebo+hy } \\
\text { dration }\end{array}$ & $6 / 51$ & & & & & Non-industry \\
\hline PROMISS 60 & 2008 & 236 & 72 & 66 & iodixanol & $\begin{array}{c}\text { iso- } \\
\text { osmolar }\end{array}$ & 182 & Scr $>1.1 \mathrm{mg} / \mathrm{dl}$ & $\begin{array}{c}\text { simvastatin } \\
40 m g+h y d r a t i \\
\text { on }\end{array}$ & $3 / 118$ & $\begin{array}{c}\text { placebo+hy } \\
\text { dration }\end{array}$ & $4 / 118$ & & & & & Non-industry \\
\hline Kimmel ${ }^{61}$ & 2008 & 36 & 75 & 69 & iomeprol & $\begin{array}{c}\text { low- } \\
\text { osmolar }\end{array}$ & 193 & $\mathrm{Scr} \geq 1.2 \mathrm{mg} / \mathrm{dl}$ & $\begin{array}{c}\text { NAC+hydrati } \\
\text { on }\end{array}$ & $2 / 17$ & $\begin{array}{c}\text { placebo+hy } \\
\text { dration }\end{array}$ & $1 / 19$ & & & & & $\begin{array}{l}\text { No funding } \\
\text { supported }\end{array}$ \\
\hline Masuda 63 & 2008 & 59 & 61 & 76 & iopamidol & $\begin{array}{c}\text { low- } \\
\text { osmolar }\end{array}$ & 116 & Scr $>1.1 \mathrm{mg} / \mathrm{dl}$ & $\mathrm{NaHCO}_{3}$ & $2 / 30$ & hydration & $10 / 29$ & & & & & Non-industry \\
\hline Wang 64 & 2008 & 46 & 59 & 67 & iopromide & $\begin{array}{c}\text { low- } \\
\text { osmolar }\end{array}$ & 93 & no dialysis & $\begin{array}{c}\text { NAC+hydrati } \\
\text { on }\end{array}$ & $0 / 23$ & hydration & $0 / 23$ & & & & & NA \\
\hline Amini65 & 2008 & 90 & 60 & 64 & $\begin{array}{c}\text { iohexol or } \\
\text { iodixanol }\end{array}$ & $\begin{array}{l}\text { low- or } \\
\text { iso- } \\
\text { osmolar }\end{array}$ & 120 & $\begin{array}{c}\text { Scr }>1.5 \mathrm{mg} / \mathrm{dl} \\
\text { (male) } \\
\text { Scr }>1.4 \mathrm{mg} / \mathrm{dl} \\
\text { (female) }\end{array}$ & $\begin{array}{c}\text { NAC+hydrati } \\
\text { on }\end{array}$ & $5 / 45$ & $\begin{array}{c}\text { placebo+hy } \\
\text { dration }\end{array}$ & $6 / 45$ & & & & & $\begin{array}{l}\text { No funding } \\
\text { supported }\end{array}$ \\
\hline Ferrario 67 & 2009 & 200 & 65 & 75 & iodixanol & $\begin{array}{c}\text { iso- } \\
\text { osmolar }\end{array}$ & 174 & $\begin{array}{c}\mathrm{CrCl}<55 \mathrm{ml} / \mathrm{mi} \\
\mathrm{n}\end{array}$ & $\begin{array}{c}\text { NAC+hydrati } \\
\text { on }\end{array}$ & $8 / 99$ & $\begin{array}{c}\text { placebo+hy } \\
\text { dration }\end{array}$ & $6 / 101$ & & & & & $\begin{array}{l}\text { No funding } \\
\text { supported }\end{array}$ \\
\hline $\mathrm{Kim}^{68}$ & 2009 & 108 & NA & NA & iodixanol & $\begin{array}{c}\text { iso- } \\
\text { osmolar }\end{array}$ & NA & $\underset{\mathrm{n}}{\mathrm{CrCl}<60 \mathrm{ml} / \mathrm{mi}}$ & $\begin{array}{c}\text { ALA+hydratio } \\
n\end{array}$ & $2 / 53$ & $\begin{array}{c}\text { placebo+hy } \\
\text { dration }\end{array}$ & $2 / 55$ & & & & & NA \\
\hline NASPI69 & 2009 & 174 & 78 & 65 & iodixanol & $\begin{array}{c}\text { iso- } \\
\text { osmolar }\end{array}$ & 210 & Scr $\geq 1.1 \mathrm{mg} / \mathrm{dl}$ & $\begin{array}{c}\text { NAC+hydrati } \\
\text { on }\end{array}$ & $1 / 83$ & $\begin{array}{c}\text { ascorbic } \\
\text { acid+hydrat } \\
\text { ion }\end{array}$ & $4 / 91$ & & & & & Non-industry \\
\hline Marikawa ${ }^{70}$ & 2009 & 254 & 72 & 74 & iomeprol & $\begin{array}{c}\text { low- } \\
\text { osmolar }\end{array}$ & 140 & $\mathrm{Scr} \geq 1.3 \mathrm{mg} / \mathrm{dl}$ & $\begin{array}{c}\text { ANP+hydratio } \\
n\end{array}$ & $4 / 126$ & hydration & $15 / 128$ & & & & & NA \\
\hline Pakfetrat $^{71}$ & 2009 & 192 & 61 & 58 & iodixanol & $\begin{array}{c}\text { iso- } \\
\text { osmolar }\end{array}$ & 63 & no dialysis & $\mathrm{NaHCO}_{3}$ & $4 / 96$ & hydration & $12 / 96$ & & & & & Non-industry \\
\hline Ratcliffe $^{72}$ & 2009 & 78 & 60 & 66 & iodixanol & $\begin{array}{c}\text { iso- } \\
\text { osmolar }\end{array}$ & 150 & $\begin{array}{c}\text { Scr }>1.5 \mathrm{mg} / \mathrm{dl} \\
\text { (male) } \\
\text { Scr }>1.3 \mathrm{mg} / \mathrm{dl} \\
\text { (female) }\end{array}$ & hydration & $1 / 15$ & $\begin{array}{c}\text { NAC+hydra } \\
\text { tion }\end{array}$ & $1 / 21$ & $\mathrm{NaHCO}_{3}$ & $2 / 19$ & $\mid \begin{array}{c}\mathrm{NAC}+\mathrm{Na} \\
\mathrm{HCO}_{3}\end{array}$ & $1 / 23$ & NA \\
\hline Spargias $^{73}$ & 2009 & 208 & 89 & 71 & NA & $\begin{array}{l}\text { low- or } \\
\text { iso- } \\
\text { osmolar }\end{array}$ & 203 & $\mathrm{Scr} \geq 1.4 \mathrm{mg} / \mathrm{dl}$ & $\begin{array}{l}\text { iloprost+hydr } \\
\text { ation }\end{array}$ & $8 / 103$ & $\begin{array}{c}\text { placebo+hy } \\
\text { dration }\end{array}$ & $23 / 105$ & & & & & Industry \\
\hline$\prod_{75}^{T a s a n a t o n g}$ & 2009 & 103 & 74 & 67 & iopromide & $\begin{array}{c}\text { low- } \\
\text { osmolar }\end{array}$ & 141 & $\mathrm{Scr} \geq 1.2 \mathrm{mg} / \mathrm{dl}$ & \begin{tabular}{|c|} 
alpha \\
tocopherol+h \\
ydration
\end{tabular} & $12 / 52$ & $\begin{array}{c}\text { placebo+hy } \\
\text { dration }\end{array}$ & $3 / 51$ & & & & & NA \\
\hline Farahani ${ }^{76}$ & 2009 & 265 & 83 & 64 & iohexol & $\begin{array}{c}\text { low- } \\
\text { osmolar }\end{array}$ & 114 & $\mathrm{Scr} \geq 1.5 \mathrm{mg} / \mathrm{dl}$ & $\begin{array}{c}\mathrm{NaHCO}_{3}+\text { hyd } \\
\text { ration }\end{array}$ & $8 / 130$ & hydration & $11 / 135$ & & & & & Non-industry \\
\hline
\end{tabular}




\begin{tabular}{|c|c|c|c|c|c|c|c|c|c|c|c|c|c|c|c|}
\hline $\mathrm{Jia}^{77}$ & 2009 & 228 & 36 & 66 & $\begin{array}{c}\text { iohexol or } \\
\text { iodixanol }\end{array}$ & $\begin{array}{l}\text { low- or } \\
\text { iso- } \\
\text { osmolar }\end{array}$ & NA & no dialysis & \begin{tabular}{|c|} 
simvastatin \\
$20 m g+h y d r a t i$ \\
on
\end{tabular} & $18 / 113$ & $\begin{array}{c}\text { simvastatin } \\
80 \mathrm{mg}+\mathrm{hydr} \\
\text { ation }\end{array}$ & $6 / 115$ & & & NA \\
\hline Zhou ${ }^{78}$ & 2009 & 100 & 59 & 60 & iopamidol & $\begin{array}{l}\text { low- } \\
\text { osmolar }\end{array}$ & 116 & Scr $<1.7 \mathrm{mg} / \mathrm{dl}$ & $\begin{array}{c}\text { atorvastatin } \\
80 \mathrm{mg}+\text { hydrati } \\
\text { on }\end{array}$ & $0 / 50$ & $\begin{array}{c}\text { atorvastatin } \\
10 \mathrm{mg}+\mathrm{hydr} \\
\text { ation }\end{array}$ & $3 / 50$ & & & NA \\
\hline Hakan 79 & 2010 & 60 & 70 & 61 & iobitridol & $\begin{array}{c}\text { low- } \\
\text { osmolar }\end{array}$ & NA & $\begin{array}{l}\text { no limited } \\
\text { kidney } \\
\text { function }\end{array}$ & $\begin{array}{c}\text { NAC+hydrati } \\
\text { on }\end{array}$ & $1 / 30$ & hydration & $1 / 30$ & & & $\begin{array}{l}\text { No funding } \\
\text { supported }\end{array}$ \\
\hline Carbonell ${ }^{80}$ & 2010 & 81 & 80 & 70 & iopromide & $\begin{array}{c}\text { low- } \\
\text { osmolar }\end{array}$ & 160 & $\mathrm{Scr} \geq 1.4 \mathrm{mg} / \mathrm{dl}$ & $\begin{array}{c}\text { NAC+hydrati } \\
\text { on }\end{array}$ & $2 / 39$ & $\begin{array}{c}\text { placebo+hy } \\
\text { dration }\end{array}$ & $10 / 42$ & & & NA \\
\hline Castini 81 & 2010 & 156 & 88 & 72 & iodixanol & $\begin{array}{c}\text { iso- } \\
\text { osmolar }\end{array}$ & 195 & $\mathrm{Scr} \geq 1.2 \mathrm{mg} / \mathrm{dl}$ & $\begin{array}{c}\text { NAC+hydrati } \\
\text { on }\end{array}$ & $9 / 53$ & $\mathrm{NaHCO}_{3}$ & $7 / 52$ & \begin{tabular}{|c} 
hydratio \\
$\mathrm{n}$
\end{tabular} & $7 / 51$ & NA \\
\hline ENABLE $^{82}$ & 2010 & 166 & 73 & 62 & \begin{tabular}{|c|} 
iodixanol \\
or \\
iopamidol \\
or \\
iobitridol \\
\end{tabular} & $\begin{array}{l}\text { low- or } \\
\text { iso- } \\
\text { osmolar }\end{array}$ & 209 & $\begin{array}{c}\mathrm{Scr}<1.4 \mathrm{mg} / \mathrm{dl} \\
\quad(\mathrm{male}) \\
\mathrm{Scr}<1.2 \mathrm{mg} / \mathrm{dl} \\
\text { (female) }\end{array}$ & $\begin{array}{c}\text { NAC+hydrati } \\
\text { on }\end{array}$ & $3 / 80$ & hydration & $7 / 86$ & & & NA \\
\hline Kinbara ${ }^{83}$ & 2010 & 45 & 62 & 70 & iopamidol & $\begin{array}{c}\text { low- } \\
\text { osmolar }\end{array}$ & 143 & no dialysis & \begin{tabular}{|c|} 
NAC+hydrati \\
on \\
\end{tabular} & $0 / 15$ & $\begin{array}{c}\text { aminophylli } \\
\text { ne }\end{array}$ & $0 / 15$ & \begin{tabular}{|c|} 
hydratio \\
$\mathrm{n}$
\end{tabular} & $0 / 15$ & $\begin{array}{l}\text { No funding } \\
\text { supported }\end{array}$ \\
\hline Matejka ${ }^{85}$ & 2010 & 56 & 61 & 75 & iodixanol & $\begin{array}{c}\text { iso- } \\
\text { osmolar }\end{array}$ & 95 & $\mathrm{Scr} \geq 1.5 \mathrm{mg} / \mathrm{dl}$ & $\begin{array}{c}\text { theophylline+ } \\
\text { hydration }\end{array}$ & $3 / 31$ & $\begin{array}{c}\text { placebo+hy } \\
\text { dration }\end{array}$ & $0 / 25$ & & & Non-industry \\
\hline Hakan ${ }^{86}$ & 2010 & 130 & 41 & 55 & iopamidol & $\begin{array}{l}\text { low- } \\
\text { osmolar }\end{array}$ & 95 & $\mathrm{Scr} \leq 1.5 \mathrm{mg} / \mathrm{dl}$ & $\begin{array}{c}\text { atorvastatin } \\
\text { 80mg+hydrati } \\
\text { on+NAC }\end{array}$ & $2 / 60$ & $\begin{array}{c}\text { NAC+hydra } \\
\text { tion }\end{array}$ & $7 / 70$ & & & $\begin{array}{l}\text { No funding } \\
\text { supported }\end{array}$ \\
\hline Rohani87 & 2010 & 60 & NA & 62 & iohexol & $\begin{array}{c}\text { low- } \\
\text { osmolar }\end{array}$ & 205 & $\mathrm{Scr}>1.3 \mathrm{mg} / \mathrm{dl}$ & $\begin{array}{c}\text { aminophylline } \\
\text { +hydration }\end{array}$ & $4 / 30$ & $\begin{array}{c}\text { placebo+hy } \\
\text { dration }\end{array}$ & $6 / 30$ & & & NA \\
\hline Sar 88 & 2010 & 45 & 53 & 57 & iohexol & $\begin{array}{c}\text { low- } \\
\text { osmolar }\end{array}$ & NA & $\mathrm{Scr}<1.2 \mathrm{mg} / \mathrm{dl}$ & $\begin{array}{c}\text { NAC+hydrati } \\
\text { on }\end{array}$ & $0 / 25$ & hydration & $2 / 20$ & & & $\begin{array}{l}\text { No funding } \\
\text { supported }\end{array}$ \\
\hline LIPSIA 89 & 2010 & 249 & 68 & 68 & iopromide & $\begin{array}{c}\text { low- } \\
\text { osmolar }\end{array}$ & 170 & no dialysis & $\begin{array}{c}\text { NAC+hydrati } \\
\text { on }\end{array}$ & $18 / 126$ & $\begin{array}{c}\text { placebo+hy } \\
\text { dration }\end{array}$ & $25 / 123$ & & & NA \\
\hline Toso 90 & 2010 & 304 & 64 & 76 & iodixanol & $\begin{array}{c}\text { iso- } \\
\text { osmolar }\end{array}$ & 158 & $\begin{array}{c}\mathrm{CrCl}<60 \mathrm{ml} / \mathrm{mi} \\
\mathrm{n}\end{array}$ & $\begin{array}{c}\text { atorvastatin } \\
\text { 80mg+hydrati } \\
\text { on+NAC }\end{array}$ & $15 / 152$ & $\begin{array}{c}\text { NAC+hydra } \\
\text { tion }\end{array}$ & $16 / 152$ & & & NA \\
\hline Farahani91 & 2010 & 72 & 79 & 62 & iohexol & $\begin{array}{c}\text { low- } \\
\text { osmolar }\end{array}$ & 118 & $\mathrm{Scr}>1.5 \mathrm{mg} / \mathrm{dl}$ & $\begin{array}{c}\mathrm{NaHCO}_{3}+\text { hyd } \\
\text { ration }\end{array}$ & $2 / 36$ & hydration & $2 / 36$ & & & Non-industry \\
\hline Zhang ${ }^{92}$ & 2010 & 149 & 70 & 64 & $\begin{array}{c}\text { iohexol or } \\
\text { iodixanol }\end{array}$ & $\begin{array}{l}\text { low- or } \\
\text { iso- } \\
\text { osmolar }\end{array}$ & 186 & $\begin{array}{l}\text { no limited } \\
\text { kidney } \\
\text { function }\end{array}$ & $\begin{array}{c}\text { BNP+hydratio } \\
n\end{array}$ & $12 / 74$ & $\begin{array}{c}\text { placebo+hy } \\
\text { dration }\end{array}$ & $24 / 75$ & & & Non-industry \\
\hline $\mathrm{Kim}^{94}$ & 2011 & 191 & NA & NA & iodixanol & $\begin{array}{c}\text { iso- } \\
\text { osmolar }\end{array}$ & NA & NA & $\begin{array}{c}\text { atorvastatin8 } \\
\text { Omg+hydratio }\end{array}$ & $5 / 92$ & hydration & $4 / 99$ & & & NA \\
\hline
\end{tabular}




\begin{tabular}{|c|c|c|c|c|c|c|c|c|c|c|c|c|c|c|c|c|c|}
\hline & & & & & & & & & $\mathrm{n}$ & & & & & & & & \\
\hline $\begin{array}{l}\text { PREVENT }_{5}^{9} \\
5\end{array}$ & 2011 & 375 & 71 & 68 & iodixanol & $\begin{array}{c}\text { iso- } \\
\text { osmolar }\end{array}$ & 117 & $\mathrm{Scr} \geq 1.1 \mathrm{mg} / \mathrm{dl}$ & $\begin{array}{c}\text { NAC+hydrati } \\
\text { on }\end{array}$ & $10 / 187$ & $\begin{array}{c}\mathrm{NaHCO}_{3}+\mathrm{N} \\
\mathrm{AC}\end{array}$ & $17 / 188$ & & & & & Non-industry \\
\hline Maioli96 & 2011 & 300 & 74 & 65 & iodixanol & $\begin{array}{c}\text { iso- } \\
\text { osmolar }\end{array}$ & 216 & no dialysis & $\mathrm{NaHCO}_{3}$ & $18 / 150$ & hydration & $34 / 150$ & & & & & $\begin{array}{l}\text { No funding } \\
\text { supported }\end{array}$ \\
\hline Motohiro 97 & 2011 & 155 & 70 & 73 & iopamidol & $\begin{array}{c}\text { low- } \\
\text { osmolar }\end{array}$ & 135 & $\begin{array}{c}\text { eGFR }<60 \mathrm{ml} / \\
\min \end{array}$ & $\begin{array}{c}\mathrm{NaHCO}_{3}+\text { hyd } \\
\text { ration }\end{array}$ & $2 / 78$ & hydration & $10 / 77$ & & & & & NA \\
\hline ARMYDA $^{98}$ & 2011 & 241 & 78 & 66 & iobitridol & $\begin{array}{c}\text { low- } \\
\text { osmolar }\end{array}$ & 211 & Scr $<3 \mathrm{mg} / \mathrm{dl}$ & $\begin{array}{c}\text { atorvastatin8 } \\
\text { Omg+hydratio } \\
\mathrm{n}\end{array}$ & $6 / 120$ & $\begin{array}{c}\text { placebo+hy } \\
\text { dration }\end{array}$ & $16 / 121$ & & & & & NA \\
\hline Sadat ${ }^{99}$ & 2011 & 40 & NA & 72 & iopamidol & $\begin{array}{c}\text { low- } \\
\text { osmolar }\end{array}$ & 73 & no dialysis & $\begin{array}{c}\text { NAC+hydrati } \\
\text { on }\end{array}$ & $1 / 21$ & hydration & $3 / 19$ & & & & & $\begin{array}{l}\text { No funding } \\
\text { supported }\end{array}$ \\
\hline Tanaka ${ }^{100}$ & 2011 & 76 & 82 & 62 & iopamidol & $\begin{array}{c}\text { low- } \\
\text { osmolar }\end{array}$ & 211 & no dialysis & $\begin{array}{c}\text { NAC+hydrati } \\
\text { on }\end{array}$ & $2 / 38$ & hydration & $5 / 38$ & & & & & NA \\
\hline Ueda 101 & 2011 & 59 & 78 & 76 & $\begin{array}{l}\text { iopamidol } \\
\text { or iohexol }\end{array}$ & $\begin{array}{c}\text { low- } \\
\text { osmolar }\end{array}$ & 110 & $\mathrm{Scr} \geq 1.1 \mathrm{mg} / \mathrm{dl}$ & $\mathrm{NaHCO}_{3}$ & $1 / 30$ & hydration & $8 / 29$ & & & & & NA \\
\hline Aslanger ${ }^{102}$ & 2012 & 312 & 77 & 56 & ioxaglate & $\begin{array}{c}\text { low- } \\
\text { osmolar }\end{array}$ & 200 & no dialysis & $\begin{array}{c}\text { NAC+hydrati } \\
\text { on }\end{array}$ & $51 / 213$ & $\begin{array}{c}\text { placebo+hy } \\
\text { dration }\end{array}$ & 23/99 & & & & & $\begin{array}{l}\text { No funding } \\
\text { supported }\end{array}$ \\
\hline Bilasy ${ }^{103}$ & 2012 & 60 & 60 & 57 & iopamidol & $\begin{array}{c}\text { low- } \\
\text { osmolar }\end{array}$ & 117 & no dialysis & $\begin{array}{c}\text { theophylline+ } \\
\text { hydration+NA } \\
\text { C }\end{array}$ & $0 / 30$ & $\begin{array}{c}\text { NAC+hydra } \\
\text { tion }\end{array}$ & $6 / 30$ & & & & & $\begin{array}{l}\text { No funding } \\
\text { supported }\end{array}$ \\
\hline Cao ${ }^{104}$ & 2012 & 180 & 57 & 63 & NA & NA & 161 & no dialysis & $\begin{array}{c}\text { atorvastatin4 } \\
\text { Omg+hydratio } \\
\mathrm{n}\end{array}$ & $6 / 90$ & $\begin{array}{c}\text { atorvastatin } \\
20 \mathrm{mg}+\mathrm{hydr} \\
\text { ation }\end{array}$ & $18 / 90$ & & & & & Non-industry \\
\hline Gomes ${ }^{105}$ & 2012 & 301 & 72 & 64 & ioxaglate & $\begin{array}{c}\text { low- } \\
\text { osmolar }\end{array}$ & 125 & $\mathrm{Scr} \geq 1.1 \mathrm{mg} / \mathrm{dl}$ & $\mathrm{NaHCO}_{3}$ & $9 / 150$ & hydration & 9/151 & & & & & $\begin{array}{l}\text { No funding } \\
\text { supported }\end{array}$ \\
\hline $\begin{array}{l}\text { Gunebakm } \\
\mathrm{az}^{106}\end{array}$ & 2012 & 80 & 68 & 66 & iopromide & $\begin{array}{c}\text { low- } \\
\text { osmolar }\end{array}$ & 63 & $\mathrm{Scr} \geq 1.2 \mathrm{mg} / \mathrm{dl}$ & $\begin{array}{c}\text { NAC+hydrati } \\
\text { on }\end{array}$ & $9 / 40$ & hydration & $11 / 40$ & & & & & $\begin{array}{l}\text { No funding } \\
\text { supported }\end{array}$ \\
\hline Hafiz ${ }^{107}$ & 2012 & 320 & 57 & 74 & \begin{tabular}{|c|} 
iodixanol \\
or \\
iopamidol \\
or ioversol
\end{tabular} & $\begin{array}{c}\text { low- or } \\
\text { iso- } \\
\text { osmolar }\end{array}$ & 105 & $\begin{array}{c}\text { eGFR }<50 \mathrm{ml} / \\
\min \end{array}$ & hydration & $11 / 80$ & $\begin{array}{c}\text { NAC+hydra } \\
\text { tion }\end{array}$ & $8 / 81$ & $\mathrm{NaHCO}_{3}$ & $6 / 79$ & $\left|\begin{array}{c}\mathrm{NaHCO}_{3} \\
+\mathrm{NAC}\end{array}\right|$ & $8 / 80$ & $\begin{array}{l}\text { No funding } \\
\text { supported }\end{array}$ \\
\hline Jaffery ${ }^{108}$ & 2012 & 398 & 61 & 65 & iodixanol & $\begin{array}{c}\text { iso- } \\
\text { osmolar }\end{array}$ & 167 & no dialysis & $\begin{array}{c}\text { NAC+hydrati } \\
\text { on }\end{array}$ & $33 / 206$ & $\begin{array}{c}\text { placebo+hy } \\
\text { dration }\end{array}$ & $25 / 192$ & & & & & $\begin{array}{l}\text { No funding } \\
\text { supported }\end{array}$ \\
\hline Kitzler ${ }^{109}$ & 2012 & 30 & 57 & 75 & iopromide & $\begin{array}{l}\text { low- } \\
\text { osmolar }\end{array}$ & NA & \begin{tabular}{|c|} 
Scr $>1.3 \mathrm{mg} / \mathrm{dl}$ \\
(male) \\
Scr $>1.1 \mathrm{mg} / \mathrm{dl}$ \\
(female)
\end{tabular} & $\begin{array}{c}\text { NAC+hydrati } \\
\text { on }\end{array}$ & $0 / 10$ & $\begin{array}{c}\text { vitamin } \\
\text { E+hydratio } \\
n\end{array}$ & $0 / 10$ & $\begin{array}{c}\text { placebo+ } \\
\text { hydratio } \\
n\end{array}$ & $0 / 10$ & & & Non-industry \\
\hline Klima ${ }^{110}$ & 2012 & 258 & 64 & 77 & NA & NA & 100 & $\mid \begin{array}{c}\text { Scr }>1.3 \mathrm{mg} / \mathrm{dl} \\
\text { (male) } \\
\text { Scr }>1.1 \mathrm{mg} / \mathrm{dl} \\
\text { (female) }\end{array}$ & $\mathrm{NaHCO}_{3}$ & $16 / 169$ & hydration & $1 / 89$ & & & & & Non-industry \\
\hline CASIS ${ }^{111}$ & 2012 & 220 & 78 & 64 & iohexol & $\begin{array}{c}\text { low- } \\
\text { osmolar }\end{array}$ & 127 & $S c r \geq 1.1 \mathrm{mg} / \mathrm{dl}$ & \begin{tabular}{|c|}
$\begin{array}{c}\text { NAC+hydrati } \\
\text { on }\end{array}$ \\
\end{tabular} & $2 / 80$ & hydration & $19 / 140$ & & & & & NA \\
\hline Li112 & 2012 & 161 & 76 & 66 & iohexol & $\begin{array}{c}\text { low- } \\
\text { osmolar }\end{array}$ & 102 & $\begin{array}{c}\text { normal kidney } \\
\text { function }\end{array}$ & \begin{tabular}{|l|} 
atorvastatin8 \\
Omg+hydratio
\end{tabular} & $2 / 78$ & $\begin{array}{c}\text { placebo+hy } \\
\text { dration }\end{array}$ & $13 / 83$ & & & & & NA \\
\hline
\end{tabular}




\begin{tabular}{|c|c|c|c|c|c|c|c|c|c|c|c|c|c|c|c|}
\hline & & & & & & & & & $\mathrm{n}$ & & & & & & \\
\hline Okumura ${ }^{113}$ & 2012 & 112 & 68 & 74 & iohexol & $\begin{array}{c}\text { low- } \\
\text { osmolar }\end{array}$ & 66 & $\begin{array}{c}\text { eGFR }<60 \mathrm{ml} / \\
\min \end{array}$ & $\begin{array}{c}\text { carperitide+h } \\
\text { ydration }\end{array}$ & $5 / 59$ & hydration & $3 / 53$ & & & $\begin{array}{l}\text { No funding } \\
\text { supported }\end{array}$ \\
\hline NAPLE II ${ }^{114}$ & 2012 & 410 & 54 & 70 & iodixanol & $\begin{array}{c}\text { iso- } \\
\text { osmolar }\end{array}$ & 181 & $\begin{array}{c}\text { no limited } \\
\text { kidney } \\
\text { function }\end{array}$ & $\begin{array}{c}\text { atorvastatin8 } \\
\text { Omg+hydratio } \\
\mathrm{n}+\mathrm{NAC}\end{array}$ & $7 / 202$ & $\begin{array}{c}\mathrm{NaHCO}_{3}+\mathrm{N} \\
\mathrm{AC}\end{array}$ & $12 / 208$ & & & Non-industry \\
\hline Zhou 115 & 2012 & 156 & 84 & 71 & NA & NA & 135 & Scr $\geq 1.1 \mathrm{mg} / \mathrm{dl}$ & $\begin{array}{c}\text { ascorbic } \\
\text { acid+hydratio } \\
\mathrm{n}\end{array}$ & $6 / 82$ & $\begin{array}{c}\text { placebo+hy } \\
\text { dration }\end{array}$ & $4 / 74$ & & & $\begin{array}{l}\text { No funding } \\
\text { supported }\end{array}$ \\
\hline Albabtain ${ }^{116}$ & 2013 & 185 & 73 & 61 & ioxaglate & $\begin{array}{l}\text { low- } \\
\text { osmolar }\end{array}$ & 88 & Scr $\geq 1.3 \mathrm{mg} / \mathrm{dl}$ & $\begin{array}{c}\text { ascorbic } \\
\text { acid+hydratio } \\
n\end{array}$ & $2 / 57$ & $\begin{array}{c}\text { NAC+hydra } \\
\text { tion }\end{array}$ & $5 / 62$ & $\begin{array}{c}\text { placebo+ } \\
\text { hydratio } \\
n\end{array}$ & $5 / 66$ & NA \\
\hline Boucek $^{117}$ & 2013 & 120 & 75 & 65 & NA & $\begin{array}{c}\text { low- } \\
\text { osmolar }\end{array}$ & 110 & Scr $\geq 1.1 \mathrm{mg} / \mathrm{dl}$ & $\mathrm{NaHCO}_{3}$ & $7 / 61$ & hydration & $5 / 59$ & & & $\begin{array}{l}\text { No funding } \\
\text { supported }\end{array}$ \\
\hline Brueck $^{118}$ & 2013 & 483 & 64 & 75 & iopromide & $\begin{array}{c}\text { low- } \\
\text { osmolar }\end{array}$ & 112 & Scr $\geq 1.3 \mathrm{mg} / \mathrm{dl}$ & $\begin{array}{c}\text { ascorbic } \\
\text { acid+hydratio } \\
n\end{array}$ & $24 / 98$ & $\begin{array}{c}\text { NAC+hydra } \\
\text { tion }\end{array}$ & $53 / 192$ & $\mid \begin{array}{c}\text { placebo+ } \\
\text { hydratio } \\
n\end{array}$ & $62 / 193$ & $\begin{array}{l}\text { No funding } \\
\text { supported }\end{array}$ \\
\hline Cicek $^{119}$ & 2013 & 78 & 60 & 65 & iohexol & $\begin{array}{c}\text { low- } \\
\text { osmolar }\end{array}$ & 78 & $\mathrm{Scr}<1.5 \mathrm{mg} / \mathrm{dl}$ & $\begin{array}{c}\text { ALA+hydratio } \\
n\end{array}$ & $3 / 39$ & hydration & $3 / 39$ & & & $\begin{array}{l}\text { No funding } \\
\text { supported }\end{array}$ \\
\hline Drorsak $^{120}$ & 2013 & 81 & 73 & 71 & iopamidol & $\begin{array}{c}\text { low- } \\
\text { osmolar }\end{array}$ & 138 & $\mathrm{Scr} \geq 1.2 \mathrm{mg} / \mathrm{dl}$ & $\begin{array}{c}\text { ascorbic } \\
\text { acid+hydratio } \\
\mathrm{n}\end{array}$ & $2 / 40$ & $\begin{array}{c}\text { placebo+hy } \\
\text { dration }\end{array}$ & $3 / 41$ & & & $\begin{array}{l}\text { No funding } \\
\text { supported }\end{array}$ \\
\hline Ricardo 121 & 2013 & 123 & 72 & 66 & ioversol & $\begin{array}{c}\text { low- } \\
\text { osmolar }\end{array}$ & 192 & $\mathrm{Scr} \geq 1.2 \mathrm{mg} / \mathrm{dl}$ & $\mathrm{NaHCO}_{3}$ & $15 / 42$ & $\begin{array}{c}\mathrm{NAC}+\mathrm{NaH} \\
\mathrm{CO}_{3}\end{array}$ & $3 / 43$ & $\begin{array}{l}\text { NAC+hy } \\
\text { dration }\end{array}$ & $6 / 38$ & $\begin{array}{l}\text { No funding } \\
\text { supported }\end{array}$ \\
\hline Huil22 & 2013 & 182 & NA & NA & NA & NA & NA & NA & $\begin{array}{c}\text { NAC+hydrati } \\
\text { on }\end{array}$ & $4 / 92$ & $\begin{array}{c}\text { placebo+hy } \\
\text { dration }\end{array}$ & $10 / 90$ & & & NA \\
\hline ALIVE $^{123}$ & 2013 & 202 & 50 & 72 & \begin{tabular}{|c|} 
iodixanol \\
or \\
iopromide \\
or \\
iobitridol \\
\end{tabular} & $\begin{array}{l}\text { low- or } \\
\text { iso- } \\
\text { osmolar }\end{array}$ & 170 & $\begin{array}{c}\mathrm{CrCl} \leq 60 \mathrm{ml} / \mathrm{mi} \\
\mathrm{n}\end{array}$ & $\mid \begin{array}{c}\text { ALA+hydratio } \\
n\end{array}$ & $3 / 100$ & hydration & $7 / 102$ & & & Non-industry \\
\hline Koc $^{124}$ & 2013 & 195 & 52 & 62 & NA & NA & 90 & Scr $<3 \mathrm{mg} / \mathrm{dl}$ & $\mathrm{NaHCO}_{3}$ & $15 / 94$ & hydration & $6 / 101$ & & & NA \\
\hline Maaz ${ }^{125}$ & 2013 & 160 & NA & NA & NA & NA & NA & NA & $\begin{array}{c}\text { atorvastatin8 } \\
\text { Omg+NAC+h } \\
\text { ydration }\end{array}$ & $2 / 80$ & $\begin{array}{c}\text { NAC+hydra } \\
\text { tion }\end{array}$ & $9 / 80$ & & & NA \\
\hline Miao $^{126}$ & 2013 & 330 & 77 & 79 & iohexol & $\begin{array}{c}\text { low- } \\
\text { osmolar }\end{array}$ & NA & $\mathrm{Scr}<3 \mathrm{mg} / \mathrm{dl}$ & $\begin{array}{c}\text { alprostadil+hy } \\
\text { dration }\end{array}$ & $14 / 154$ & $\begin{array}{c}\text { placebo+hy } \\
\text { dration }\end{array}$ & $39 / 176$ & & & Non-industry \\
\hline Poletti ${ }^{127}$ & 2013 & 110 & 50 & 78 & iohexol & $\begin{array}{c}\text { low- } \\
\text { osmolar }\end{array}$ & 118 & $\underset{\mathrm{n}}{\mathrm{CrCl} \leq 60 \mathrm{ml} / \mathrm{mi}}$ & $\begin{array}{c}\text { NAC+hydrati } \\
\text { on }\end{array}$ & $8 / 52$ & $\begin{array}{c}\text { placebo+hy } \\
\text { dration }\end{array}$ & $10 / 58$ & & & NA \\
\hline $\begin{array}{l}\text { Tasanarong } \\
128\end{array}$ & 2013 & 305 & 72 & 67 & iopromide & $\begin{array}{c}\text { low- } \\
\text { osmolar }\end{array}$ & 135 & $\begin{array}{c}\text { eGFR } \leq 60 \mathrm{ml} / \\
\min \end{array}$ & $\begin{array}{c}\text { tocopherol+h } \\
\text { ydration }\end{array}$ & $11 / 204$ & $\begin{array}{c}\text { placebo+hy } \\
\text { dration }\end{array}$ & $15 / 101$ & & & Non-industry \\
\hline Stephen ${ }^{129}$ & 2013 & 357 & 41 & 61 & \begin{tabular}{|c|} 
iodixanol \\
or \\
iopamidol \\
or ioversol
\end{tabular} & $\begin{array}{l}\text { low- or } \\
\text { iso- } \\
\text { osmolar }\end{array}$ & 123 & $\mathrm{Scr}>1.4 \mathrm{mg} / \mathrm{dl}$ & $\begin{array}{c}\text { NAC+hydrati } \\
\text { on }\end{array}$ & $14 / 185$ & $\begin{array}{c}\text { placebo+hy } \\
\text { dration }\end{array}$ & $12 / 172$ & & & Non-industry \\
\hline
\end{tabular}




\begin{tabular}{|c|c|c|c|c|c|c|c|c|c|c|c|c|c|c|c|c|c|}
\hline Erturk $^{130}$ & 2014 & 307 & 64 & 66 & iopromide & $\begin{array}{c}\text { low- } \\
\text { osmolar }\end{array}$ & 126 & $\begin{array}{c}\text { eGFR }<60 \mathrm{ml} / \\
\min \end{array}$ & $\begin{array}{c}\text { NAC+hydrati } \\
\text { on }\end{array}$ & 27/204 & $\begin{array}{c}\text { placebo+hy } \\
\text { dration }\end{array}$ & $7 / 103$ & & & & & $\begin{array}{l}\text { No funding } \\
\text { supported }\end{array}$ \\
\hline $\operatorname{Han}^{131}$ & 2014 & 2998 & 39 & 61 & iodixanol & $\begin{array}{l}\text { iso- } \\
\text { osmolar }\end{array}$ & 115 & $\begin{array}{c}\text { CKD stage 2- } \\
3 \text { and DM }\end{array}$ & $\begin{array}{c}\text { rosuvastatin1 } \\
\text { Omg+hydratio } \\
\mathrm{n}\end{array}$ & $34 / 1498$ & hydration & $58 / 1500$ & & & & & NA \\
\hline Antonto $^{132}$ & 2014 & 500 & 61 & 59 & $\begin{array}{c}\text { ioxitalama } \\
\text { te }\end{array}$ & $\begin{array}{l}\text { high- } \\
\text { osmolar }\end{array}$ & 91 & $\begin{array}{l}\text { no limited } \\
\text { kidney } \\
\text { function }\end{array}$ & $\begin{array}{c}\text { NAC+hydrati } \\
\text { on }\end{array}$ & $49 / 126$ & $\begin{array}{c}\mathrm{NaHCO}_{3}+\mathrm{h} \\
\text { ydration }\end{array}$ & $75 / 125$ & $\left|\begin{array}{c}\mathrm{NaHCO}_{3} \\
+\mathrm{NAC}\end{array}\right|$ & $72 / 124$ & hydration & $61 / 125$ & Non-industry \\
\hline Kama ${ }^{133}$ & 2014 & 107 & 55 & 71 & iohexol & $\begin{array}{l}\text { low- } \\
\text { osmolar }\end{array}$ & NA & $\begin{array}{l}\text { no limited } \\
\text { kidney } \\
\text { function }\end{array}$ & $\begin{array}{c}\text { NAC+hydrati } \\
\text { on }\end{array}$ & $7 / 36$ & $\begin{array}{c}\mathrm{NaHCO}_{3}+\mathrm{h} \\
\text { ydration }\end{array}$ & $4 / 36$ & $\begin{array}{c}\text { hydratio } \\
\mathrm{n}\end{array}$ & $5 / 35$ & & & $\begin{array}{l}\text { No funding } \\
\text { supported }\end{array}$ \\
\hline $\begin{array}{l}\text { PRATO- } \\
\text { ACS } 134\end{array}$ & 2014 & 504 & 66 & 66 & iodixanol & $\begin{array}{l}\text { iso- } \\
\text { osmolar }\end{array}$ & 144 & no dialysis & $\begin{array}{c}\text { rosuvastatin2 } \\
0-40 \mathrm{mg} \\
\text { +hydration+ } \\
\text { NAC }\end{array}$ & $17 / 252$ & $\begin{array}{c}\text { NAC+hydra } \\
\text { tion }\end{array}$ & $38 / 252$ & & & & & Non-industry \\
\hline $\mathrm{Li}^{135}$ & 2014 & 163 & 67 & 64 & iohexol & $\begin{array}{c}\text { low- } \\
\text { osmolar }\end{array}$ & 170 & $\begin{array}{c}\text { eGFR } \geq 30 \mathrm{ml} / \\
\min \end{array}$ & $\begin{array}{l}\text { Prostaglandin } \\
\text { E1+hydration }\end{array}$ & $3 / 82$ & hydration & 9/81 & & & & & Non-industry \\
\hline Manari'136 & 2014 & 592 & 75 & 65 & iodixanol & $\begin{array}{c}\text { iso- } \\
\text { osmolar }\end{array}$ & 198 & no dialysis & $\mathrm{NaHCO}_{3}$ & $56 / 293$ & hydration & $51 / 299$ & & & & & NA \\
\hline PROMEC $^{13}$ & 2014 & 231 & 55 & 60 & iohexol & $\begin{array}{c}\text { low- } \\
\text { osmolar }\end{array}$ & 100 & $\mathrm{Scr}>1.2 \mathrm{mg} / \mathrm{dl}$ & $\mathrm{NaHCO}_{3}$ & $12 / 111$ & hydration & $8 / 120$ & & & & & $\begin{array}{l}\text { No funding } \\
\text { supported }\end{array}$ \\
\hline Thayssen $^{13}$ & 2014 & 715 & 77 & 63 & iodixanol & $\begin{array}{c}\text { iso- } \\
\text { osmolar }\end{array}$ & 140 & no dialysis & hydration & 43/181 & $\begin{array}{c}\text { NAC+hydra } \\
\text { tion }\end{array}$ & $32 / 176$ & $\begin{array}{c}\mathrm{NaHCO}_{3} \\
\text { +hydrati } \\
\text { on }\end{array}$ & $33 / 181$ & $\left|\begin{array}{c}\mathrm{NaHCO}_{3} \\
+\mathrm{NAC}\end{array}\right|$ & $33 / 177$ & Non-industry \\
\hline Yang ${ }^{139}$ & 2014 & 527 & 45 & 59 & iohexol & $\begin{array}{c}\text { low- } \\
\text { osmolar }\end{array}$ & 127 & $\begin{array}{c}\text { eGFR } \geq 30 \mathrm{ml} / \\
\min \end{array}$ & hydration & $5 / 161$ & $\begin{array}{c}\text { NAC+hydra } \\
\text { tion }\end{array}$ & $7 / 157$ & $\mathrm{NaHCO}_{3} \mid$ & $8 / 159$ & $\begin{array}{c}\mathrm{NaHCO}_{3} \\
+\mathrm{NAC}\end{array}$ & $8 / 150$ & $\begin{array}{l}\text { No funding } \\
\text { supported }\end{array}$ \\
\hline Fahmy ${ }^{140}$ & 2014 & 200 & NA & NA & NA & NA & NA & NA & $\begin{array}{c}\text { Rosuvastatin } \\
20 \mathrm{mg}+\text { hydrati } \\
\text { on }\end{array}$ & $15 / 100$ & $\begin{array}{c}\text { placebo+hy } \\
\text { dration }\end{array}$ & $38 / 100$ & & & & & NA \\
\hline \begin{tabular}{|l|} 
Yeganehkh \\
ah141
\end{tabular} & 2014 & 78 & 78 & 59 & iohexol & $\begin{array}{c}\text { low- } \\
\text { osmolar }\end{array}$ & 44 & Scr $\leq 4 \mathrm{mg} / \mathrm{dl}$ & $\begin{array}{c}\mathrm{NaHCO} 3+h y \\
\text { dration }\end{array}$ & $7 / 50$ & $\begin{array}{c}\text { NAC+hydra } \\
\text { tion }\end{array}$ & $6 / 50$ & $\begin{array}{c}\text { hydratio } \\
\mathrm{n}\end{array}$ & $7 / 50$ & & & Non-industry \\
\hline Abaci ${ }^{142}$ & 2015 & 208 & 71 & 68 & ioversol & $\begin{array}{c}\text { low- } \\
\text { osmolar }\end{array}$ & 129 & $\begin{array}{l}\text { eGFR 30- } \\
60 \mathrm{ml} / \mathrm{min}\end{array}$ & $\begin{array}{c}\text { Rosuvastatin } \\
40 \mathrm{mg}+\text { hydrati } \\
\text { on }\end{array}$ & $6 / 103$ & hydration & 9/105 & & & & & $\begin{array}{l}\text { No funding } \\
\text { supported }\end{array}$ \\
\hline $\begin{array}{l}\text { Arabmome } \\
\text { ni143 }\end{array}$ & 2015 & 62 & 43 & 62 & iodixanol & $\begin{array}{c}\text { low- } \\
\text { osmolar }\end{array}$ & 136 & $\begin{array}{c}\text { normal kidney } \\
\text { function }\end{array}$ & $\begin{array}{c}\text { theophylline+ } \\
\text { hydration }\end{array}$ & $6 / 30$ & $\begin{array}{c}\text { NAC+hydra } \\
\text { tion }\end{array}$ & $7 / 32$ & & & & & Non-industry \\
\hline Bidram ${ }^{144}$ & 2015 & 200 & 91 & 60 & iodixanol & $\begin{array}{l}\text { iso- } \\
\text { osmolar }\end{array}$ & 35 & $\begin{array}{c}\text { eGFR } \geq 60 \mathrm{ml} / \\
\mathrm{min}\end{array}$ & $\begin{array}{c}\text { atorvastatin } \\
80 \mathrm{mg}+\text { hydrati } \\
\text { on }\end{array}$ & $1 / 100$ & $\begin{array}{c}\text { placebo+hy } \\
\text { dration }\end{array}$ & $2 / 100$ & & & & & NA \\
\hline $\begin{array}{l}\text { CONTRAS } \\
T^{145}\end{array}$ & 2015 & 453 & 76 & 68 & \begin{tabular}{|c|} 
iohexol or \\
iopamidol \\
or ioversol \\
or \\
iopromide \\
\end{tabular} & $\begin{array}{l}\text { low- } \\
\text { osmolar }\end{array}$ & 116 & $\begin{array}{l}\text { eGFR 15- } \\
60 \mathrm{ml} / \mathrm{min}\end{array}$ & $\begin{array}{c}\text { NAC+hydrati } \\
\text { on }\end{array}$ & 10/153 & $\mathrm{NaHCO} 3$ & 19/149 & $\mid \begin{array}{c}\mathrm{NAC}+\mathrm{Na} \\
\mathrm{HCO} 3\end{array}$ & 16/151 & & & $\begin{array}{l}\text { No funding } \\
\text { supported }\end{array}$ \\
\hline Galal ${ }^{146}$ & 2015 & 80 & 64 & 56 & NA & $\begin{array}{c}\text { low- } \\
\text { osmolar }\end{array}$ & 241 & $\begin{array}{l}\text { eGFR 60- } \\
90 \mathrm{ml} / \mathrm{min}\end{array}$ & \begin{tabular}{|c|} 
Atorvastatin8 \\
Omg+hydratio \\
$\mathrm{n}$
\end{tabular} & $5 / 40$ & $\begin{array}{c}\text { Atorvastati } \\
\text { n10mg+hy } \\
\text { dration }\end{array}$ & $7 / 40$ & & & & & $\begin{array}{l}\text { No funding } \\
\text { supported }\end{array}$ \\
\hline
\end{tabular}




\begin{tabular}{|c|c|c|c|c|c|c|c|c|c|c|c|c|c|}
\hline Jo & 2015 & 218 & 85 & 59 & NA & NA & NA & $\begin{array}{l}\text { no limited } \\
\text { kidney } \\
\text { function }\end{array}$ & \begin{tabular}{|c|} 
Atorvastatin8 \\
Omg+hydratio \\
$n$ \\
\end{tabular} & $6 / 110$ & $\begin{array}{c}\text { Atorvastati } \\
\text { n10mg+hy } \\
\text { dration }\end{array}$ & 11/108 & Non-industry \\
\hline Shehata $^{148}$ & 2015 & 130 & 62 & 56 & iopromide & $\begin{array}{c}\text { low- } \\
\text { osmolar }\end{array}$ & 276 & $\begin{array}{l}\text { eGFR 30- } \\
90 \mathrm{ml} / \mathrm{min}\end{array}$ & $\begin{array}{c}\text { atorvastatin } \\
\text { 80mg+hydrati } \\
\text { on+NAC }\end{array}$ & $5 / 65$ & $\begin{array}{c}\text { NAC+hydra } \\
\text { tion }\end{array}$ & $13 / 65$ & $\begin{array}{l}\text { No funding } \\
\text { supported }\end{array}$ \\
\hline BOSS $^{149}$ & 2015 & 391 & 58 & 72 & NA & NA & 107 & $\begin{array}{c}\text { eGFR }<45 \mathrm{ml} / \\
\min \end{array}$ & $\mathrm{NaHCO} 3$ & 26/195 & hydration & $18 / 196$ & Non-industry \\
\hline Rezaei150 & 2016 & 298 & 69 & 67 & iodixanol & $\begin{array}{c}\text { low- } \\
\text { osmolar }\end{array}$ & 50 & $\begin{array}{c}\text { eGFR }<60 \mathrm{ml} / \\
\mathrm{min}\end{array}$ & $\begin{array}{c}\text { Vitamin } \\
\text { E+hydration }\end{array}$ & $10 / 149$ & $\begin{array}{c}\text { placebo+hy } \\
\text { dration }\end{array}$ & $21 / 149$ & Non-industry \\
\hline
\end{tabular}

ALA, alpha-lipoic acid; ANP, atrial natriuretic peptide; BNP, B-type natriuretic peptide; CI-AKI, contrast-induced acute kidney injury; Crcl, Creatinine clearance rate; eGFR, estimated glomerular filtration rate; NA, no available; NAC, $\mathrm{N}$-acetylcysteine; $\mathrm{NaHCO}_{3}$, bicarbonate sodium; Scr, Serum creatinine. 


\section{Reference}

1. Gurkowski L, MacDougall M, Wiegmann T. Effects of Misoprostol on Contrast-Induced Renal Dysfunction. Am J Ther. 1995; 2: 837-842.

2. Kurnik BR, Allgren RL, Genter FC, Solomon RJ, Bates ER, Weisberg LS. Prospective study of atrial natriuretic peptide for the prevention of radiocontrast-induced nephropathy. Am J Kidney Dis. 1998; 31: 674-680.

3. Abizaid AS, Clark CE, Mintz GS, et al. Effects of dopamine and aminophylline on contrast-induced acute renal failure after coronary angioplasty in patients with preexisting renal insufficiency. Am J Cardiol. 1999; 83: 260-263, A5.

4. Erley CM, Duda SH, Rehfuss D, et al. Prevention of radiocontrast-media-induced nephropathy in patients with preexisting renal insufficiency by hydration in combination with the adenosine antagonist theophylline. Nephrol Dial Transplant. 1999; 14: 1146-1149.

5. Koch JA, Plum J, Grabensee B, Modder U. Prostaglandin E1: A new agent for the prevention of renal dysfunction in high risk patients caused by radiocontrast media? Nephrol Dial Transplant. 2000; 15: 43-49.

6. Tepel M, van der Giet M, Schwarzfeld C, Laufer U, Liermann D, Zidek W. Prevention of radiographic-contrast-agentinduced reductions in renal function by acetylcysteine. N Engl J Med. 2000; 343: 180-184.

7. Allaqaband S, Tumuluri R, Malik AM, et al. Prospective randomized study of N-acetylcysteine, fenoldopam, and saline for prevention of radiocontrast-induced nephropathy. Catheter Cardiovasc Interv. 2002; 57: 279-283.

8. Briguori C, Manganelli F, Scarpato P, et al. Acetylcysteine and contrast agent-associated nephrotoxicity. J Am Coll Cardiol. 2002; 40: 298-303.

9. Diaz-Sandoval LJ, Kosowsky BD, Losordo DW. Acetylcysteine to prevent angiography-related renal tissue injury (the APART trial). Am J Cardiol. 2002; 89: 356-358.

10. Durham JD, Caputo C, Dokko J, et al. A randomized controlled trial of N-acetylcysteine to prevent contrast nephropathy in cardiac angiography. Kidney Int. 2002; 62: 2202-2207.

11. Huber W, Ilgmann K, Page M, et al. Effect of theophylline on contrast material-induced nephropathy in patients with chronic renal insufficiency: Controlled, randomized, double-blinded study. Radiology. 2002; 223: 772-779. 
12. Kramer BK, Preuner J, Ebenburger A, et al. Lack of renoprotective effect of theophylline during aortocoronary bypass surgery. Nephrol Dial Transplant. 2002; 17: 910-915.

13. Shyu KG, Cheng JJ, Kuan P. Acetylcysteine protects against acute renal damage in patients with abnormal renal function undergoing a coronary procedure. J Am Coll Cardiol. 2002; 40:1383-1388.

14. Tumlin JA, Wang A, Murray PT, Mathur VS. Fenoldopam mesylate blocks reductions in renal plasma flow after radiocontrast dye infusion: a pilot trial in the prevention of contrast nephropathy. Am Heart J. 2002; 143: 894-903.

15. Baker CS, Wragg A, Kumar S, De Palma R, Baker LR, Knight CJ. A rapid protocol for the prevention of contrast-induced renal dysfunction: the RAPPID study. J Am Coll Cardiol. 2003; 41: 2114-2118.

16. Boccalandro F, Amhad M, Smalling RW, Sdringola S. Oral acetylcysteine does not protect renal function from moderate to high doses of intravenous radiographic contrast. Catheter Cardiovasc Interv. 2003; 58(3): 336-341.

17. Efrati S, Dishy V,Averbukh M, et al. The effect of N-acetylcysteine on renal function, nitric oxide, and oxidative stress after angiography. Kidney Int. 2003; 64(6): 2182-2187.

18. El Mahmoud R, Le Feuvre C, Le Quan Sang KH, et al. Absence of nephro-protective effect of acetylcysteine in patients with chronic renal failure investigated by coronary angiography. Arch Mal Coeur Vaiss. 2003; 96: 1157-1161.

19. Huber W, Schipek C, Ilgmann K, et al. Effectiveness of theophylline prophylaxis of renal impairment after coronary angiography in patients with chronic renal insufficiency. Am J Cardiol. 2003; 91: 1157-1162.

20. Kay J, Chow WH, Chan TM, et al. Acetylcysteine for prevention of acute deterioration of renal function following elective coronary angiography and intervention: a randomized controlled trial. JAMA. 2003; 289: 553-558.

21. Kefer JM, Hanet CE, Boitte S, Wilmotte L, De Kock M. Acetylcysteine, coronary procedure and prevention of contrastinduced worsening of renal function: which benefit for which patient? Acta Cardiol. 2003; 58:555-560.

22. MacNeill BD, Harding SA, Bazari H, et al. Prophylaxis of contrast-induced nephropathy in patients undergoing coronary angiography. Catheter Cardiovasc Interv. 2003; 60: 458-461.

23. Oldemeyer JB, Biddle WP, Wurdeman RL, Mooss AN, Cichowski E, Hilleman DE. Acetylcysteine in the prevention of contrast-induced nephropathy after coronary angiography. Am Heart J. 2003; 146: E23. 
24. Stone GW, McCullough PA, Tumlin JA, et al. Fenoldopam mesylate for the prevention of contrast-induced nephropathy: a randomized controlled trial. JAMA. 2003; 290: 2284-2291.

25. Agrawal M, Wodlinger AM, Huggins CE, et al. Effect of N-acetylcysteine on serum creatinine concentration in patients with chronic renal insufficiency who are undergoing coronary angiography. Heart Drug. 2004; 4: 87-91.

26. Balderramo DC, Verdu MB, Ramacciotti CF, et al. Renoprotective effect of high periprocedural doses of oral Nacetylcysteine in patients scheduled to undergo a same-day angiography. Revista de la Facultad de Ciencias Medicas de Cordoba. 2004; 61: 13-19.

27. Briguori C, Colombo A, Airoldi F, et al. N-Acetylcysteine versus fenoldopam mesylate to prevent contrast agentassociated nephrotoxicity. J Am Coll Cardiol. 2004; 44: 762-765.

28. Fung JW, Szeto CC, Chan WW, et al. Effect of N-acetylcysteine for prevention of contrast nephropathy in patients with moderate to severe renal insufficiency: a randomized trial. Am J Kidney Dis. 2004; 43: 801-808.

29. Goldenberg I, Shechter M, Matetzky S, et al. Oral acetylcysteine as an adjunct to saline hydration for the prevention of contrast-induced nephropathy following coronary angiography. A randomized controlled trial and review of the current literature. Eur Heart J. 2004; 25: 212-218.

30. Merten GJ, Burgess WP, Gray LV, et al. Prevention of contrast-induced nephropathy with sodium bicarbonate: a randomized controlled trial. JAMA. 2004; 291: 2328-2334.

31. Miner SE, Dzavik V, Nguyen-Ho P, et al. N-acetylcysteine reduces contrast-associated nephropathy but not clinical events during long-term follow-up. Am Heart J. 2004; 148: 690-695.

32. Ochoa A, Pellizzon G, Addala S, et al. Abbreviated dosing of N-acetylcysteine prevents contrast-induced nephropathy after elective and urgent coronary angiography and intervention. J Interv Cardiol. 2004; 17:159-165.

33. Rashid ST, Salman M, Myint F, et al. Prevention of contrast-induced nephropathy in vascular patients undergoing angiography: a randomized controlled trial of intravenous N-acetylcysteine. J Vasc Surg. 2004; 40: 1136-1141.

34. Spargias K, Alexopoulos E, Kyrzopoulos S, et al. Ascorbic acid prevents contrast-mediated nephropathy in patients with renal dysfunction undergoing coronary angiography or intervention. Circulation. 2004; 110: 2837-2842. 
35. Webb JG, Pate GE, Humphries $\mathrm{KH}$, et al. A randomized controlled trial of intravenous N-acetylcysteine for the prevention of contrast-induced nephropathy after cardiac catheterization: lack of effect. Am Heart J. 2004; 148:422-429.

36. Azmus AD, Gottschall C, Manica A, et al. Effectiveness of acetylcysteine in prevention of contrast nephropathy. $J$ Invasive Cardiol. 2005; 17: 80-84.

37. Gomes VO, Poli de Figueredo CE, Caramori P, et al. N-acetylcysteine does not prevent contrast induced nephropathy after cardiac catheterisation with an ionic low osmolality contrast medium: a multicentre clinical trial. Heart. 2005; 91: 774778.

38. Gulel O, Keles T, Eraslan H, Aydogdu S, Diker E, Ulusoy V. Prophylactic acetylcysteine usage for prevention of contrast nephropathy after coronary angiography. J Cardiovasc Pharmacol. 2005; 46:464-467.

39. Kotlyar E, Keogh AM, Thavapalachandran S, et al. Prehydration alone is sufficient to prevent contrast-induced nephropathy after day-only angiography procedures - A randomised controlled trial. Heart Lung and Circulation. 2005; 14(4): $245-251$.

40. Coyle LC, Rodriguez A, Jeschke RE, Simon-Lee A, Abbott KC, Taylor AJ. Acetylcysteine In Diabetes (AID): a randomized study of acetylcysteine for the prevention of contrast nephropathy in diabetics. Am Heart J. 2006; 151: 1032.e912.

41. Dussol B, Morange S, Loundoun A, Auquier P, Berland Y. A randomized trial of saline hydration to prevent contrast nephropathy in chronic renal failure patients. Nephrol Dial Transplant. 2006; 21: 2120-2126.

42. Khalili H, Dashti-Khavidaki S, Tabifar H, Ahmadinejad N, Ahmadi F. N-acetylcysteine in the prevention of contrast agent-induced nephrotoxicity in patients undergoing computed tomography studies. Therapy. 2006; 3: 773-777.

43. Marenzi G, Assanelli E, Marana I, et al. N-acetylcysteine and contrast-induced nephropathy in primary angioplasty. $N$ Engl J Med. 2006; 354: 2773-2782.

44. Ng TM, Shurmur SW, Silver M, et al. Comparison of N-acetylcysteine and fenoldopam for preventing contrast-induced nephropathy (CAFCIN). Int J Cardiol. 2006; 109: 322-328.

45. Boscheri A, Weinbrenner C, Botzek B, Reynen K, Kuhlisch E, Strasser RH. Failure of ascorbic acid to prevent contrast- 
media induced nephropathy in patients with renal dysfunction. Clin Nephrol. 2007; 68: 279-286.

46. Briguori C, Airoldi F, D'Andrea D, et al. Renal Insufficiency Following Contrast Media Administration Trial (REMEDIAL): a randomized comparison of 3 preventive strategies. Circulation. 2007; 115: 1211-1217.

47. Carbonell N, Blasco M, Sanjuan R, et al. Intravenous N-acetylcysteine for preventing contrast-induced nephropathy: a randomised trial. Int J Cardiol. 2007; 115: 57-62.

48. Hobikoglu GF, Norgaz T, Aksu H, et al. The effectiveness of oral N-acetylcysteine administered three hours before angiography in the prevention of contrast nephropathy in patients with mildly elevated creatinine levels. Turk Kardiyoloji Dernegi arsivi. 2007; 35: 9-12.

49. Hsu CH, Lee JD, Lo PH, Lin JJ, Chang HW, Chou HT. Prevention of radiocontrast-induced nephropathy with Nacetylcysteine after cardiac angiography in diabetic patients with renal dysfunction. Mid-Taiwan Journal of Medicine. 2007;

12: 173-183.

50. Lawlor DK, Moist L, DeRose G, et al. Prevention of contrast-induced nephropathy in vascular surgery patients. Ann Vasc Surg. 2007; 21: 593-597.

51. Masuda M, Yamada T, Mine T, et al. Comparison of usefulness of sodium bicarbonate versus sodium chloride to prevent contrast-induced nephropathy in patients undergoing an emergent coronary procedure. Am J Cardiol. 2007; 100:781-786.

52. Ozcan EE, Guneri S, Akdeniz B, et al. Sodium bicarbonate, N-acetylcysteine, and saline for prevention of radiocontrastinduced nephropathy. A comparison of 3 regimens for protecting contrast-induced nephropathy in patients undergoing coronary procedures. A single-center prospective controlled trial. Am Heart J. 2007; 154: 539-544.

53. Recio-Mayoral A, Chaparro M, Prado B, et al. The reno-protective effect of hydration with sodium bicarbonate plus Nacetylcysteine in patients undergoing emergency percutaneous coronary intervention: the RENO Study. $J$ Am Coll Cardiol. 2007; 49: 1283-1288.

54. Reinecke H, Fobker M, Wellmann J, et al. A randomized controlled trial comparing hydration therapy to additional hemodialysis or N-acetylcysteine for the prevention of contrast medium-induced nephropathy: the Dialysis-versus-Diuresis (DVD) Trial. Clin Res Cardiol. 2007; 96(3):130-139. 
55. Seyon RA, Jensen LA, Ferguson IA, Williams RG. Efficacy of N-acetylcysteine and hydration versus placebo and hydration in decreasing contrast-induced renal dysfunction in patients undergoing coronary angiography with or without concomitant percutaneous coronary intervention. Heart Lung. 2007; 36: 195-204.

56. Adolph E, Holdt-Lehmann B, Chatterjee T, et al. Renal Insufficiency Following Radiocontrast Exposure Trial (REINFORCE): a randomized comparison of sodium bicarbonate versus sodium chloride hydration for the prevention of contrast-induced nephropathy. Coron Artery Dis. 2008; 19: 413-419.

57. Brar SS, Shen AY, Jorgensen MB, et al. Sodium bicarbonate vs sodium chloride for the prevention of contrast mediuminduced nephropathy in patients undergoing coronary angiography: a randomized trial. JAMA. 2008; 300: 1038-1046.

58. Heng AE, Cellarier E, Aublet-Cuvelier B, et al. Is treatment with N-acetylcysteine to prevent contrast-induced nephropathy when using bicarbonate hydration out of date? Clin Nephrol. 2008; 70:475-484.

59. Izani Wan Mohamed WM, Darus Z, Yusof Z. Oral N-acetylcysteine in prevention of contrast induced nephropathy following coronary angiogram. Int Med J. 2008; 15: 353-361.

60. Jo SH, Koo BK, Park JS, et al. Prevention of radiocontrast medium-induced nephropathy using short-term high-dose simvastatin in patients with renal insufficiency undergoing coronary angiography (PROMISS) trial-a randomized controlled study. Am Heart J. 2008; 155: 499.e1-e8.

61. Kimmel M, Butscheid M, Brenner S, Kuhlmann U, Klotz U, Alscher DM. Improved estimation of glomerular filtration rate by serum cystatin C in preventing contrast induced nephropathy by N-acetylcysteine or zinc--preliminary results. Nephrol Dial Transplant. 2008; 23: 1241-1245.

62. Maioli M, Toso A, Leoncini M, et al. Sodium bicarbonate versus saline for the prevention of contrast-induced nephropathy in patients with renal dysfunction undergoing coronary angiography or intervention. J Am Coll Cardiol. 2008; 52: 599-604.

63. Masuda M, Yamada T, Okuyama Y, et al. Sodium bicarbonate improves long-term clinical outcomes compared with sodium chloride in patients with chronic kidney disease undergoing an emergent coronary procedure. Circ J. 2008; 72: 16101614. 
64. Wang JH, Subeq YM, Tsai WC, Lee RP, Hsu BG. Intravenous N-acetylcysteine with saline hydration improves renal function and ameliorates plasma total homocysteine in patients undergoing cardiac angiography. Ren Fail. 2008; 30: 527-533.

65. Amini M, Salarifar M, Amirbaigloo A, Masoudkabir F, Esfahani F. N-acetylcysteine does not prevent contrast-induced nephropathy after cardiac catheterization in patients with diabetes mellitus and chronic kidney disease: a randomized clinical trial. Trials. 2009; 10: 45.

66. Baskurt M, Okcun B, Abaci O, et al. N-acetylcysteine versus N-acetylcysteine + theophylline for the prevention of contrast nephropathy. Eur J Clin Invest. 2009; 39: 793-799.

67. Ferrario F, Barone MT, Landoni G, et al. Acetylcysteine and non-ionic isosmolar contrast-induced nephropathy - A randomized controlled study. Nephrol Dial Transplant. 2009; 24: 3103-3107.

68. Kim HS, Lee JH, Kim MG, et al. Evaluation of alpha lipoic acid to prevent contrast-induced nephropathy: A prospective randomized trial. Am J Cardiol. 2009; 103: 108B.

69. Jo SH, Koo BK, Park JS, et al. N-acetylcysteine versus AScorbic acid for preventing contrast-Induced nephropathy in patients with renal insufficiency undergoing coronary angiography NASPI study-a prospective randomized controlled trial. Am Heart J. 2009; 157: 576-583.

70. Morikawa S, Sone T, Tsuboi H, et al. Renal protective effects and the prevention of contrast-induced nephropathy by atrial natriuretic peptide. J Am Coll Cardiol. 2009; 53:1040-1046.

71. Pakfetrat M, Nikoo MH, Malekmakan L, et al. A comparison of sodium bicarbonate infusion versus normal saline infusion and its combination with oral acetazolamide for prevention of contrast-induced nephropathy: a randomized, doubleblind trial. Int Urol Nephrol. 2009; 41:629-634.

72. Ratcliffe JA, Thiagarajah P, Chen J, et al. Prevention of contrast-induced nephropathy: A randomized controlled trial of sodium bicarbonate and N-acetylcysteine. Int J Angiol. 2009; 18: 193-197.

73. Spargias K, Adreanides E, Demerouti E, et al. Iloprost prevents contrast-induced nephropathy in patients with renal dysfunction undergoing coronary angiography or intervention. Circulation. 2009; 120:1793-1799.

74. Tamura A, Goto Y, Miyamoto K, et al. Efficacy of single-bolus administration of sodium bicarbonate to prevent contrast- 
induced nephropathy in patients with mild renal insufficiency undergoing an elective coronary procedure. Am J Cardiol. 2009;

104: 921-925.

75. Tasanarong A, Piyayotai D, Thitiarchakul S. Protection of radiocontrast induced nephropathy by vitamin E (alpha tocopherol): a randomized controlled pilot study. J Med Assoc Thai. 2009; 92: 1273-1281.

76. Vasheghani-Farahani A, Sadigh G, Kassaian SE, et al. Sodium bicarbonate plus isotonic saline versus saline for prevention of contrast-induced nephropathy in patients undergoing coronary angiography: a randomized controlled trial. Am J Kidney Dis. 2009; 54: 610-618.

77. Xinwei J, Xianghua F, Jing Z, et al. Comparison of usefulness of simvastatin $20 \mathrm{mg}$ versus $80 \mathrm{mg}$ in preventing contrastinduced nephropathy in patients with acute coronary syndrome undergoing percutaneous coronary intervention. Am J Cardiol. 2009; 104: 519-524.

78. Zhou X, Jin YZ, Wang Q, Min R, Zhang XY. Efficacy of high dose atorvastatin on preventing contrast induced nephropathy in patients underwent coronary angiography. Chin J Cardiol. 2009; 37: 394-396.

79. Buyukhatipoglu H, Sezen Y, Yildiz A, et al. N-acetylcysteine fails to prevent renal dysfunction and oxidative stress after noniodine contrast media administration during percutaneous coronary interventions. Pol Arch Med Wewn. 2010; 120: 383389.

80. Carbonell N, Sanjuan R, Blasco M, Jorda A, Miguel A. N-acetylcysteine: Short-Term Clinical Benefits After Coronary Angiography in High-Risk Renal Patients. Rev Esp Cardiol. 2010; 63: 12-19.

81. Castini D, Lucreziotti S, Bosotti L, et al. Prevention of contrast-induced nephropathy: a single center randomized study. Clin Cardiol. 2010; 33: E63-68.

82. Kim BJ, Sung KC, Kim BS, et al. Effect of N-acetylcysteine on cystatin C-based renal function after elective coronary angiography (ENABLE Study): a prospective, randomized trial. Int J Cardiol. 2010; 138:239-245.

83. Kinbara T, Hayano T, Ohtani N, Furutani Y, Moritani K, Matsuzaki M. Efficacy of N-acetylcysteine and aminophylline in preventing contrast-induced nephropathy. J Cardiol. 2010; 55: 174-179.

84. Malhis M, Al-Bitar S, Al-Deen Zaiat K. The role of theophylline in prevention of radiocontrast media-induced 
nephropathy. Saudi J Kidney Dis Transpl. 2010; 21: 276-283.

85. Matejka J, Varvarovsky I, Vojtisek P, et al. Prevention of contrast-induced acute kidney injury by theophylline in elderly patients with chronic kidney disease. Heart Vessels. 2010; 25: 536-542.

86. Ozhan H, Erden I, Ordu S, et al. Efficacy of short-term high-dose atorvastatin for prevention of contrast-induced nephropathy in patients undergoing coronary angiography. Angiology. 2010; 61: 711-714.

87. Rohani A. Effectiveness of aminophylline prophylaxis of renal impairment after coronary angiography in patients with chronic renal insufficiency. Indian J Nephrol. 2010; 20: 80-83.

88. Sar F, Saler T, Ecebay A, Saglam ZA, Ozturk S, Kazancioglu R. The efficacy of n-acetylcysteine in preventing contrastinduced nephropathy in type 2 diabetic patients without nephropathy. J Nephrol. 2010; 23: 478-482.

89. Thiele H, Hildebrand L, Schirdewahn C, et al. Impact of High-Dose N-Acetylcysteine Versus Placebo on ContrastInduced Nephropathy and Myocardial Reperfusion Injury in Unselected Patients With ST-Segment Elevation Myocardial Infarction Undergoing Primary Percutaneous Coronary Intervention. J Am Coll Cardiol. 2010; 55: 2201-2209.

90. Toso A, Maioli M, Leoncini M, et al. Usefulness of atorvastatin (80 mg) in prevention of contrast-induced nephropathy in patients with chronic renal disease. Am J Cardiol. 2010; 105: 288-292.

91. Vasheghani-Farahani A, Sadigh G, Kassaian SE, et al. Sodium bicarbonate in preventing contrast nephropathy in patients at risk for volume overload: a randomized controlled trial. J Nephrol. 2010; 23:216-223.

92. Zhang J, Fu X, Jia X, et al. B-type natriuretic peptide for prevention of contrast-induced nephropathy in patients with heart failure undergoing primary percutaneous coronary intervention. Acta Radiol. 2010; 51:641-648.

93. Investigators A. Acetylcysteine for prevention of renal outcomes in patients undergoing coronary and peripheral vascular angiography: main results from the randomized Acetylcysteine for Contrast-induced nephropathy Trial (ACT). Circulation. 2011; 124: 1250-1259.

94. Kim JW, Ha SJ, Kim W, et al. Effect of single high-dose atorvastatin pretreatment to prevent contrast-induced nephropathy in patients undergoing percutaneous coronary intervention. Am J Cardiol. 2011; 107: 102A.

95. Lee SW, Kim WJ, Kim YH, et al. Preventive strategies of renal insufficiency in patients with diabetes undergoing 
intervention or arteriography (the PREVENT Trial). Am J Cardiol. 2011; 107: 1447-1452.

96. Maioli M, Toso A, Leoncini M, Micheletti C, Bellandi F. Effects of hydration in contrast-induced acute kidney injury after primary angioplasty: a randomized, controlled trial. Circ Cardiovasc Interv. 2011; 4: 456-462.

97. Motohiro M, Kamihata H, Tsujimoto S, et al. A new protocol using sodium bicarbonate for the prevention of contrastinduced nephropathy in patients undergoing coronary angiography. Am J Cardiol. 2011; 107:1604-1608.

98. Patti G, Ricottini E, Nusca A, et al. Short-term, high-dose Atorvastatin pretreatment to prevent contrast-induced nephropathy in patients with acute coronary syndromes undergoing percutaneous coronary intervention (from the ARMYDACIN [atorvastatin for reduction of myocardial damage during angioplasty--contrast-induced nephropathy] trial). Am J Cardiol. 2011; 108: 1-7.

99. Sadat U, Walsh SR, Norden AG, Gillard JH, Boyle JR. Does oral N-acetylcysteine reduce contrast-induced renal injury in patients with peripheral arterial disease undergoing peripheral angiography? A randomized-controlled study. Angiology. 2011; 62: 225-230.

100. Tanaka A, Suzuki Y, Suzuki N, et al. Does N-acetylcysteine reduce the incidence of contrast-induced nephropathy and clinical events in patients undergoing primary angioplasty for acute myocardial infarction? Intern Med. 2011; 50:673-677.

101. 145 Ueda H, Yamada T, Masuda M, et al. Prevention of contrast-induced nephropathy by bolus injection of sodium bicarbonate in patients with chronic kidney disease undergoing emergent coronary procedures. Am J Cardiol. 2011; 107: 1163-1167.

102. Aslanger E, Uslu B, Akdeniz C, Polat N, Cizgici Y, Oflaz H. Intrarenal application of N-acetylcysteine for the prevention of contrast medium-induced nephropathy in primary angioplasty. Coron Artery Dis. 2012; 23: 265-270.

103. Bilasy ME, Oraby MA, Ismail HM, Maklady FA. Effectiveness of theophylline in preventing contrast-induced nephropathy after coronary angiographic procedures. J Interv Cardiol. 2012; 25: 404-410.

104. Cao S, Wang P, Cui K, Zhang L, Hou Y. Atorvastatin prevents contrast agent-induced renal injury in patients undergoing coronary angiography by inhibiting oxidative stress. Nan Fang Yi Ke Da Xue Хue Bao. 2012; 32: 1600-1602.

105. Gomes VO, Lasevitch R, Lima VC, et al. Hydration with sodium bicarbonate does not prevent contrast nephropathy: a 
multicenter clinical trial. Arq Bras Cardiol. 2012; 99: 1129-1134.

106. Gunebakmaz O, Kaya MG, Koc F, et al. Does nebivolol prevent contrast-induced nephropathy in humans? Clin Cardiol. 2012; 35: 250-254.

107. Hafiz AM, Jan MF, Mori N, et al. Prevention of contrast-induced acute kidney injury in patients with stable chronic renal disease undergoing elective percutaneous coronary and peripheral interventions: randomized comparison of two preventive strategies. Catheter Cardiovasc Interv. 2012; 79: 929-937.

108. Jaffery Z, Verma A, White CJ, et al. A randomized trial of intravenous n-acetylcysteine to prevent contrast induced nephropathy in acute coronary syndromes. Catheter Cardiovasc Interv. 2012; 79: 921-926.

109. Kitzler TM, Jaberi A, Sendlhofer G, et al. Efficacy of vitamin E and N-acetylcysteine in the prevention of contrast induced kidney injury in patients with chronic kidney disease: a double blind, randomized controlled trial. Wien Klin Wochenschrt. 2012; 124: 312-319.

110. Klima T, Christ A, Marana I, et al. Sodium chloride vs. sodium bicarbonate for the prevention of contrast mediuminduced nephropathy: a randomized controlled trial. Eur Heart J. 2012; 33: 2071-2079.

111. Koc F, Ozdemir K, Kaya MG, et al. Intravenous N-acetylcysteine plus high-dose hydration versus high-dose hydration and standard hydration for the prevention of contrast-induced nephropathy: CASIS--a multicenter prospective controlled trial. Int J Cardiol. 2012; 155: 418-423.

112. Li W, Fu X, Wang Y, et al. Beneficial effects of high-dose atorvastatin pretreatment on renal function in patients with acute ST-segment elevation myocardial infarction undergoing emergency percutaneous coronary intervention. Cardiology. 2012; 122: 195-202.

113. Okumura N, Hayashi M, Imai E, et al. Effects of carperitide on contrast-induced acute kidney injury with a minimum volume of contrast in chronic kidney disease patients. Nephron Extra. 2012; 2:303-310.

114. Quintavalle C, Fiore D, De Micco F, et al. Impact of a high loading dose of atorvastatin on contrast-induced acute kidney injury. Circulation. 2012; 126: 3008-3016.

115. Zhou L, Chen H. Prevention of contrast-induced nephropathy with ascorbic acid. Intern Med. 2012; 51: 531-535. 
116. Albabtain MA, Almasood A, Alshurafah H, Alamri H, Tamim H. Efficacy of ascorbic acid, N-acetylcysteine, or combination of both on top of saline hydration versus saline hydration alone on prevention of contrast-Induced nephropathy: a prospective randomized study. J Interv Cardiol. 2013; 26: 90-96.

117. Boucek P, Havrdova T, Oliyarnyk O, et al. Prevention of contrast-induced nephropathy in diabetic patients with impaired renal function: a randomized, double blind trial of sodium bicarbonate versus sodium chloride-based hydration. Diabetes Res Clin Pract. 2013; 101: 303-308.

118. Brueck M, Cengiz H, Hoeltgen R, et al. Usefulness of $\mathrm{N}$-acetylcysteine or ascorbic acid versus placebo to prevent contrast-induced acute kidney injury in patients undergoing elective cardiac catheterization: a single-center, prospective, randomized, double-blind, placebo-controlled trial. J Invasive Cardiol. 2013; 25:276-283.

119. Cicek M, Yildirir A, Okyay K, et al. Use of alpha-lipoic acid in prevention of contrast-induced nephropathy in diabetic patients. Ren Fail. 2013; 35: 748-753.

120. Dvorsak B, Kanic V, Ekart R, Bevc S, Hojs R. Ascorbic Acid for the prevention of contrast-induced nephropathy after coronary angiography in patients with chronic renal impairment: a randomized controlled trial. Ther Apher Dial. 2013; 17: 384-390.

121. Heguilen RM, Liste AA, Payaslian M, Ortemberg MG, Albarracin LM, Bernasconi AR. N-acethyl-cysteine reduces the occurrence of contrast-induced acute kidney injury in patients with renal dysfunction: a single-center randomized controlled trial. Clin Exp Nephrol. 2013; 17: 396-404.

122. Hui H, Li KL, Li ZB, et al. Effect of N-acetylcysteine for prevention contrast agent associated renal injury in elderly patients with coronary heart disease. Heart. 2013; 99: A255.

123. Jo SH, Kim SA, Kim HS, Han SJ, Park WJ, Choi YJ. Alpha-lipoic acid for the prevention of contrast-induced nephropathy in patients undergoing coronary angiography: the ALIVE study - a prospective randomized trial. Cardiology. 2013; 126: 159-166.

124. Koc F, Ozdemir K, Altunkas F, et al. Sodium bicarbonate versus isotonic saline for the prevention of contrast-induced nephropathy in patients with diabetes mellitus undergoing coronary angiography and/or intervention: a multicenter 
prospective randomized study. J Investig Med. 2013; 61: 872-877.

125. Maaz SH, Khandelwal PN, Baig MS, Doifode SM. A prospective, double blind, randomized, two-arm controlled clinical trial to determine the efficacy of atorvastatin $80 \mathrm{Mg}$ for the prevention of contrast induced nephropathy in mild chronic kidney disease patient undergoing angiography. Indian J Pharmacol. 2013; 45: S11.

126. Miao Y, Zhong Y, Yan H, Li W, Wang BY, Jin J. Alprostadil plays a protective role in contrast-induced nephropathy in the elderly. Int Urol Nephrol. 2013; 45: 1179-1185.

127. Poletti PA, Platon A, De Seigneux S, et al. N-acetylcysteine does not prevent contrast nephropathy in patients with renal impairment undergoing emergency CT: a randomized study. BMC Nephrol. 2013; 14:119.

128. Tasanarong A, Vohakiat A, Hutayanon P, Piyayotai D. New strategy of alpha- and -tocopherol to prevent contrast-induced acute kidney injury in chronic kidney disease patients undergoing elective coronary procedures. Nephrol Dial Transplant. 2013; 28: 337-344.

129. Traub SJ, Mitchell AM, Jones AE, et al. N-acetylcysteine plus intravenous fluids versus intravenous fluids alone to prevent contrast-induced nephropathy in emergency computed tomography. Ann Emerg Med. 2013; 62: 511-520.

130. Erturk M, Uslu N, Gorgulu S, et al. Does intravenous or oral high-dose N-acetylcysteine in addition to saline prevent contrast-induced nephropathy assessed by cystatin C? Coron Artery Dis. 2014; 25: 111-117.

131. Han Y, Zhu G, Han L, et al. Short-term rosuvastatin therapy for prevention of contrast-induced acute kidney injury in patients with diabetes and chronic kidney disease. J Am Coll Cardiol. 2014; 63:62-70.

132. Inda-Filho AJ, Caixeta A, Manggini M, Schor N. Do intravenous N-acetylcysteine and sodium bicarbonate prevent high osmolal contrast-induced acute kidney injury? A randomized controlled trial. PLOS ONE. 2014; 9: e107602.

133. Kama A, Yilmaz S, Yaka E, et al. Comparison of short-term infusion regimens of N-acetylcysteine plus intravenous fluids, sodium bicarbonate plus intravenous fluids, and intravenous fluids alone for prevention of contrast-induced nephropathy in the emergency department. Acad Emerg Med. 2014; 21:615-622.

134. Leoncini M, Toso A, Maioli M, Tropeano F, Villani S, Bellandi F. Early high-dose rosuvastatin for contrast-induced nephropathy prevention in acute coronary syndrome: Results from the PRATO-ACS Study (Protective Effect of Rosuvastatin 
and Antiplatelet Therapy On contrast-induced acute kidney injury and myocardial damage in patients with Acute Coronary Syndrome). J Am Coll Cardiol. 2014; 63: 71-79.

135. Li WH, Li DY, Qian WH, et al. Prevention of contrast-induced nephropathy with prostaglandin E1 in high-risk patients undergoing percutaneous coronary intervention. Int Urol Nephrol. 2014; 46:781-786.

136. Manari A, Magnavacchi P, Puggioni E, et al. Acute kidney injury after primary angioplasty: effect of different hydration treatments. J Cardiovasc Med. 2014; 15: 60-67.

137. Nieto-Rios JF, Salazar WA, Sanchez OM, et al. Prevention of contrast induced nephropathy with sodium bicarbonate (the PROMEC study). J Bras Nefrol. 2014; 36: 360-366.

138. Thayssen P, Lassen JF, Jensen SE, et al. Prevention of contrast-induced nephropathy with N-acetylcysteine or sodium bicarbonate in patients with ST-segment-myocardial infarction: a prospective, randomized, open-labeled trial. Circ Cardiovasc Interv. 2014; 7: 216-224.

139. Yang K, Liu W, Ren W, Lv S. Different interventions in preventing contrast-induced nephropathy after percutaneous coronary intervention. Int Urol Nephrol. 2014; 46:1801-1807.

140. Fahmy M, Eltahawy A. Role of rosuvastatin pretreatment in prevention of contrast induced nephropathy in patients undergoing coronary angiography. J Am Coll Cardiol.Vol 632014:S11.

141. Yeganehkhah MR, Iranirad L, Dorri F, et al. Comparison between three supportive treatments for prevention of contrastinduced nephropathy in high-risk patients undergoing coronary angiography. Saudi J Kidney Dis Transpl. 2014;25(6):12171223.

142. Abaci O, Arat Ozkan A, Kocas C, et al. Impact of Rosuvastatin on contrast-induced acute kidney injury in patients at high risk for nephropathy undergoing elective angiography. Am J Cardiol. Vol 1152015:867-871.

143. Arabmomeni M, Najafian J, Esfahani MA, Samadi M, Mirbagher L. Comparison between theophylline, Nacetylcysteine, and theophylline plus $\mathrm{N}$-acetylcysteine for the prevention of contrast-induced nephropathy. ARYA Atheroscler. 2015;11(1):43-49.

144. Bidram P, Roghani F, Sanei H, et al. Atorvastatin and prevention of contrast induced nephropathy following coronary 
angiography. J Res Med Sci. 2015;20(1):1-6.

145. Chong E, Poh KK, Lu Q, et al. Comparison of combination therapy of high-dose oral N-acetylcysteine and intravenous sodium bicarbonate hydration with individual therapies in the reduction of Contrast-induced Nephropathy during Cardiac Catheterisation and Percutaneous Coronary Intervention (CONTRAST): A multi-centre, randomised, controlled trial. Int J Cardiol. Vol 2012015:237-242.

146. Galal H, Nammas W, Samir A. Impact of high dose versus low dose atorvastatin on contrast induced nephropathy in diabetic patients with acute coronary syndrome undergoing early percutaneous coronary intervention. Egyptian Heart Journal. Vol 672015:329-336.

147. Jo SH, Hahn JY, Lee SY, et al. High-dose atorvastatin for preventing contrast-induced nephropathy in primary percutaneous coronary intervention. J Cardiovasc Med. 2015;16(3):213-219.

148. Shehata M, Hamza M. Impact of High Loading Dose of Atorvastatin in Diabetic Patients with Renal Dysfunction Undergoing Elective Percutaneous Coronary Intervention: A Randomized Controlled Trial. Cardiovasc Ther. 2015;33(2):3541.

149. Solomon R, Gordon P, Manoukian SV, et al. Randomized trial of bicarbonate or saline study for the prevention of contrast-induced nephropathy in patients with CKD. Clin JAm Soc Nephrol. 2015;10(9):1519-1524.

150. Rezaei Y, Khademvatani K, Rahimi B, Khoshfetrat M, Arjmand N, Seyyed-Mohammadzad MH. Short-Term High-Dose Vitamin E to Prevent Contrast Medium-Induced Acute Kidney Injury in Patients With Chronic Kidney Disease Undergoing Elective Coronary Angiography: A Randomized Placebo-Controlled Trial. J Am Heart Assoc. 2016;4(3):e002919. 
Table S2. Meta-analytic results of traditional pairwise meta-analysis

\begin{tabular}{|c|c|c|c|c|c|c|}
\hline Comparisons & $\mathbf{N}$ & $\mathbf{n}$ & $\tau^{2 \mathrm{a}}$ & $\mathrm{I}^{2} \%(95 \% \mathrm{Cl})$ & $\mathbf{Q}$ & $\begin{array}{l}\text { OR }(95 \% \mathrm{Cl})^{b} \text { from } \\
\text { traditional pairwise } \\
\text { meta-analysis }\end{array}$ \\
\hline $\begin{array}{l}\text { Vitamins and its analogues } \\
\text { vs. hydration }\end{array}$ & 13 & 2149 & 0 & $0(0,58)$ & 10.19 & $0.49(0.33,0.70)$ \\
\hline $\begin{array}{l}\text { Vitamins and its analogues } \\
\text { vs. NAC }\end{array}$ & 5 & 821 & 0 & $0(0,85)$ & 2.58 & $0.80(0.17,2.29)$ \\
\hline $\begin{array}{l}\text { Vitamins and its analogues } \\
\text { vs. bicarbonate sodium+NAC }\end{array}$ & 1 & 215 & NA & NA & NA & $6.70(1.46,30.68)$ \\
\hline $\begin{array}{l}\text { Natriuretic peptide vs. } \\
\text { hydration }\end{array}$ & 4 & 762 & 0.5 & $68(6,89)$ & 9.3 & $0.60(0.07,4.78)$ \\
\hline NAC vs. theophylline & 3 & 152 & 0.67 & $29(0,93)$ & 2.83 & $1.05(0.003,252)$ \\
\hline NAC vs. bicarbonate sodium & 11 & 1959 & 0.26 & $53(8,76)$ & 21.45 & $0.77(0.47,1.22)$ \\
\hline NAC vs. hydration & 70 & 12128 & 0.17 & $36(14,52)$ & 104.68 & $0.74(0.62,0.88)$ \\
\hline NAC vs. fenoldopam & 2 & 276 & 0 & $0^{c}$ & 0.55 & $0.32(0.006,147.8)$ \\
\hline $\begin{array}{l}\text { NAC vs. bicarbonate } \\
\text { sodium+NAC }\end{array}$ & 12 & 2792 & 0.63 & $65(35,81)$ & 31.53 & $1.19(0.61,2.14)$ \\
\hline $\begin{array}{l}\text { NAC vs. high-dose } \\
\text { statin+NAC }\end{array}$ & 6 & 1228 & 0.06 & $23(0,68)$ & 5.21 & $2.57(0.94,4.87)$ \\
\hline Prostaglandin vs. hydration & 5 & 943 & 0 & $0(0,79)$ & 1.87 & $0.35(0.17,0.65)$ \\
\hline $\begin{array}{l}\text { High-dose statin vs. low-dose } \\
\text { statin }\end{array}$ & 5 & 806 & 0 & $0(0,79)$ & 2.21 & $0.69(0.08,0.78)$ \\
\hline High-dose statin vs. hydration & 7 & 1437 & 0.1 & $21(0,64)$ & 7.59 & $0.38(0.18,0.71)$ \\
\hline Low-dose statin vs. hydration & 1 & 2998 & NA & NA & NA & $0.58(0.38,0.89)$ \\
\hline $\begin{array}{l}\text { Theophylline vs. bicarbonate } \\
\text { sodium }\end{array}$ & 1 & 280 & NA & NA & NA & $0.19(0.04,0.85)$ \\
\hline Theophylline vs. hydration & 9 & 739 & 0.39 & $32(0,69)$ & 11.73 & $0.55(0.18,1.21)$ \\
\hline $\begin{array}{l}\text { Bicarbonate sodium vs. } \\
\text { hydration }\end{array}$ & 28 & 5561 & 0.66 & $60(40,74)$ & 68.35 & $0.68(0.46,0.95)$ \\
\hline $\begin{array}{l}\text { Bicarbonate sodium vs. } \\
\text { bicarbonate sodium+NAC }\end{array}$ & 8 & 1598 & 0.13 & $25(0,66)$ & 9.28 & $1.21(0.70,2.04)$ \\
\hline Fenoldopam vs. hydration & 2 & 328 & 0.32 & $55^{c}$ & 2.26 & $0.76(0.009,516.7)$ \\
\hline $\begin{array}{l}\text { Bicarbonate sodium+ NAC } \\
\text { vs. hydration }\end{array}$ & 6 & 1194 & 0.06 & $1(0,75)$ & 5.07 & $1.21(0.70,2.04)$ \\
\hline $\begin{array}{l}\text { High-dose statin+NAC vs. } \\
\text { bicarbonate sodium+NAC }\end{array}$ & 1 & 410 & NA & NA & NA & $0.45(0.20,1.23)$ \\
\hline
\end{tabular}

Abbreviations: CI, confidence interval; N, number of trials; n, number of patients; NA, no available; NAC, $N$ acetylcysteine; OR, odds ratio, vs., versus.

${ }^{\text {a }} \tau^{2}$ represents between-study heterogeneity characterized by standard deviation.

${ }^{\mathrm{b}}$ the meta-regression based on empirical Bayes method was used to caculate ORs and 95CIs. ORs are lower than 1 favor the former treatment of every comparison. 


\section{Table S3. Evaluation of the model fit}

\begin{tabular}{|c|c|c|c|c|}
\hline Model assumption & Dbar & Pd & \# of data points & DIC \\
\hline Random consistency & 338 & 232 & 322 & $\mathbf{5 7 0}$ \\
\hline Random inconsistency & 343 & 236 & 322 & 579 \\
\hline Fixed consistency & 629 & 156 & 322 & 785 \\
\hline Fixed inconsistency & 502 & 168 & 322 & 630 \\
\hline
\end{tabular}

For a binomial likelihood each trial arm contributes 1 independent data point.

Dbar is considered as an absolute measure of fit, and is used to check formally whether a model's fit is satisfactory. This is the posterior mean of the deviance under the current model minus the deviance for the saturated mode. We can then compare the value of Dbar to the number of independent data points to check if the model fit can be improved.

Leverage $\left(\boldsymbol{P}_{\boldsymbol{D}}\right)$ is considered an appropriate measure of the complexity of a model that reasonably describes the data. $P_{D}$ also is termed the effective number of parameters, and is calculated as the posterior mean of the residual deviance minus the deviance at the posterior mean of the fitted values.

Deviance Information Criterion (DIC) is the sum of the posterior mean of the residual deviance and the $P_{D}$, and provides a measure of model fit that penalises model complexity - lower values of the DIC suggest a more parsimonious model. The DIC is particularly useful for comparing different parameter models for the same likelihood and data, for example fixed and random effects models or fixed effect models with and without covariates. As shown in above table, the random consistency model is clearly more parsimonious than the other three models. 
Table S4. Results of sensitivity analyses

\begin{tabular}{|c|c|c|c|c|c|c|c|}
\hline Treatment strategies & $\begin{array}{l}\text { Standard } \\
\text { analysis }\end{array}$ & $\begin{array}{c}\text { Excluding } 13 \\
\text { trials with } \\
\text { sample size less } \\
\text { than } 50\end{array}$ & $\begin{array}{c}\text { Excluding } 18 \text { trials } \\
\text { with high-osmolar } \\
\text { and unspecified CM } \\
\text { type }\end{array}$ & $\begin{array}{c}\text { Excluding } 14 \\
\text { trials with oral } \\
\text { hydration and } \\
\text { unspecified } \\
\text { hydration agent }\end{array}$ & $\begin{array}{c}\text { Excluding } 9 \\
\text { trials evaluated } \\
\text { only patients } \\
\text { with normal } \\
\text { kidney function }\end{array}$ & $\begin{array}{l}\text { Excluding } \\
\text { data for non- } \\
\text { DM patients }\end{array}$ & $\begin{array}{l}\text { Excluding } 24 \\
\text { trials published } \\
\text { before } 2004\end{array}$ \\
\hline High-dose statin+NAC & $0.31(0.14,0.60)$ & $0.32(0.15,0.58)$ & $0.32(0.14,0.64)$ & $0.38(0.15,0.83)$ & $0.32(0.14,0.66)$ & $0.24(0.07,1.27)$ & $0.33(0.16,0.60)$ \\
\hline High-dose statin & $0.37(0.19,0.64)$ & $0.38(0.21,0.64)$ & $0.39(0.21,0.68)$ & $0.35(0.17,0.62)$ & $0.42(0.20,0.72)$ & $0.47(0.11,1.33)$ & $0.38(0.21,0.62)$ \\
\hline Prostaglandin & $0.37(0.17,0.72)$ & $0.37(0.17,0.68)$ & $0.43(0.16,0.86)$ & $0.40(0.17,0.74)$ & $0.45(0.21,0.87)$ & - & $0.42(0.16,0.88)$ \\
\hline Theophylline & $0.48(0.26,0.82)$ & $0.46(0.24,0.82)$ & $0.46(0.26,0.75)$ & $0.55(0.29,0.93)$ & $0.48(0.23,0.85)$ & $0.77(0.01,4.69)$ & $0.60(0.27,1.14)$ \\
\hline Bicarbonate sodium+NAC & $0.62(0.40,0.88)$ & $0.57(0.38,0.80)$ & $0.47(0.30,0.69)$ & $0.54(0.36,0.77)$ & $0.55(0.37,0.79)$ & $1.14(0.46,2.42)$ & $0.56(0.37,0.82)$ \\
\hline Vitamins and its analogues & $0.64(0.41,0.95)$ & $0.64(0.42,0.96)$ & $0.58(0.38,0.87)$ & $0.63(0.41,0.95)$ & $0.62(0.38,0.93)$ & $0.87(0.43,1.57)$ & $0.75(0.48,1.13)$ \\
\hline NAC & $0.67(0.54,0.81)$ & $0.71(0.58,0.87)$ & $0.64(0.52,0.77)$ & $0.67(0.54,0.81)$ & $0.66(0.54,0.81)$ & $0.81(0.54,1.14)$ & $0.73(0.59,0.88)$ \\
\hline Natriuretic peptide & $0.69(0.31,1.37)$ & $0.71(0.31,1.40)$ & $0.70(0.32,1.34)$ & $0.71(0.30,1.31)$ & $0.71(0.31,1.35)$ & $1.91(0.45,5.71)$ & $0.62(0.27,1.30)$ \\
\hline Fenoldopam & $0.70(0.32,1.36)$ & $1.69(0.68,3.44)$ & $1.22(0.54,2.48)$ & $1.26(0.57,2.44)$ & $1.26(0.55,2.52)$ & $0.95(0.41,1.85)$ & $2.53(0.69,7.05)$ \\
\hline Bicarbonate sodium & $0.78(0.59,1.01)$ & $0.79(0.60,1.00)$ & $0.66(0.49,0.84)$ & $0.77(0.58,0.99)$ & $0.78(0.59,1.01)$ & $1.31(0.67,2.38)$ & $0.78(0.59,0.99)$ \\
\hline Low-dose statin & $0.98(0.41,2.07)$ & $0.96(0.44,1.90)$ & $0.89(0.39,1.72)$ & $0.74(0.30,1.52)$ & $1.04(0.41,2.11)$ & $0.65(0.20,1.52)$ & $0.95(0.42,1.85)$ \\
\hline Hydration & Reference & Reference & Reference & Reference & Reference & Reference & Reference \\
\hline Heterogeneity & $0.33(0.21,0.54)$ & $0.35(0.19,0.56)$ & $0.30(0.16,0.49)$ & $0.34(0.19,0.55)$ & $0.35(0.19,0.54)$ & $0.15(0.00,0.71)$ & $0.29(0.15,0.50)$ \\
\hline DIC & 570 & 521 & 500 & 522 & 541 & 142 & 480 \\
\hline Number of trials & 150 & 137 & 132 & 125 & 141 & 38 & 126 \\
\hline Heterogeneity change & & rise $6 \%$ & drop 9\% & rise $3 \%$ & rise $6 \%$ & drop 55\% & drop $12 \%$ \\
\hline
\end{tabular}

Data are odds ratio (95\% CrI). All odds ratios use hydration as referenced agent. Heterogeneity was assessed using the posterior median between trial variance, $\tau^{2}$. Significant results are in bold. CM, contrast media; CrI, credible interval; DM, Diabetes mellitus; SUCRA, surface under the cumulative ranking curve measure; NAC, $N$-acetylcysteine; 
Table S5. Results of meta-regression and subgroup analyses

\begin{tabular}{|c|c|c|c|c|c|c|c|c|}
\hline $\begin{array}{l}\text { Treatment } \\
\text { strategies }\end{array}$ & $\begin{array}{l}\text { Standard } \\
\text { analysis }\end{array}$ & $\begin{array}{c}\text { Mean CM } \\
\text { dose }\end{array}$ & $\begin{array}{l}\text { Baseline Scr } \\
\text { concentration }\end{array}$ & $\begin{array}{c}\text { Mean age } \\
\text { years }\end{array}$ & $\begin{array}{l}\text { CM type (iso-, } \\
\text { low- or high- } \\
\text { osmolar) }\end{array}$ & $\begin{array}{c}\text { Isotonic } \\
(0.9 \%) \text { or } \\
\text { hypotonic } \\
(0.45 \%) \text { saline } \\
\text { hydration } \\
\end{array}$ & $\begin{array}{c}\text { Different CI- } \\
\text { AKI } \\
\text { definitions(48h } \\
\text {,72h or 120h) }\end{array}$ & $\begin{array}{l}\text { Cardiovascular diagnostic/ } \\
\text { interventional procedures } \\
\text { or enhanced CT or not } \\
\text { specified radiologic } \\
\text { procedure with CM }\end{array}$ \\
\hline $\begin{array}{l}\text { High-dose } \\
\text { statin+NAC }\end{array}$ & $0.31(0.14,0.60)$ & $0.35(0.14,0.69)$ & $0.29(0.13,0.55)$ & $0.35(0.15,0.72)$ & $0.33(0.14,0.65)$ & $0.40(0.15,0.86)$ & $0.33(0.14,0.66)$ & $0.29(0.13,0.54)$ \\
\hline $\begin{array}{l}\text { High-dose } \\
\text { statin }\end{array}$ & $0.37(0.19,0.64)$ & $0.36(0.15 .0 .76)$ & $0.34(0.16,0.64)$ & $0.35(0.17,0.68)$ & $0.44(0.21,0.80)$ & $0.39(0.17,0.75)$ & $0.36(0.17,0.68)$ & $0.33(0.17,0.58)$ \\
\hline Prostaglandin & $0.37(0.17,0.72)$ & $0.40(0.14,0.89)$ & $0.38(0.17,0.74)$ & $0.37(0.17,0.76)$ & $0.37(0.14,0.70)$ & $0.43(0.19,0.82)$ & $0.35(0.15,0.68)$ & $0.47(0.20,0.92)$ \\
\hline Theophylline & $0.48(0.26,0.82)$ & $0.46(0.24,0.84)$ & $0.47(0.25,0.79)$ & $0.52(0.28,0.90)$ & $0.48(0.25,0.84)$ & $0.59(0.31,0.99)$ & $0.45(0.24,0.77)$ & $0.46(0.25,0.77)$ \\
\hline $\begin{array}{l}\text { Bicarbonate } \\
\text { sodium+NAC }\end{array}$ & $0.62(0.40,0.88)$ & $0.54(0.35,0.80)$ & $0.51(0.33,0.73)$ & $0.55(0.37,0.79)$ & $0.52(0.34,0.75)$ & $0.57(0.36,0.84)$ & $0.45(0.29,0.69)$ & $0.52(0.34,0.75)$ \\
\hline $\begin{array}{l}\text { Vitamins and } \\
\text { its analogues }\end{array}$ & $0.64(0.41,0.95)$ & $0.62(0.39,0.97)$ & $0.63(0.39,0.96)$ & $0.57(0.32,0.90)$ & $0.88(0.41,1.67)$ & $0.66(0.43,0.97)$ & $0.70(0.45,1.03)$ & $0.72(0.48,1.02)$ \\
\hline NAC & $0.67(0.54,0.81)$ & $0.66(0.52,0.81)$ & $0.66(0.52,0.82)$ & $0.67(0.54,0.83)$ & $0.65(0.53,0.79)$ & $0.70(0.56,0.89)$ & $0.67(0.53,0.82)$ & $0.64(0.51,0.77)$ \\
\hline $\begin{array}{l}\text { Natriuretic } \\
\text { peptide }\end{array}$ & $0.69(0.31,1.37)$ & $0.71(0.30,1.40)$ & $0.71(0.31,1.35)$ & $0.71(0.33,1.42)$ & $0.70(0.30,1.45)$ & $0.75(0.33,1.51)$ & $0.91(0.33,1.88)$ & $0.66(0.30,1.27)$ \\
\hline Fenoldopam & $0.70(0.32,1.36)$ & $1.25(0.47,2.47)$ & $1.25(0.54,2.47)$ & $1.27(0.56,2.49)$ & $1.25(0.53,2.39)$ & $1.43(0.57,2.94)$ & $1.21(0.52,2.35)$ & $1.18(0.52,2.35)$ \\
\hline $\begin{array}{l}\text { Bicarbonate } \\
\text { sodium }\end{array}$ & $0.78(0.59,1.01)$ & $0.78(0.59,1.02)$ & $0.77(0.58,0.99)$ & $0.78(0.60,1.01)$ & $0.67(0.49,0.88)$ & $0.79(0.59,1.04)$ & $0.75(0.55,1.04)$ & $0.74(0.55,0.96)$ \\
\hline $\begin{array}{l}\text { Low-dose } \\
\text { statin }\end{array}$ & $0.98(0.41,2.07)$ & $0.92(0.33,2.10)$ & $0.91(0.37,1.92)$ & $0.94(0.32,2.18)$ & $1.00(0.38,2.50)$ & $0.79(0.31,1.65)$ & $0.91(0.36,1.90)$ & $0.83(0.34,1.68)$ \\
\hline Hydration & Reference & Reference & Reference & Reference & Reference & Reference & Reference & Reference \\
\hline Heterogeneity & $0.33(0.21,0.54)$ & $0.38(0.21,0.60)$ & $0.34(0.19,0.56)$ & $0.33(0.19,0.54)$ & $0.30(0.18,0.52)$ & $0.33(0.20,0.55)$ & $0.33(0.19,0.58)$ & $0.33(0.20,0.55)$ \\
\hline B coefficient & & $\begin{array}{c}0.001(- \\
0.008,0.007) \\
\end{array}$ & $\begin{array}{c}-0.27(- \\
2.04,1.48) \\
\end{array}$ & $\begin{array}{c}-0.05(- \\
0.17,0.07) \\
\end{array}$ & $\begin{array}{c}0.45(- \\
0.21,1.18) \\
\end{array}$ & $\begin{array}{c}-0.21(- \\
0.58,0.19) \\
\end{array}$ & $\begin{array}{c}0.09(- \\
0.19,0.35) \\
\end{array}$ & $0.09(-0.10,0.28)$ \\
\hline DIC & 570 & 502 & 534 & 530 & 519 & 517 & 501 & 571 \\
\hline $\begin{array}{l}\text { Heterogeneity } \\
\text { change }\end{array}$ & & rise $15 \%$ & rise 3\% & $0 \%$ & drop 9\% & $0 \%$ & $0 \%$ & $0 \%$ \\
\hline
\end{tabular}

Data are odds ratio (95\% CrI) after adjusting covariates: a. continuous variables include "Mean CM dose", "Baseline scr concentration", and "Mean age years";

b. categorical variables include "CM type (iso-, low- or high-osmolar)", "Isotonic (0.9\%) or hypotonic (0.45\%) saline hydration", "Different CI-AKI definitions (48h,72h or 120h)", "Cardiovascular diagnostic/interventional procedures or enhanced CT or not specified radiologic procedure with CM". All odds ratios use 
hydration as referenced agent. Heterogeneity was assessed using the posterior median between trial variance, $\tau^{2}$. Significant results are in bold. CM, contrast media CrI, credible interval; CT, computed tomography; Scr, Serum creatinine; NAC, $N$-acetylcysteine. 


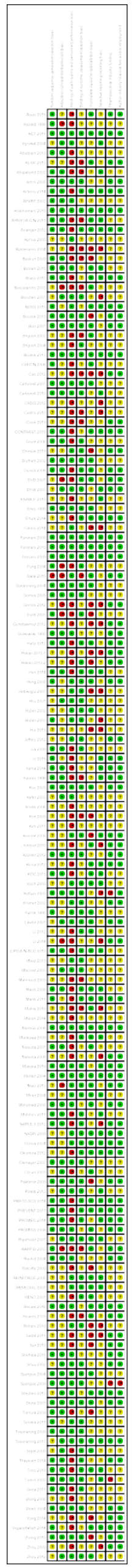


Random sequence generation(selection biaste

Allocation concealment(selection bias)

Blinding of participants and personnel(performance bias)

Blinding of outcome assessment(detection bias)

Incomplete outcome data(attrition bias)

Selective reporting(reporting bias)

Pharmaceutical industry funding

Author-industry financial ties and/or employment
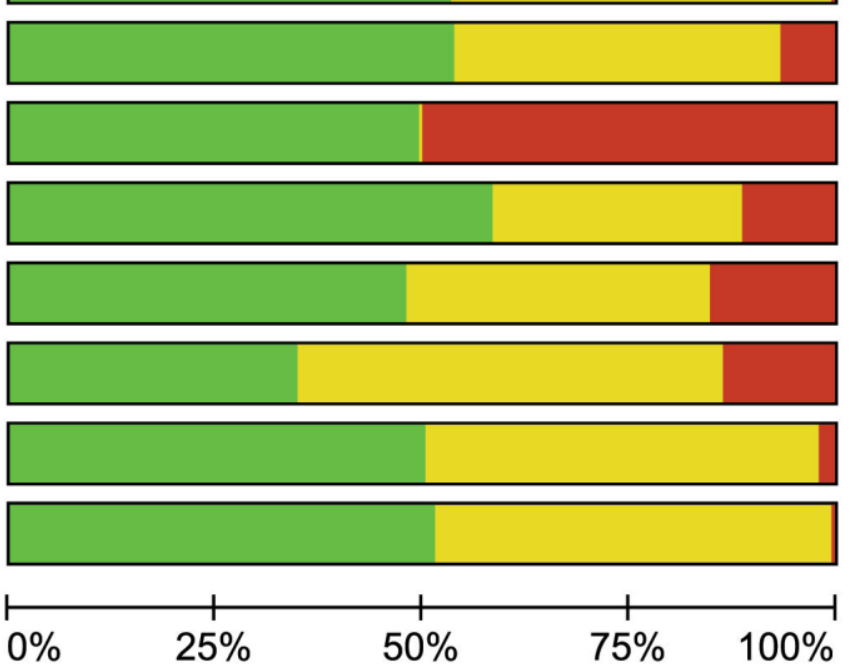


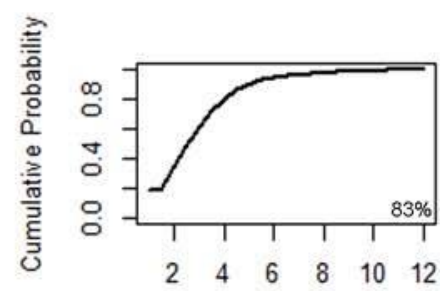

Rank of High-dose statin

Rank of High-dose statin+NAC

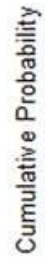

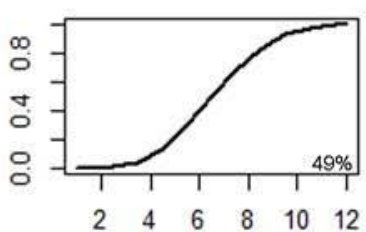

Rank of Bicarbonate sodium +NAC

흫

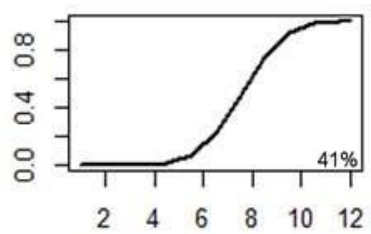

Rank of N-acetylcysteine
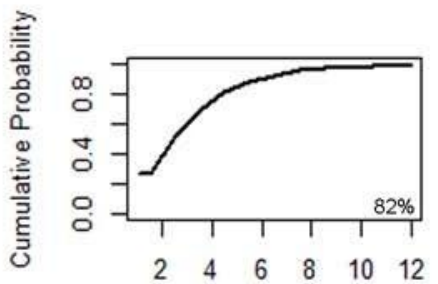

Rank of Prostaglandin

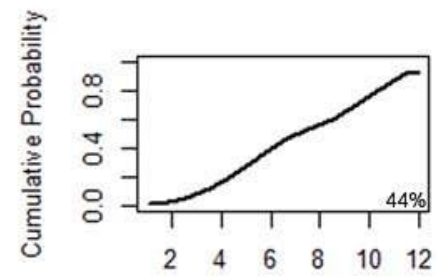

Rank of Natriuretic peptide

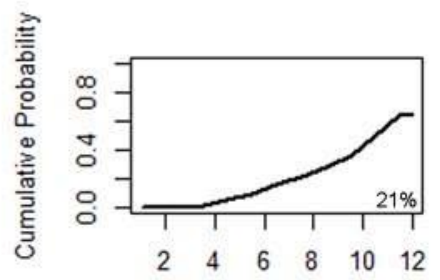

Rank of Low-dose statin

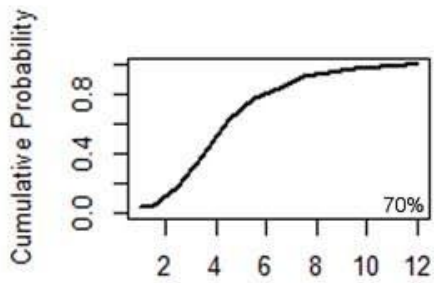

Rank of Theophylline

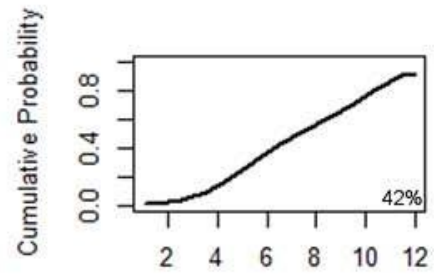

Rank of Fenoldopam

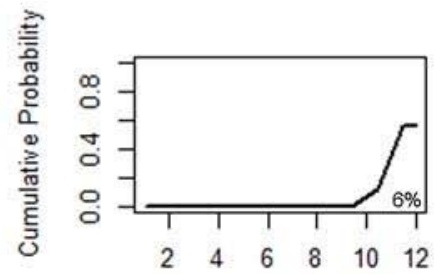

Rank of Hydration

\section{Non-cumulative ranking curves}

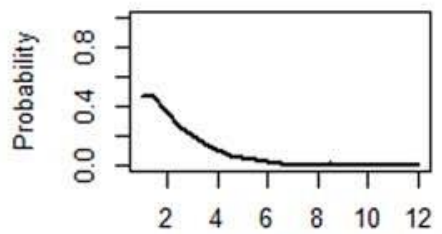

Rank of High-dose statin+NAC

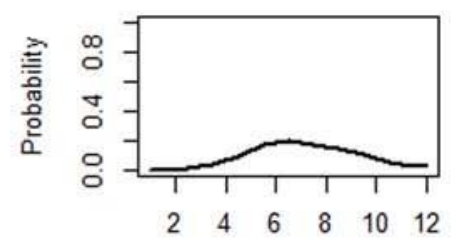

Rank of Bicarbonate sodium +NAC

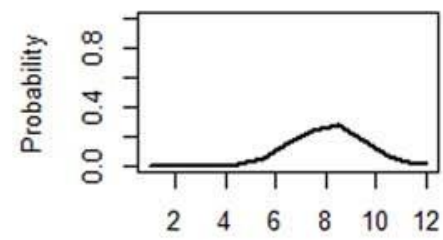

Rank of $\mathrm{N}$-acetylcysteine

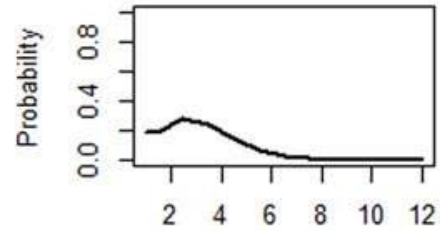

Rank of High-dose statin

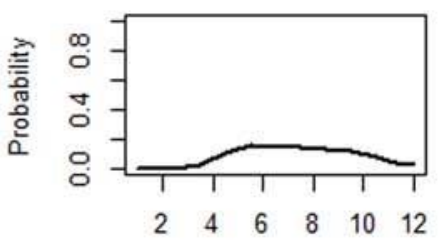

Rank of Vitamin and its analogues

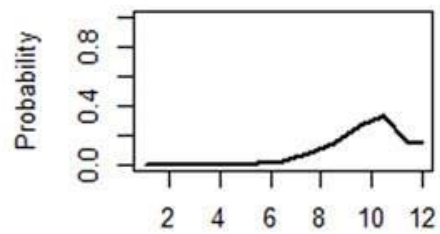

Rank of Bicarbonate sodium

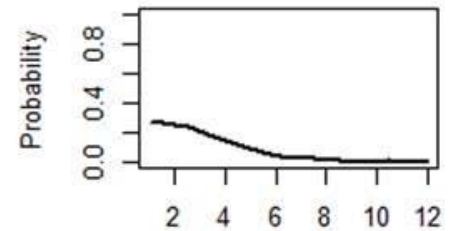

Rank of Prostaglandin

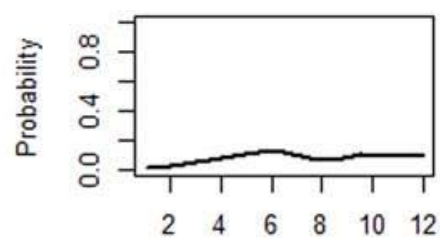

Rank of Natriuretic peptide

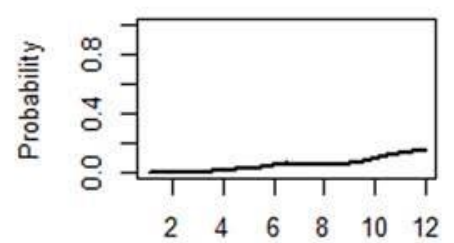

Rank of Low-dose statin

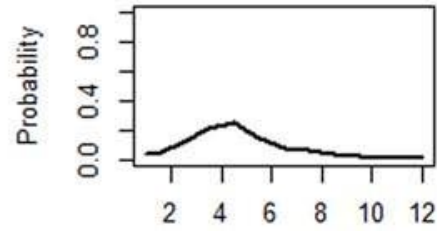

Rank of Theophylline

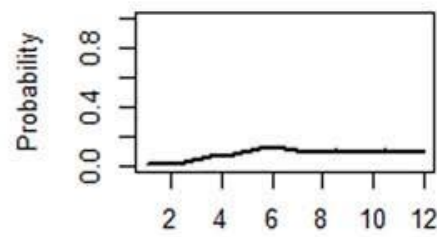

Rank of Fenoldopam

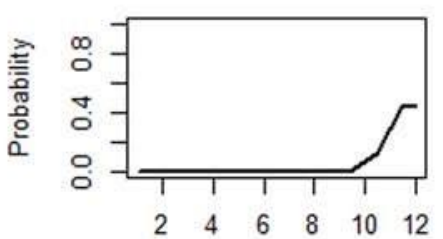

Rank of Hydration 
3.7.8.

11.3.8.

3.8.9.

7.8.9.

11.8.9.

1.11.3.

1.3.9.

1.11.9.

10.3.9.

11.12.3.

3.7.9.

11.3.9.

5.6.9.

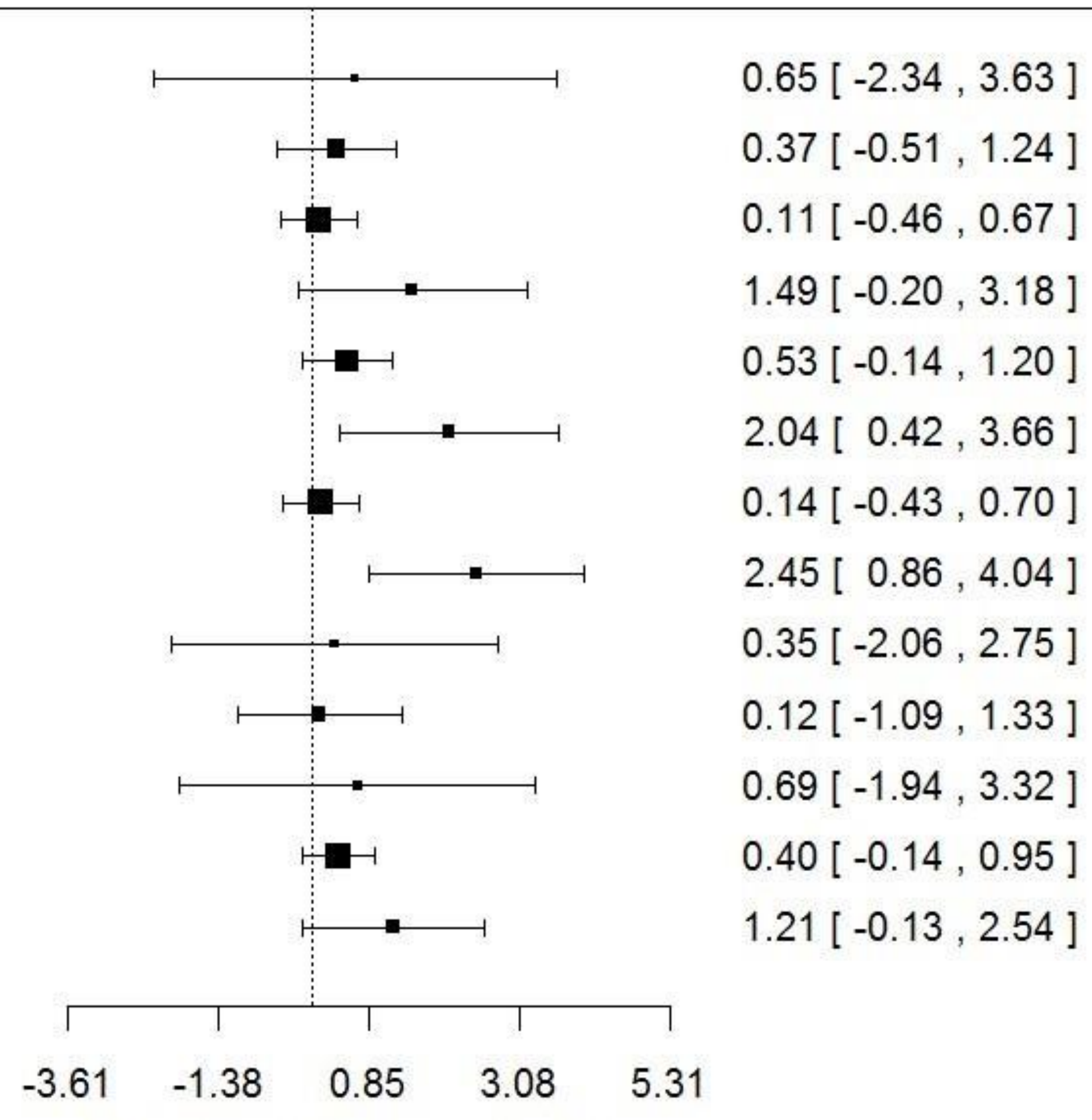

Inconsistency for triangular loops 
Item S1. Study protocol:

PROTOCOL

First drafted in October 2014

Modified in January 2015

Comparative Effectiveness of 12 Treatment Strategies in Preventing

Contrast-Induced Acute Kidney Injury: A Systematic Review and Bayesian Network Meta-analysis

Xiaole Su, Xinfang Xie, Lijun Liu, Jicheng Lv, Fujian Song, Perkovic Vlado and Hong Zhang 


\section{Objectives:}

Integrate the available evidence to compare various types of pharmacological strategies when used in patients undergoing diagnostic and/or interventional procedures with contrast media(CM) and create hierarchies of the comparative efficacy of active drug treatments on preventing contrast-induced acute kidney injury (CI-AKI).

\section{Background}

Acute injure in renal function induced by CM is generally mild and transient but can result in lasting renal dysfunction and the need for renal replacement therapy. CI-AKI is a leading cause of new onset kidney injury in hospitalized patients $(1,2)$. It is associated with significantly increased in-hospital morbidity and mortality, acceleration of chronic kidney disease, and increased costs of medical care (3). There have been a large number of pharmacological strategies to prevent CI-AKI so far, such as $\mathrm{N}$ acetylcysteine (NAC), theophylline, fenoldopam, dopamine, iloprost, statins, bicarbonate sodium, ascorbic acid (vitamin C), vitamin E and. The question of which treatment strategies should be preferred for the prevention of CI-AKI is controversial, and traditional meta-analyses are hindered by heterogeneity across trials and the lack of trials direct comparing different treatment agents. We will undertake a network metaanalysis, which accounts for both direct and indirect comparisons to assess the efficacy of treatments on preventing CI-AKI.

\section{Research Plan:}

\section{A) Methods of the Review}

We will conduct a Bayesian-framework, multiple-treatments meta-analysis (which uses 
both direct and indirect comparisons) of randomized controlled trials (RCTs) (4).

\section{B) Data Sources:}

Relevant RCTs will be identified by computerized searches from the following data sources without language restriction:

1) MEDLINE OVID SP (from 1947 through October 2014);

2) EMBASE (from 1966 through October 2014);

3) The Cochrane Central Register of Controlled Trials (no date restriction);

4) Reference lists in nephrology textbooks, review articles, and relevant trials were also searched.

\section{C) Study Selection:}

\section{Types of Studies:}

- Inclusion criteria:

We will include RCTs compared two or more of treatment groups received: NAC, theophylline (aminophylline), fenoldopam, iloprost, alprostadil, prostaglandin E1, statins, statins plus NAC, bicarbonate sodium, bicarbonate sodium plus NAC, ascorbic acid, vitamin E, tocopherol, alpha-lipoic acid, atrial natriuretic peptide, B-type natriuretic peptide, carperitide and hydration or placebo plus hydration. All above active drug treatments were based on hydration. All participants underwent diagnostic and/or interventional procedures with CM.

- Exclusion criteria:

Trials contained only one or none of the above strategies.

\section{Types of Participants:}


- Inclusion criteria:

Adult patients (age $\geq 18$ years) underwent diagnostic and/or interventional procedures with CM.

- Exclusion criteria: None

\section{Type of Intervention:}

- Treatment groups:

$\mathrm{N}$-acetylcysteine, theophylline (aminophylline), fenoldopam, iloprost, alprostadil, prostaglandin E1, statins, statins plus NAC, bicarbonate sodium, bicarbonate sodium plus NAC, ascorbic acid, vitamin E or its analogues (tocopherol), alpha-lipoic acid, atrial natriuretic peptide, B-type natriuretic peptide, carperitide and hydration or placebo plus hydration;

- Compared two or more of the above mentioned treatment agents;

- All above active drug treatments were based on hydration.

\section{Type of Outcome Measures:}

- Primary Outcome

The occurrence of CI-AKI, defined as an absolute increase in baseline serum creatinine greater than $44.2 \mu \mathrm{mol} / \mathrm{L}(0.5 \mathrm{mg} / \mathrm{dL})$ or a relative increase greater than $25 \%$ within typically 48-72 h after contrast injection. If 48-72 h data were not available, we used data within 5 days (the data point closest to 48-72 h was given preference).

- Secondary Outcome

None

\section{D) Assess Study Ouality:}


We will use the Cochrane risk of bias method to appraise study quality on the seven domains (low, unclear, or high bias for sequence generation; allocation concealment; blinding of participants, personnel and outcome assessors; incomplete outcome data; selective outcome reporting; and other sources of bias) (5).

\section{E) Statistical analysis}

We will use Stata 12.0 to perform the traditional pairwise meta-analysis. Bayesian network meta-analysis will be done with WinBUGS version 1.4.3 and the R2WinBUGS package of R software 3.1.1. Clinical outcome analyses were compared by odds ratios (ORs) and 95\% credible intervals (CrIs) using a Bayesian hierarchical random-effects model. Model fit will be assessed by comparing deviance information criterion (DIC). We will use the surface under the cumulative ranking (SUCRA) probabilities to rank the treatments (6). We will estimate the absolute difference between direct and indirect estimates in each closed loop. A significant (95\% CrI that excludes 0) disagreement between direct and indirect evidence will indicate Inconsistent loops $(7,8)$.

We will do multiple-treatments meta-regression with the following covariates: mean age, mean CM dose, and baseline serum creatinine concentration (7). Subgroup analyses will be performed by comparing with trials using with different CI-AKI definitions, and comparing with trials of cardiovascular diagnostic/interventional procedures and CT examination (7). Sensitivity analyses will be conducted by only including of trials of DM patients and by excluding of trials with small sample size and trails of high-osmolar CM used.

\section{F) The Search Strategy:}




\section{1) MEDLINE OVID SP}

1. exp Acute Kidney Injury/

2. exp renal failure/

3. (kidney disease* or renal disease* or renal failure or kidney failure or acute kidney or acute renal or nephrotoxic or nephropathy).mp.

4. (impair or injury or damage or reduce).mp. and (renal or kidney).mp.

5. 1 or 2 or 3 or 4

6. (contrast-induced or contrast-associated).mp.

7. (contrast or radiocontrast or iopamidol or iodine or ioxaglic acid or iodine compounds) .mp.

8. (iohexol or urography or tomography or X ray computed or diatrizoate).mp.

9. 6 or 7 or 8

10. randomized controlled trial .pt.

11. controlled clinical trial.pt.

12. randomized.ab.

13. placebo. ab.

14. clinical trials as topic.sh

15. randomly.ab.

16. trial.ti.

17. 10 or 11 or 12 or 13 or 14 or 15 or 16

18. animals.sh. not (humans.sh. and animals.sh.)

19. 17 not 18 
20. 5 and 9 and 19

\section{2) EMBASE}

\#1 ' Acute Kidney Injury'/exp

\#2 ' renal failure'/exp

\#3 'kidney disease\$' or 'renal disease\$' or 'renal failure' or 'kidney failure' or 'acute kidney' or 'acute renal' or nephrotoxic or nephropathy

\#4 (impair or injury or damage or reduce) and (renal or kidney)

\#5 \#1 OR \#2 OR \#3 OR \#4

\#6 'contrast-induced' or 'contrast-associated'

\#7 contrast or radiocontrast or iopamidol or iodine or 'ioxaglic acid' or 'iodine compound $\$$

\#8 iohexol or urography or tomography or ' $\mathrm{X}$ ray computed' or diatrizoate

\#9 \#6 OR \#7 OR \#8

\#10 random\$ OR blind\$ OR placebo OR 'meta analysis'

\#11 \#5 AND \#9 AND \#10

\section{3) CENTRAL}

\#1 MeSH descriptor: [Acute Kidney Injury] explode all trees

\#2 MeSH descriptor: [renal failure] explode all trees

\#3 kidney disease* or renal disease* or renal failure or kidney failure or acute kidney or acute renal or nephrotoxic or nephropathy 
\#4 (impair or injury or damage or reduce) and (renal or kidney)

\#5 \#1 or \#2 or \#3 or \#4

\#6 contrast-induced or contrast-associated

\#7 contrast or radiocontrast or iopamidol or iodine or ioxaglic acid or iodine

compound*

\#8 iohexol or urography or tomography or $\mathrm{X}$ ray computed or diatrizoate

\#9 \#6 or \#7 or \#8

\#10 \#5 and \#9

4) Reference lists of nephrology textbooks, review articles, and relevant trials were also searched.

\section{Reference}

1. Hou SH, Bushinsky DA, Wish JB, Cohen JJ, Harrington JT. Hospital-acquired renal insufficiency: a prospective study. Am J Med. 1983; 74(2): 243-8.

2. Levy EM, Viscoli CM, Horwitz RI. The effect of acute renal failure on mortality. A cohort analysis. JAMA. 1996; 275(19): 1489-94.

3. Rihal CS, Textor SC, Grill DE, et al. Incidence and prognostic importance of acute renal failure after percutaneous coronary intervention. Circulation. 2002; 105(19): 2259-64.

4. Liberati A, Altman DG, Tetzlaff J, et al. The PRISMA statement for reporting systematic reviews and meta-analyses of studies that evaluate healthcare interventions: explanation and elaboration. BMJ. 2009; 339: b2700.

5. Higgins JP, Altman DG, Gotzsche PC, et al. The Cochrane Collaboration's tool for assessing risk of bias in randomised trials. BMJ. 2011; 343: d5928. 
6. Salanti G, Ades AE, Ioannidis JP. Graphical methods and numerical summaries for presenting results from multiple-treatment meta-analysis: an overview and tutorial. J Clin Epidemiol. 2011; 64(2): 163-71.

7. Dias S, Sutton AJ, Welton NJ, Ades AE. NICE DSU technical support document 3: heterogeneity: subgroups, meta-regression, bias and bias-adjustment report by the decision support unit. September 2011 (last updated April 2012). http://www.nicedsu.org.uk/TSD3\%20 Heterogeneity.final\%20report.08.05.12.pdf (accessed Feb 12, 2015).

8. Dias S, Welton NJ, Sutton AJ, Caldwell DM, Lu G, Ades AE. NICE DSU technical support document 4: inconsistency in networks of evidence based on randomised controlled trials report by the decision support unit. May 2011(last updated April 2014). http://www.nicedsu.org.uk/ TSD4\%20Inconsistency.final.15April2014.pdf (accessed Feb 12, 2015). 


\section{Item S2. Assessment domains of risk of bias}

We assessed risk of bias for sequence generation, allocation concealment, blinding, selective reporting, incomplete outcome data and other sources of bias, and determined overall risk of bias based on predefined rules, utilizing the Cochrane Collaboration risk of bias tool. ${ }^{1}$

\section{Sequence generation (Selection bias)}

- Low risk of bias, if randomization was generated by a computer, or a table of random numbers.

- High risk of bias, if method of randomization was inadequate (i.e. "quasirandomized").

- Unclear risk of bias, if method of randomization was not described.

\section{Allocation concealment (Selection bias)}

- Low risk of bias, if the method of allocation involved a central independent unit or consecutively numbered sealed envelopes.

- High risk of bias, if allocation sequence was known to the investigators or conducted with an inadequate method.

- Unclear risk of bias, if the method of allocation concealment was not described.

\section{Blinding of participants and personnel (Performance bias)}

- Low risk of bias, if the study was of a double-blind design.

- High risk of bias, if the study was open-label.

- Unclear risk of bias, if there was insufficient information to determine whether the study was double-blind or open-label. 


\section{Blinding of outcome assessment (Detection bias)}

- Low risk of bias, if the outcome assessment was blind.

- High risk of bias, if the outcome assessment was open.

- Unclear risk of bias, if there was insufficient information to determine whether the outcome assessment was blind or open.

\section{Selective outcome reporting (Detection bias)}

- Low risk of bias, if the specific outcome was reported adequately for all treatment arms.

- High risk of bias, if the specific outcome was reported with inadequate detail for the data to be included in a meta-analysis or if it was reported only for a subset of the randomized population.

- Unclear risk of bias, if there was insufficient information to assess whether the risk of bias of selective outcome reporting was present.

\section{Incomplete outcome data (Attrition bias)}

- Low risk of bias, if

1. attrition rate was balanced between treatment arms and relatively low (below 20\%), and

2. reasons for discontinuation were described, and

3. an intention-to-treat analysis was performed, and

4. an appropriate method of imputation of missing outcome data was applied.

- High risk of bias, if

1. withdrawal rates were unbalanced between treatment arms or more than $20 \%$, or 
2. reasons for drop-outs were not clearly described, or

3. an inappropriate analysis was performed (i.e. per protocol analysis), or

4. an inappropriate imputation method (i.e. last observation carried forward method) was used to handle missing data.

- Unclear risk of bias, if it is not clear whether there were any drop-outs, or reasons for these withdrawals are not clear, or no method of imputation of missing data is mentioned.

\section{Pharmaceutical industry funding (Sponsor bias) ${ }^{2}$}

- Low risk of bias, if the trial was not funded by a drug manufacturer.

- High risk of bias, if the trial was funded by a drug manufacturer.

- Unclear risk of bias, if the source of funding was unclear.

\section{Author-industry financial ties and/or employment (Other bias) ${ }^{2}$}

- Low risk of bias, if any authors did not disclose financial ties and/or employment by the pharmaceutical industry.

- High risk of bias, if any authors disclose financial ties and/or employment by the pharmaceutical industry.

- Unclear risk of bias, if author-industry financial ties or affiliation were not reported.

\section{Reference}

1. Higgins JP, Altman DG, Gotzsche PC, Juni P, Moher D, Oxman AD, Savovic J, Schulz KF, Weeks L, Sterne JA, Cochrane Bias Methods G, Cochrane Statistical Methods G. The Cochrane Collaboration's tool for assessing risk of bias in randomised 
trials. BMJ. 2011;343:d5928.

2. Roseman M, Milette K, Bero LA, et al. Reporting of conflicts of interest in metaanalyses of trials of pharmacological treatments. JAMA. 2011;305(10):1008-1017. 


\section{Item S3 Statistical method}

(A short summary, see details http://www.mtm.uoi.gr. and http://www.nicedsu.org.uk.)

\section{Bayesian approach and Credible intervals (CrI)}

The Bayesian approach utilizes both sample data and prior knowledge in estimating validity and weights each in proportion to its information value. The sample information is combined with the prior distribution to produce a posterior distribution, the mean of which is then taken as the estimate of the parameter of interest, in this case, test validity. Confidence intervals — called credible intervals by Bayesians — can be placed around this mean ${ }^{1}$.

Confidence intervals (CI) usually is used in conventional non-Bayesian statistical analysis to indicate the precision of an estimate (for example, estimate of effect size). Credible intervals ( $\mathrm{CrI})$ in Bayesian statistics could be considered as analogous to confidence interval (CI) in non-Bayesian (or frequentist) statistical analysis, reflecting the precision of an estimate. A 95\% credible interval can be interpreted as the following: there is $95 \%$ probability that the true treatment effect lies in a 95\% credible interval., ${ }^{2,3}$

\section{Model interpretation}

Defining $r_{i k}$ as the number of events (occurrence of CI-AKI), out of the total number of patients in each arm, $n_{i k}$, for arm $k$ of trial $i$, we assume that the data generation process follows a Binomial likelihood i.e.

$$
r_{i k} \sim \operatorname{Binomial}\left(p_{i k}, n_{i k}\right)
$$

where $p_{i k}$ represents the probability of an event in arm $k$ of trial $i(i=1,2 \ldots 139$; $k=1,2,3,4) \cdot p_{i k}$ can only take values between 0 and 1 . We model the probabilities of 
events $p_{i k}$ on the logit scale as

$$
\operatorname{logit}\left(p_{i k}\right)=\mu_{i}+\delta_{i, j k} I_{\{k \neq 1\}}
$$

where

$$
\begin{aligned}
& I\{u\}=1 \text { if } u \text { is true } \\
& I_{\{u\}}=0 \text { otherwise }
\end{aligned}
$$

In this setup, $\mu_{i}$ are trial-specific baselines, representing the log-odds of the outcome in the 'control' treatment, $\delta_{i, j k}$ are the trial-specific log-odds ratios of events on the treatment group $k$ compared to $j$.

\section{Parameterization of the model:}

The probabilities of event in the arms of a study $p_{i k}$ can be parameterized in terms of the log-odds ratios (OR). The underlying trial-specific effect are defined as $\theta_{i, j k}$; the $\log (O R)$ of treatment $k$ relative to $j$ in study $i$.

\section{Random effects model:}

For a random effects model the trial-specific log-odds ratios come from a common distribution:

$$
\delta_{i, j k} \sim N\left(d_{j k,} \sigma^{2}\right)
$$

where $d_{j k}$ is the multiple-treatments meta-analysis estimate of the relative effect of treatment $j$ relative to $k$ and $\sigma$ is the heterogeneity standard deviation assumed common across comparisons.

\section{Fixed effect model:}

For a fixed effect model we replace equation (1) with

$$
\operatorname{logit}\left(p_{i k}\right)=\mu_{i}+d_{i k} I_{\{k \neq 1\}}
$$


which is equivalent to setting the between-trial heterogeneity $\sigma^{2}$ to zero thus assuming homogeneity of the underlying true treatment effects.

\section{Consistency model:}

Assuming consistency, the means of the random effects distribution are related. Selecting $T-1$ basic parameters $\mu_{A k}$, all means are related via $\mu_{j k}=\mu_{A k}-\mu_{A j \text {. }}$

\section{Inconsistency model:}

In a random effects inconsistency model, no association between the $\mu_{A k} \mathrm{~s}$ are assumed, so the model is a series of independent comparison-specific meta-analyses which however share the same heterogeneity parameter $\sigma^{2}$.

In a fixed effects inconsistency model no shared variance parameter needs to be considered. The inconsistency model is then equivalent to performing completely separate pairwise meta-analysis of the data.

\section{Meta-regression and subgroup model:}

The model specification considered is to assume that all treatment by covariate interactions (for all treatments vs the common control comparator) are identical; that is, the same regression coefficient $(\beta)$ is assumed regardless of treatment (excluding control) implying the same covariate effect for each treatment relative to control. A prior distribution is given for the common regression coefficient.

$$
\delta_{j b k} \sim \begin{cases}\operatorname{Normal}\left(d_{A k}+\beta X_{j}, \sigma^{2}\right) \sim \operatorname{Normal}\left(d_{A k}-d_{A A}+\beta X_{j}, \sigma^{2}\right) & \text { if } b=A \\ \operatorname{Normal}\left(d_{b k}, \sigma^{2}\right) \sim \operatorname{Normal}\left(d_{A k}-d_{A b}, \sigma^{2}\right) & \text { if } b \neq A\end{cases}
$$




$$
\begin{aligned}
r_{j k} & \sim \operatorname{Binomial}\left(p_{j k}, n_{j k}\right) \quad \text { for trial } j, \text { treatment } k \\
\operatorname{logit}\left(p_{j k}\right) & =\left\{\begin{array}{cc}
\mu_{j b} & b=A, B, C, \ldots \quad \text { if } k=b \\
\mu_{j b}+\delta_{j k b} & \text { if } k \text { alphabetically after } b
\end{array}\right.
\end{aligned}
$$

$\mu_{j b}$ is the log odds of an event in trial $j$ on 'baseline' treatment $b, \delta_{j b k}$ is the trial-specific $\log$ odds ratio of treatment $k$ relative to treatment $b$ in trial $j$. The pooled log odds ratios, $d_{b k}$, are identified by expressing them in terms of the reference treatment $A, d_{A k}-d_{A b}$, where $d_{A A}$ is set equal to zero. The between-study variance $\sigma^{2}$ is assumed constant for all treatment comparisons. ${ }^{4}$

\section{SUCRA}

The treatments can be ranked according to their effectiveness. The order of treatment in every MCMC circle is calculated as

$$
\operatorname{order}_{k}=\sum_{j=1}^{n t} I\left(d_{j} \leq d_{k}\right)
$$

where $I$ ( $\left.d_{j} \leq d_{k}\right)=1$ if $d_{j} \leq d_{k}$ and 0 otherwise. The probability of treatment $k$ to be at the $j$ order is estimated from the quantity effectiveness $k, j$ and the cumulative probabilities by cum.effectiveness $k$.j. Then the surface under the cumulative ranking curve (SUCRA) for the treatment is

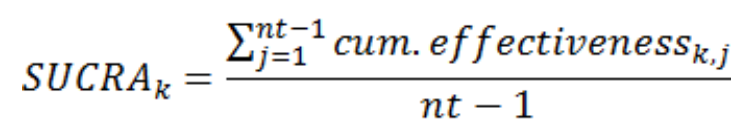

\section{Model fit}

We checked whether a model's fit is satisfactory using the deviance information criterion (DIC). DIC is the sum of Dbar (the posterior mean residual deviance) and the leverage, $P d$ (also termed the effective number of parameters). The model fits the data 
adequately when Dbar is approximative with the number of data points. $P d$ provides a measure of model complexity. Then the DIC means a measure of model fit that penalizes model complexity - lower values of the DIC suggest a more parsimonious model.

In order to assess whether the model provided adequate fit, we calculated DICs of four models, including random consistency, random inconsistency, fixed consistency, fixed inconsistency model within a Bayesian framework using the WinBUGS and R software.

\section{Assessment of inconsistency}

A "direct" estimate of the C vs. B effect, $\hat{}_{B C}^{\text {dir }}$, is to be compared to an "indirect" estimate, $\hat{d}_{\mathrm{B} C}^{\text {ind }}$, formed from the $\mathrm{AB}$ and AC direct evidence

$$
\begin{aligned}
& \hat{d}_{B C}^{i n d}=\hat{d}^{d i r}-\hat{d}_{A B}^{d i r}
\end{aligned}
$$

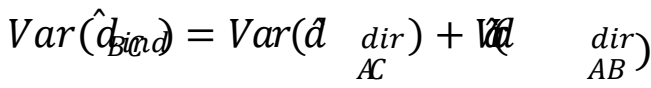

We assume that the direct estimates can either be estimates from individual trials. An estimate of the inconsistency, $\omega$, can be formed by simply subtracting the direct and indirect estimates:

$$
\begin{gathered}
\hat{A}_{B C}=\hat{\omega}_{B C}^{d i r}-\hat{d}_{B C}^{\text {ind }} \\
\operatorname{Var}\left(\hat{\omega}_{B C}\right)=\operatorname{Var}\left(\hat{d}_{B C}^{\text {dir }}\right)+\operatorname{Var}\left(\hat{d}_{B C}^{i n d}\right)=\operatorname{Var}\left(\hat{d}_{B C}^{d i r}\right)+\operatorname{Var}\left(\hat{d}_{A B}^{d i r}\right)+\operatorname{Var}\left(\hat{d}_{A C}^{d i r}\right)
\end{gathered}
$$

An approximate test of the null hypothesis that there is no inconsistency can be obtained by referring $Z_{B C}=\frac{\widehat{\omega}_{B C}}{\sqrt{\operatorname{Var}\left(\widehat{\omega}_{B C}\right)}}$ to the standard normal distribution. the method can only be applied to 3 independent sources of data. Obviously, the method can only be applied to 3 independent sources of data. This idea can be extended to all loops formed in the 
network and plot the $\omega$ together with its 95\% confidence interval. In the presence of consistency within a loop all intervals should be compatible with zero.

Another way to infer about consistency in the network as a whole is to compare the DICs between the consistency and inconsistency model. If the DIC assuming inconsistency is lower than the DIC assuming consistency by three or more units, then the assumption of consistency is likely to be violated.

\section{Reference:}

1. Schmidt FL, Hunter JE. Development of a general solution to the problem of validity generalization. J Appl Psychol. 1977;62(5):529-540.

2. Whitener EM. Confusion of confidence intervals and credibility intervals in metaanalysis. J Appl Psychol. 1990;75(3):315-321.

3. Caird JK, Willness CR, Steel P, Scialfa C. A meta-analysis of the effects of cell phones on driver performance. Accid Anal Prev.2008;40(4):1282-1293.

4. Cooper NJ, Sutton AJ, Morris D, Ades AE, Welton NJ. Addressing between-study heterogeneity and inconsistency in mixed treatment comparisons: Application to stroke prevention treatments in individuals with non-rheumatic atrial fibrillation. Stat Med. 2009;28(14):1861-1881 